Chair for Management Sciences and Energy Economics University of Duisburg-Essen

EWL Working Paper No. 05/2011

\title{
Mean-Variance Optimization of Power Generation Portfolios under UnCERTAinty in the Merit ORDER
}

by

Malte Sunderkötter

and

Christoph Weber 


\title{
Mean-Variance optimization of power generation portfolios under uncertainty in the merit order
}

\author{
by Malte Sunderkötter and Christoph Weber
}

\begin{abstract}
In this article we discuss welfare-optimal capacity allocation of different electricity generation technologies available for serving system demand. While the classical peak load pricing theory derives the efficient portfolio structure from a deterministic marginal production cost curve ("merit order"), we investigate in particular the implications of possible reversals in the merit ordersometimes also referred to as fuel switch risks - induced by uncertain operating costs.

We propose a static, non-convex optimization model combining the classic peak load pricing model with elements of mean-variance portfolio (MVP) theory and analytically discuss possible solution cases and important optimality properties. We examine the approach in a case study on the efficient structure of generation portfolios consisting of CCGT and hard coal technologies in Germany. With special emphasis, we study the emergence of overcapacities (exceeding maximal demand) in efficient portfolios and show that diversification is not beneficial per-se. The results show that the efficient technology mix may be significantly impacted by a risk for reversals in the merit order. Therefore, our findings support the importance of considering this risk factor especially with long-term investment horizons.

The model is applicable to various investment problems related to production of nonstorable goods under price uncertainty of input factors. Similar problems can e.g. be found in transportation systems or in the process industry.

Keywords : power plant investments, peak load pricing, mean-variance portfolio theory, fuel mix diversification JEL-Classification : G11, L94, Q43, C44

DIPL. WI.-ING. MALTE SUNDERKÖTTER

Chair for Management Sciences and Energy Economics, University of Duisburg-Essen (Campus Essen)

Universitätsstr. 11, 45117 Essen

++49 - (0)69 / 9150-2192

www.ewl.wiwi.uni-due.de

malte.sunderkoetter@stud.uni-due.de

PROF. DR. CRISTOPH WEBER

Chair for Management Sciences and Energy Economics, University of Duisburg-Essen (Campus Essen) Universitätsstr. 11, 45117 Essen ++49 - (0)201 / 183-2966 www.ewl.wiwi.uni-due.de christoph.weber@uni-due.de
\end{abstract}

The authors are solely responsible for the contents which do not necessarily represent the opinion of the Chair for Management Sciences and Energy Economi 


\section{Introduction}

In the next decades, the European power industry will face an immense need for investments to renew and extend its power plant fleet. The challenge is massively increased by the required transformation to reach emission reduction targets and reduce the carbon-intensity of the power system: For a 25\% reduction in greenhouse-gas emissions by 2020 compared with 1990, the IEA (2010) estimates required generation capacity additions in European OECD countries of 337GW between 2010 and 2020 and another 498GW between 2021 and 2035. The more ambitious reduction target of $80 \%$ by 2050 (respectively $40 \%$ by 2020) as agreed by the European representants during the G8 meeting in L'Aquila in July 2009 would require consequently a much more drastic change of the generation system. To reach the reduction targets, the EU and national states have implemented several measures and development schemes which aim to politically influence investment decisions in new generation capacities directly or through monetary incentives.

For quantifying the costs and effects of these measures and subsidies on the long-term optimal system fuel mix from a welfare perspective, it is not only crucial to valuate expected total life-cycle costs, but also the economic risks conveyed with investment and operation of the plant fleet. With fuel prices fluctuating considerably, cost volatility becomes a severe risk that influences the investment economics and therewith the optimal fuel mix within the system. Thereby, short-term fuel price shocks as well as by longer-term structural changes in the commodity markets can even lead to changes in the merit order of the generation technologies. Under these circumstances, it is not possible to derive an unambiguous merit order as assumed in the classic peak-load pricing problem. In the analytical literature on generation investments, the effect of reversals in the merit order through fuel price fluctuations is commonly neglected and restricted assumptions are made to ensure an unambiguous merit order based on marginal costs of production. Although simulation-based investment optimization approaches (as e.g. discussed in Weber, 2005; Fleten et al., 2007) may capture this type of uncertainty, analytical solutions of peak-load pricing based frameworks limit fuel price risk-if captured at all - to the extent that unambiguity of the merit order is still satisfied (cf. Sunderkötter and Weber, 2009). However, long-term investment decisions such as for power plants with lifetimes of several decades may be heavily impacted by possible reversals in the order marginal costs.

The optimization problem and the solutions proposed in this paper can be easily transferred to investment decisions in several other industries besides power generation where similar problem characteristics can be found. Thereby, three main properties characterize the considered type of investment problems: (a) Availability of alternative production technologies, (b) non-storability of produced good, and (c) price uncertainty of input factors. Potential areas of application in other industries are

- Transportation systems: Vehicle fleet operators as e.g. taxi companies or logistics providers face a trade-off between investment costs and operating costs of different engine types when deciding on new investments. Thereby, the investment decision is typically subject to an expected or pre- 
scheduled annual transportation performance. While retail prices for diesel fuels were traditionally lower compared to gasoline in past decades, the picture changed in many European countries in 2008 due to changes in demand and refinery capacities (Eurostat, 2009). In the U.S., the order of retail diesel and gasoline fuel prices changed several times between 2007 and 2009 (EIA, 2009). Moreover, the expected further emergence of hybrid and electric technologies may lead to significant changes in the order of operating costs among all vehicle technologies.

- Process industry: Many industrial production processes, e.g. in chemical industry, show similar trade-offs between investment costs and uncertain costs of required input factors. Replacement of a certain chemical in case of price shocks is usually only a mid-term option, since process changes usually cause investment costs. $^{2}$

This paper is structured as follows: After a brief recall of the deterministic peak load pricing concept in Section 2.1, the risk-extended optimization problem is proposed in Section 2.2. The analytical solution and its properties are discussed starting in Section 2.3 for the simplified case without reversal risk, before the general portfolio problem is treated. Thereby, the two special cases of purely cost-efficient and purely risk-efficient portfolios are elucidated in Sections 2.4 and 2.5, before the combined problem is discussed in Section 2.6. Section 3 treats the determination of the probability for reversals in the merit order. This issue is first discussed for the reversal risk within one period, before the considerations are augmented for the reversal risk over a plant's lifetime. In Section 4, we examine the model in a case study on the cost-risk efficient structure of generation portfolios consisting of CCGT and hard coal technologies in Germany. The paper concludes in Section 5 with a summary and critical acclaim of our key findings.

\section{Modelling optimal investment policies in electricity markets given uncertainty in the merit order}

\subsection{Deterministic peak-load pricing problem}

Our investment model for the electricity system is based on a two-stage decision problem following the classic peak load pricing literature (see e.g. Crew et al., 1995): On the first stage, the decision to invest in a portfolio of different available plant technologies $u \in\{1, \ldots, n\}$ with capacity $K_{u}$ is made. At this point in time, investors are assumed to have full information about investment costs $C_{i n v, u}$ of each technology and

\footnotetext{
${ }^{2}$ Recently, severe delivery shortages of a chemical mass product caught particular attention (cf. e.g. Bonilla, 2010): Acetonitrile, a by-product of acrylnitrile which is widely used in the plastics and textile industry, is a frequently used solvent in laboratories and industrial processes. Due to the world-wide demand drop of plastic products combined with temporarily decommissioned production capacities, the price of acetonitrile exploded in 2008/2009 from few euro per liter up to a hundred. The "Great Acetonitrile Shortage", as it has come to be known in the industry, forces chemists to reduce the required solvent consumption or switch to other solvents. However, these alternatives are usually conveyed with additional investment costs.
} 
about the distribution of the uncertain fuel prices $\tilde{c}_{u}$. However, actual fuel price realizations are not revealed until the second stage. Then, the optimal deployment decision of each plant within the portfolio selected on the first stage is made for the total planning period $[0 ; T]$ (e.g. a year), which we assume to be broken down into time steps of equal length $t \in[0 ; T]$ (e.g. hours). Price inelastic system demand is deterministically given by the demand function $D:[0 ; T] \rightarrow \mathbb{R}_{+}, t \mapsto D(t)$, which is assumed to be continuous and (at least) two-times differentiable.

The objective is to minimize the total system cost of electricity production, $C$, consisting of total operating costs, $C_{o p, u}$, plus annualized capacity investment costs, $C_{i n v, u}$, summed over the available technologies $u$. In fact, operating costs at time $t$ are a function $C_{o p, u}\left(y_{u, t}\right)=y_{u, t} \cdot c_{u, t}$ of the instantaneous output level $y_{u, t}(\mathrm{MW})$ times the specific operating $\operatorname{costs} c_{u, t}(€ / \mathrm{MWh})$. Furthermore, total investment costs are determined by the installed capacity $K_{u}$ and the specific investment costs $c_{i n v, u}\left(€ / \mathrm{MW}_{e l}\right)$ and can be expressed by $C_{i n v, u}\left(K_{u}\right)=K_{u} \cdot c_{i n v, u}$. Therefore, the plant capacities $K_{u}$ and the corresponding output levels $y_{u, t}$ are the decision variables to be optimized. Taking into account that demand must never exceed available capacities, the deterministic optimization problem may be written as

$$
\begin{aligned}
C^{*}= & \min _{y_{u, t}, K_{u}} C\left(y_{u, t}, K_{u}\right) \\
\text { s.t. } C= & \int_{0}^{T} \sum_{u} y_{u, t} \cdot c_{u, t} \mathrm{~d} t+\sum_{u} K_{u} \cdot c_{i n v, u} \\
& y_{u, t}-K_{u} \leq 0 \quad \forall t, u \\
& \sum_{u} y_{u, t} \geq D(t) \quad \forall t
\end{aligned}
$$

In the following paragraph, we well extend this problem to reflect not only the expected costs but also cost risks in the optimization.

\subsection{The risk-adjusted portfolio problem}

In classic portfolio theory, investors are assumed to select efficient portfolios solely based on the expected return and risk (in the form of variance of return) of the available assets. We adopt this decision principle also for the cost based investment decision of generation assets in the system portfolio. Following Jansen et al. (2006) and Gotham et al. (2009), we thus use total system costs instead of "return" and variance of total costs as the relevant risk measure.

As frequently used in investment literature, we assume that society's preferences are represented by a function of the form

$$
L=\mathbf{E}[C]+\frac{1}{2} A \cdot \operatorname{Var}[C]
$$

where $A$ denotes the investors' risk attitude (see e.g. Trautmann, 2006; Sunderkötter and Weber, 2009). For normally distributed payoffs, this preference function is induced by exponential utilities with constant 
absolute risk aversion and shows in maximization problems consistency with the rationale of expected utility maximization.

To allow for a better traceability of the solution, we confine in the following to the case with only two technologies, i.e. $n=2 .{ }^{3}$ Given uncertain fuel prices which lead to fluctuating operating costs $\tilde{c}_{1}$ and $\tilde{c}_{2}$, two possible scenarios are to be distinguished on the second stage for the merit order. These states of the merit order are indicated by the state variable $\tilde{s}$ with realizations $s_{i}, i \in\{1 ; 2\}$ defined as

$$
\tilde{s}:= \begin{cases}s_{0}, & \text { for } c_{1} \leq c_{2}(\text { "default order") } \\ s_{1}, & \text { for } c_{1}>c_{2}(\text { "reverse order") }\end{cases}
$$

Let periodic operating $\operatorname{costs} \tilde{c}_{u}, u \in\{1 ; 2\}$ be represented by bivariate jointly distributed random variables with joint probability density function $\varphi_{1,2}\left(c_{1}, c_{2}, \rho\right)$ and mean $\bar{c}_{u} \cdot{ }^{4}$ For each technology $u, \tilde{c}_{u}$ is a (univariately) distributed random variable on the probability space $(\Omega, \mathcal{A}(\mathbb{R}), \mathbf{P})$ with $\tilde{c}_{u}: \Omega \rightarrow \mathbb{R}$ with marginal probability density function $\varphi_{u}\left(c_{u}\right)$ and marginal cumulative distribution $\Phi_{u}\left(c_{u}\right)$ and corresponding mean $\bar{c}_{u}$, variance $\sigma_{u}^{2}$, and correlation $\rho$. Then, $\tilde{s}\left(\tilde{c}_{1}, \tilde{c}_{2}\right)$ is itself a (discrete) random variable generated by $\tilde{c}_{1}, \tilde{c}_{2}$ on the probability space $\left(\Omega^{\prime}, \mathcal{B}\left(\mathbb{R}^{2}\right), \mathbf{P}^{\prime}\right)$, with $\tilde{s}: \Omega^{\prime} \rightarrow\left\{s_{0} ; s_{1}\right\}, \Omega^{\prime}=\Omega_{1} \times \Omega_{2}$.

The likelihood for a reversal in the merit order is denoted by $\mathbf{P}\left(\tilde{s}=s_{1}\right):=\mathbf{P}\left(c_{1}>c_{2}\right)$. By definition, $\mathbf{P}\left(\tilde{s}=s_{0}\right):=\mathbf{P}\left(c_{1} \leq c_{2}\right)=1-\mathbf{P}\left(\tilde{s}=s_{1}\right)$ is thus the probability that no reversals of annual operating costs in the merit order occur. Intuitively, the reversal risk depends on the assumed time series model for the operating costs $c_{1}$ and $c_{2}$. We will come back to the computation of the reversal risk in Section 3 . Taking into account unambiguity of the plant merit order, the classic peak load pricing framework to model total system costs has to be extended. Considering two generation technologies with the resulting possible fuel price orders according to definition (6), the following states of the plant output variable $y_{t}$ have to be distinguished:

$$
\begin{aligned}
& \tilde{y}_{1, t}= \begin{cases}D(t), & \text { for } \tilde{s}=s_{0} \wedge D(t)<K_{1} \\
K_{1}, & \text { for } \tilde{s}=s_{0} \wedge D(t) \geq K_{1} \\
0, & \text { for } \tilde{s}=s_{1} \wedge D(t)<K_{2} \\
D(t)-K_{2}, & \text { for } \tilde{s}=s_{1} \wedge D(t) \geq K_{2}\end{cases} \\
& \tilde{y}_{2, t}= \begin{cases}0, & \text { for } \tilde{s}=s_{0} \wedge D(t)<K_{1} \\
D(t)-K_{1}, & \text { for } \tilde{s}=s_{0} \wedge D(t) \geq K_{1} \\
D(t), & \text { for } \tilde{s}=s_{1} \wedge D(t)<K_{2} \\
K_{2}, & \text { for } \tilde{s}=s_{1} \wedge D(t) \geq K_{2}\end{cases}
\end{aligned}
$$

\footnotetext{
${ }^{3}$ The solution to the extended portfolio problem with $n$ technologies given the case of a deterministic merit order is discussed in Sunderkötter and Weber (2009).

${ }^{4}$ Throughout this article, random variables are indicated by a “ ”, whereas their realizations are written as plain letters.
} 
We assume without limitation of generality $D(t)$ to represent demand in a decreasing order and thus to be strictly monotone decreasing in $t$ with $D(0)=D_{\max }$. This allows to simplify problem (1)-(4) by using the minimum ${ }^{5}$ operating duration $O_{u}$ as decision variables instead of $y_{u, t}$.

Given the strict monotony of the load duration curve, there is a unique mapping between capacities and operating times for each merit order state. Obviously, the upper bound of the optimal operating time of technology 1 equals $T$ in case of the default order where technology 1 represents the base load technology. By defining $R(K)$ as the inverse of the demand function $D(t)$, the minimum operating duration of the base technology $1 \mathrm{in}$ the default merit order is determined by $O_{1}=t_{1}=R\left(K_{1}\right)$. Equivalently, $t_{1}$ determines the upper bound of the operating time of the respective peak technology. For the latter, the minimum operating duration equals zero. The minimum operating duration of technology one is reduced to a value $O_{1}=0$ in the reverse case in favor of technology 2, which then becomes the new base load technology running at least during $\mathrm{O}_{2}=t_{u}$. With possible reversals in the merit order the lower bound of the ex-ante optimal operation time of technology 1 reduces with increasing probability for reversals. Therefore, both $t_{1}^{*}$ (lower bound of the optimal operating time of technology 1 given the default order) and $t_{2}^{*}$ (lower bound of the optimal operating time of technology 2 given the reverse order) have to be determined endogenously in the optimization problem. Recapitulating the optimal plant dispatch, the minimum operating times $O_{1}, O_{2}$ of technology 1 and 2 over the two considered merit order states can be formulated as:

$$
O_{1}=\left\{\begin{array}{ll}
t_{1} & \text { for } \tilde{s}=s_{0} \\
0 & \text { for } \tilde{s}=s_{1}
\end{array} \quad O_{2}= \begin{cases}0 & \text { for } \tilde{s}=s_{0} \\
t_{2} & \text { for } \tilde{s}=s_{1}\end{cases}\right.
$$

Note that through the invertible function $D(t)$, we can use $t_{u}$ and $K_{u}$ interchangeably as decision variables. Finally, we introduce for the operating costs of technology $u$ the conditional expectation $\mathbf{E}\left[\tilde{c}_{u} \mid \tilde{s}\right] \equiv \bar{c}_{u \mid \tilde{s}}$ and the conditional variance $\operatorname{Var}\left[\tilde{c}_{u} \mid \tilde{s}\right] \equiv \sigma_{u \mid \tilde{s}}^{2}$. The computation of these conditional parameters from a bivariate density $\varphi_{1,2}\left(c_{1}, c_{2}\right)$ is derived in Appendix B.1.

Based on these pre-considerations, the extended portfolio optimization problem with uncertain merit order can now be rewritten taking into account that the total variance is the sum of inter-scenario and

\footnotetext{
${ }^{5}$ Analogously, the problem could be formulated using maximum operating durations as decision variables.
} 

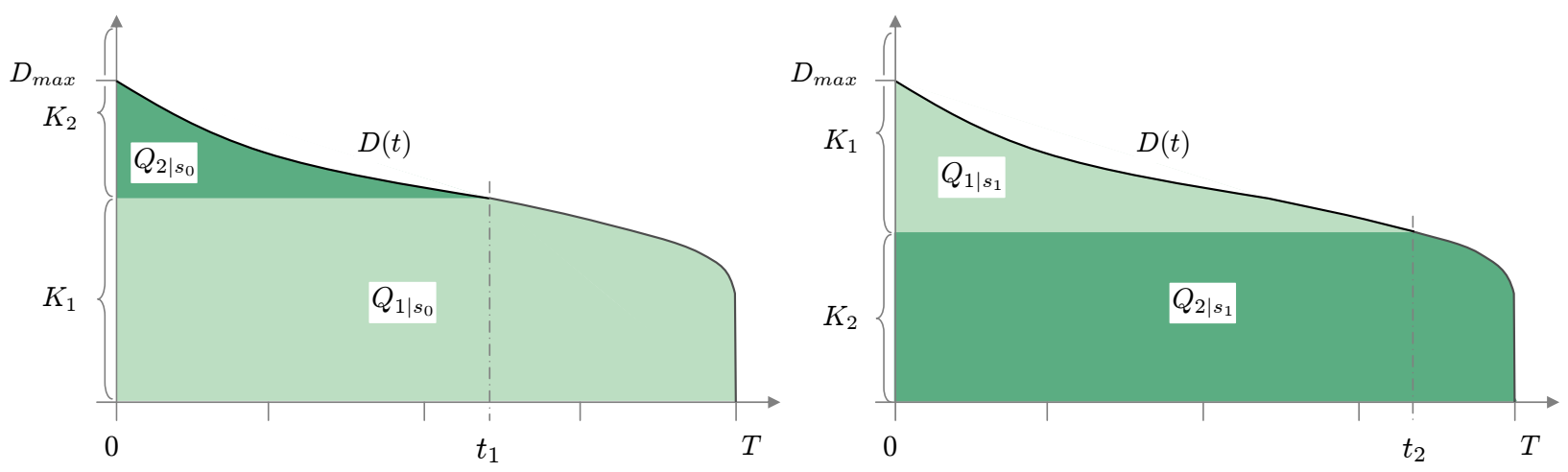

Figure 1: Reversals in the merit order influence the produced energy of technologies 1 and 2 in the default (left) and reverse case (right). Note that total installed capacity may exceed the maximal demand for $\lambda=0$.

intra-scenario variance as shown in Appendix B.2: ${ }^{6}$

$$
\begin{aligned}
L^{*} & =\min _{K_{1}, K_{2}} L \\
\text { s.t. } & L=\sum_{u=1}^{2}\left(K_{u} c_{i n v, u}+\mathbf{E}\left[Q_{u \mid \tilde{s}} \mathbf{E}\left[\tilde{c}_{u} \mid \tilde{s}\right]+\frac{A}{2} Q_{u \mid \tilde{s}}^{2}\left(\operatorname{Var}\left[\tilde{c}_{u} \mid \tilde{s}\right]+\mathbf{E}\left[\tilde{c}_{u} \mid \tilde{s}\right]^{2}\right)\right]-\frac{A}{2} \mathbf{E}\left[Q_{u \mid \tilde{s}} \mathbf{E}\left[\tilde{c}_{u} \mid \tilde{s}\right]\right]^{2}\right) \\
& +A \mathbf{E}\left[Q_{1 \mid \tilde{s}} Q_{2 \mid \tilde{s}}\left(\operatorname{Cov}\left[\tilde{c}_{1}, \tilde{c}_{2} \mid \tilde{s}\right]+\mathbf{E}\left[\tilde{c}_{1} \mid \tilde{s}\right] \cdot \mathbf{E}\left[\tilde{c}_{2} \mid \tilde{s}\right]\right)\right]-A \mathbf{E}\left[Q_{1 \mid \tilde{s}} \mathbf{E}\left[\tilde{c}_{1} \mid \tilde{s}\right]\right] \cdot \mathbf{E}\left[Q_{2 \mid \tilde{s}} \mathbf{E}\left[\tilde{c}_{2} \mid \tilde{s}\right]\right] \\
& D(0)-K_{1}-K_{2} \leq 0 \\
& K_{1}, K_{2} \geq 0 .
\end{aligned}
$$

As visualized in Figure 1, $Q_{u \mid s_{i}}$ denotes the energy produced by plant technology $u$ over the planning period $[0, T]$ given the fuel price state $s_{i}$ with

$$
\begin{aligned}
Q_{1 \mid s_{0}} & =\int_{0}^{K_{1}} R(\kappa) \mathrm{d} \kappa & Q_{2 \mid s_{0}} & =\int_{K_{1}}^{D(0)} R(\kappa) \mathrm{d} \kappa=Q_{E}-Q_{1 \mid s_{0}} \\
Q_{2 \mid s_{1}} & =\int_{0}^{K_{2}} R(\kappa) \mathrm{d} \kappa & Q_{1 \mid s_{1}} & =\int_{K_{2}}^{D(0)} R(\kappa) \mathrm{d} \kappa=Q_{E}-Q_{2 \mid s_{1}} .
\end{aligned}
$$

Here, $Q_{E}:=\int_{0}^{T} D(t) \mathrm{d} t=\int_{0}^{D(0)} R(\kappa) \mathrm{d} \kappa$ denotes the total energy demand in $[0, T]$.

\footnotetext{
${ }^{6}$ Notably, the objective function is different from the plain expected value of the preference function over the two fuel price order scenarios which would be $\mathbf{E}\left[\mathbf{E}[C \mid \tilde{s}]+\frac{1}{2} A \cdot \operatorname{Var}[C \mid \tilde{s}]\right] \neq L$.
} 
2.3. Standard solutions to the portfolio problem in the two-technology case with a deterministic merit order

Before approaching the unrestricted problem, we will first discuss possible solution cases assuming a deterministic merit order without reversal risk, i.e. $\mathbf{P}\left(\tilde{s}=s_{1}\right)=0$. Then, problem (9)-(12) reduces to

$$
\begin{array}{ll}
L_{0}^{*}=\min _{K_{1}, K_{2}} L_{0} \\
\text { s.t. } & L_{0}=\sum_{u=1}^{2} c_{i n v, u} K_{u}+c_{u} Q_{u}+\frac{A}{2}\left(\sum_{u=1}^{2} \sum_{v=1}^{2} \sigma_{u v} Q_{u} Q_{v}\right) \\
& D(0)-K_{1}-K_{2} \leq 0 \\
& K_{1}, K_{2} \geq 0
\end{array}
$$

As shown in Sunderkötter and Weber (2009), the central optimality condition can be obtained from the KKT-conditions as

$$
A\left(\left(\sigma_{1}^{2}-\sigma_{12}\right) Q_{E}-\left(\sigma_{1}^{2}+\sigma_{2}^{2}-2 \sigma_{12}\right) Q_{2}\right)=\frac{c_{i n v, 2}-c_{i n v, 1}}{t_{1}}-\bar{c}_{1}+\bar{c}_{2}
$$

with

$$
Q_{2}=\int_{0}^{t_{1}} D(t)-D\left(t_{1}\right) \mathrm{d} t, \quad Q_{E}=\int_{0}^{T} D(t) \mathrm{d} t=Q_{1}+Q_{2} .
$$

In Eqn. (19), the risk term and the cost term are separated, each to one side of the equation. Although an explicit formulation of the optimal operating time (and therewith capacities) is not possible for a generic load duration function $D(t)$, the optimality condition allows to draw conclusions on the structure of efficient portfolios. For that, the risk term on the left side and the cost term on the right side of Eqn. (19), respectively, are denoted by

$$
\begin{aligned}
& l_{0}\left(t_{1}\right):=A\left(\left(\sigma_{1}^{2}-\sigma_{12}\right) Q_{E}-\left(\sigma_{1}^{2}+\sigma_{2}^{2}-2 \sigma_{12}\right) Q_{2}\right), \\
& r_{0}\left(t_{1}\right):=\frac{c_{i n v, 2}-c_{i n v, 1}}{t_{1}}-\bar{c}_{1}+\bar{c}_{2} .
\end{aligned}
$$

Thereby, the intersection of $l_{0}\left(t_{1}\right)$ and $r_{0}\left(t_{1}\right)$ characterizes a stationary point $t_{1}^{o}$ which is necessary for the solution of the cost-variance efficient portfolio. Analysis of $l_{0}\left(t_{1}\right)$ and $r_{0}\left(t_{1}\right)$ shows that three characteristic portfolio structures can be distinguished for the purely cost-efficient and the variance efficient portfolio with either technology 1, technology 2, or both technologies being part of the efficient portfolio. For the cost efficient portfolio with $A=0$, three different solution cases can be derived from the condition $r_{0}\left(t_{1}\right)=0$ :

Property 2.1. The purely cost efficient portfolio with $A=0$ consists of

$\begin{array}{lcr}\text { Only technology } 1 & \text { for } & c_{i n v, 1}-c_{i n v, 2} \leq 0, \\ \text { Technology } 1 \text { and } 2 & \text { for } & 0<\frac{c_{i n v, 1}-c_{i n v, 2}}{\bar{c}_{2}-\bar{c}_{1}}<T, \\ \text { Only technology } 2 & \text { for } & \frac{c_{i n v, 1}-c_{i n v, 2}}{\bar{c}_{2}-\bar{c}_{1}} \geq T .\end{array}$


Similarly, three solution cases can be stated for the variance minimal portfolio with $A \rightarrow \infty$ :

Property 2.2. The purely variance efficient portfolio with $A \rightarrow \infty$ consists of

$\begin{array}{lll}\text { Only technology } 1 & \text { for } & \sigma_{1}^{2} \leq \sigma_{12}, \\ \text { Technology } 1 \text { and } 2 & \text { for } & \left(\sigma_{1}^{2}>\sigma_{12}\right) \wedge\left(\sigma_{2}^{2}>\sigma_{12}\right), \\ \text { Only technology } 2 & \text { for } & \sigma_{2}^{2} \leq \sigma_{12} .\end{array}$

As a consequence of Properties 2.1 and 2.2, stationary points in nine different cases can be distinguished to determine the general set of solutions for combined cost-variance efficient portfolios as shown in Figure 2. Since the optimization problem is only convex in the case with both technologies being part of the cost-minimal and the variance-minimal portfolios (case (V), Figure 2) as shown in Sunderkötter and Weber (2009), first derivatives can be used as sufficient test for a local minimum in the other cases: Thereby, according to the mean value theorem, a stationary point $t_{1}^{o}$ is a minimum to $\mathcal{L}_{0}$ only if there exists a $r \in \mathbb{R}_{+}$such that for all $t_{1} \in\left(t_{1}^{o}-r, t_{1}^{o}\right]$ it is $\frac{\partial \mathcal{L}_{0}}{\partial K_{1}}=l_{0}\left(t_{1}\right)-r_{0}\left(t_{1}\right) \leq 0$, and for every $t_{1} \in\left[t_{1}^{o}, t_{1}^{o}+r\right)$ it is $\frac{\partial \mathcal{L}_{0}}{\partial K_{1}}=l_{0}\left(t_{1}\right)-r_{0}\left(t_{1}\right) \geq 0$.

The identified stationary points allow to formulate the following properties for the structure of costvariance efficient portfolios given a deterministic merit order $\left(\mathbf{P}\left(s_{1}\right)=0\right)$ :

Property 2.3. If both technologies 1 and 2 are included in the purely cost-efficient portfolio (i.e. A=0) and in the purely variance-efficient portfolio (i.e. $A \rightarrow+\infty$ ), then they are also included in all cost-variance efficient portfolios with $A>0$.

Property 2.4. If technology $u$ is neither included in the purely cost-efficient portfolio nor in the purely variance-efficient portfolio, then $u$ is not included in any cost-variance efficient portfolio with $A>0$.

Property 2.5. If the purely cost-efficient (purely variance-efficient) portfolio consists only of technology $u \in\{1,2\}$ and the purely variance-efficient (purely cost-efficient) portfolio consists of both technologies, then there exists an $A_{0}$ such that technology $u$ is included in all efficient portfolios for $A>A_{0}\left(A<A_{0}\right)$ and excluded in all efficient portfolios for $A<A_{0}\left(A>A_{0}\right)$.

\subsection{Standard solutions to the purely cost efficient portfolio with uncertainty of the merit order}

As an extreme case of the general optimization problem, we will at first consider the purely cost-efficient portfolio with an unstable merit order, i.e. the case $A=0$. Under this premise, the Lagrangian simplifies to

$$
\mathcal{L}_{c}=\sum_{u=1}^{2}\left(K_{u} c_{i n v, u}+\mathbf{E}\left[Q_{u \mid \tilde{s}} \mathbf{E}\left[\tilde{c}_{u} \mid \tilde{s}\right]\right]\right)+\lambda \cdot\left(D(0)-K_{1}-K_{2}\right)
$$




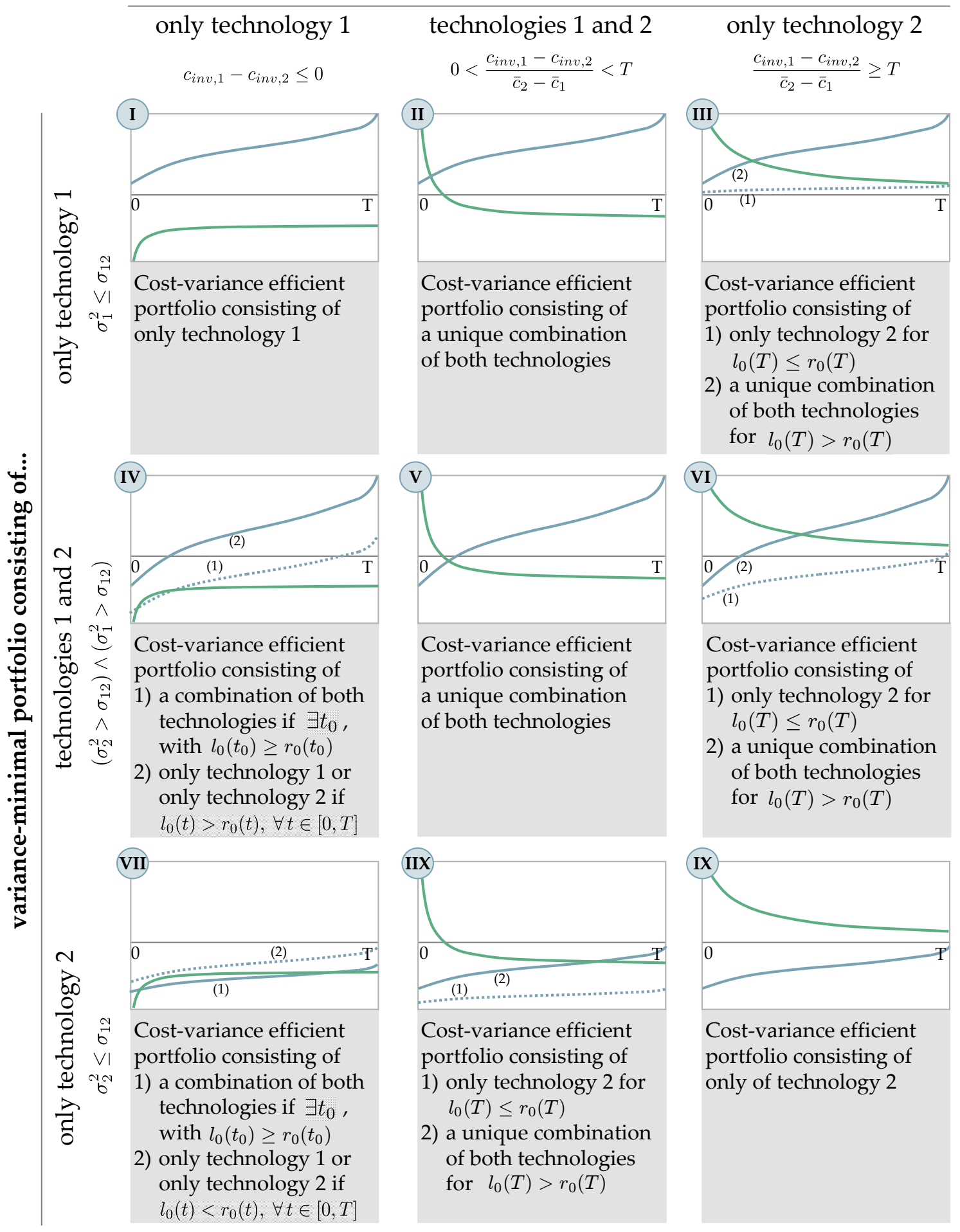

Figure 2: Solution cases for the portfolio problem without reversals in the merit order, i.e. $\mathbf{P}\left(\tilde{s}=s_{1}\right)=0$. Stationary points for cost-variance efficient portfolio combinations are characterized by the intersection of $r_{0}(t)$ (green) and $l_{0}(t)$ (blue). 
With $z$ denoting the difference in operating costs of technology 1 and 2, i.e. $z:=c_{2}-c_{1}$, the corresponding KKT-conditions can be derived as shown in Appendix B.3 as

$$
\begin{aligned}
& \frac{\partial \mathcal{L}_{c}}{\partial K_{1}}=c_{i n v, 1}-\lambda-t_{1} \mathbf{P}\left(s_{0}\right) \cdot \mathbf{E}\left[\tilde{z} \mid s_{0}\right] \geq 0 \quad \perp \quad K_{1} \geq 0 \\
& \frac{\partial \mathcal{L}_{c}}{\partial K_{2}}=c_{i n v, 2}-\lambda+t_{2} \mathbf{P}\left(s_{1}\right) \cdot \mathbf{E}\left[\tilde{z} \mid s_{1}\right] \geq 0 \quad \perp \quad K_{2} \geq 0 \\
& \frac{\partial \mathcal{L}_{c}}{\partial \lambda}=D(0)-K_{1}-K_{2} \leq 0 \quad \perp \quad \lambda \geq 0
\end{aligned}
$$

Two cases can be distinguished: For $\lambda>0$, KKT condition (31) is binding. Hence, total capacity will meet but not exceed the demand maximum in the optimum, i.e. $K_{1}+K_{2}=D(0)$. For $\lambda=0$, total installed capacity may exceed the maximal demand, i.e. $K_{1}+K_{2} \geq D(0)$. The potential "overcapacities" in this case will be economically favorable, if the probability weighted savings from operating costs in the reversed merit order case exceed the additional investment costs for the capacity surplus. The excess capacity thus increases the fleet's operating flexibility. Nevertheless, the optimal energy produced in any scenario cannot exceed total energy demand, thus it still holds

$$
Q_{E}=\int_{0}^{T} D(t) \mathrm{d} t=Q_{1 \mid s_{0}}+Q_{2 \mid s_{0}}=Q_{1 \mid s_{1}}+Q_{2 \mid s_{1}} .
$$

It is intuitively clear that in the considered model framework with ideal and deterministic plant availabilities excess capacities may only be economical if the technologies' investment costs are relatively small compared to the expected difference in operating costs. This becomes evident in view of KKT conditions (29) and (30): The shadow price $\lambda$ is linearly decreasing in $t_{1}$ and $t_{2}$, respectively, with

$$
\lambda=c_{i n v, 1}-t_{1} \mathbf{P}\left(s_{0}\right) \mathbf{E}\left[\tilde{z} \mid s_{0}\right]=c_{i n v, 2}+t_{2} \mathbf{P}\left(s_{1}\right) \mathbf{E}\left[\tilde{z} \mid s_{1}\right]
$$

Clearly, $\lambda$ is positive for $t_{1}=0, t_{2}=0$ and reaches its minimum at $t_{1}=T$ or $t_{2}=T$. Since the solution for the cost efficient portfolio with excess capacities requires $\lambda=0$, it can be concluded that this case may only exist if expected operating costs and investment costs satisfy the necessary condition

$$
\left(c_{i n v, 1}-T \mathbf{P}\left(s_{0}\right) \mathbf{E}\left[\tilde{z} \mid s_{0}\right]<0\right) \wedge\left(c_{i n v, 2}+T \mathbf{P}\left(s_{1}\right) \mathbf{E}\left[\tilde{z} \mid s_{1}\right]<0\right)
$$

Notably, condition (33) will rarely be satisfied for applications related to power generation portfolios with the cost characteristics of conventional plant technologies as shown in Section 4.

Similar to the special case with a deterministic merit order (cf. Section 2.3), three solution cases can be distinguished for purely cost efficient portfolios with uncertainty of the merit order Appendix B.3:

Proposition 2.1. With $z:=c_{2}-c_{1}$ denoting the difference in operating costs of technologies 1 and 2 , the purely cost-minimal portfolio with a risk of reversal in the merit order $\mathbf{P}\left(s_{1}\right)$ consists of technology 2 if and only if

$$
c_{i n v, 1}-c_{i n v, 2} \geq T \mathbf{P}\left(s_{0}\right) \mathbf{E}\left[\tilde{z} \mid s_{0}\right] .
$$


In contrast, the portfolio consists of technology 1 if and only if

$$
c_{i n v, 1}-c_{i n v, 2} \leq T \mathbf{P}\left(s_{1}\right) \mathbf{E}\left[\tilde{z} \mid s_{1}\right] .
$$

With other words, the purely cost efficient portfolio consists of both technologies if and only if

$$
T \mathbf{P}\left(s_{1}\right) \cdot \mathbf{E}\left[\tilde{z} \mid s_{1}\right]<c_{i n v, 1}-c_{i n v, 2}<T \mathbf{P}\left(s_{0}\right) \cdot \mathbf{E}\left[\tilde{z} \mid s_{0}\right] .
$$

\subsection{Standard solutions to the purely variance efficient portfolio with uncertainty of the merit order}

The variance efficient portfolio represents another extreme case of the general portfolio problem obtained as $A \rightarrow \infty$. The Lagrangian may be written as

$$
\begin{aligned}
\mathcal{L}_{v}=\lambda & \cdot\left(D(0)-K_{1}-K_{2}\right)+\frac{1}{2} \sum_{u=1}^{2}\left(\mathbf{E}\left[Q_{u \mid \tilde{s}}^{2} \cdot\left(\operatorname{Var}\left[\tilde{c}_{u} \mid \tilde{s}\right]+\mathbf{E}\left[\tilde{c}_{u} \mid \tilde{s}\right]^{2}\right)\right]-\mathbf{E}\left[Q_{u \mid \tilde{s}} \mathbf{E}\left[\tilde{c}_{u} \mid \tilde{s}\right]\right]^{2}\right) \\
+ & \mathbf{E}\left[Q_{1 \mid \tilde{s}} Q_{2 \mid \tilde{s}} \cdot\left(\operatorname{Cov}\left[\tilde{c}_{1}, \tilde{c}_{2} \mid \tilde{s}\right]+\mathbf{E}\left[\tilde{c}_{1} \mid \tilde{s}\right] \cdot \mathbf{E}\left[\tilde{c}_{2} \mid \tilde{s}\right]\right)\right]-\mathbf{E}\left[Q_{1 \mid \tilde{s}} \mathbf{E}\left[\tilde{c}_{1} \mid \tilde{s}\right]\right] \cdot \mathbf{E}\left[Q_{2 \mid \tilde{s}} \mathbf{E}\left[\tilde{c}_{2} \mid \tilde{s}\right]\right]
\end{aligned}
$$

The corresponding KKT-conditions can be derived as:

$$
\begin{aligned}
& \frac{\partial \mathcal{L}_{v}}{\partial K_{1}}=-\lambda-\mathbf{P}\left(s_{0}\right) t_{1} \cdot \sum_{u=1}^{2}(-1)^{u} Q_{u \mid s_{0}}\left(\sigma_{u \mid s_{0}}^{2}-\sigma_{12 \mid s_{0}}+\right. \\
& \left.+\mathbf{P}\left(s_{1}\right) \cdot\left(\bar{c}_{u \mid s_{0}}^{2}-\bar{c}_{2 \mid s_{0}} \bar{c}_{1 \mid s_{0}}-\bar{c}_{u \mid s_{0}} \sum_{v=1}^{2} \bar{c}_{v \mid s_{1}} \frac{Q_{v \mid s_{1}}}{Q_{u \mid s_{0}}}\right)\right) \geq 0, \quad \perp \quad K_{1} \geq 0 \\
& \frac{\partial \mathcal{L}_{v}}{\partial K_{2}}=-\lambda+\mathbf{P}\left(s_{1}\right) t_{2} \cdot \sum_{u=1}^{2}(-1)^{u} Q_{u \mid s_{1}}\left(\sigma_{u \mid s_{1}}^{2}-\sigma_{12 \mid s_{1}}+\right. \\
& \left.+\mathbf{P}\left(s_{0}\right) \cdot\left(\bar{c}_{u \mid s_{1}}^{2}-\bar{c}_{2 \mid s_{1}} \bar{c}_{1 \mid s_{1}}-\bar{c}_{u \mid s_{1}} \sum_{v=1}^{2} \bar{c}_{v \mid s_{0}} \frac{Q_{v \mid s_{0}}}{Q_{u \mid s_{1}}}\right)\right) \geq 0, \quad \perp \quad K_{2} \geq 0 \\
& \frac{\partial \mathcal{L}_{v}}{\partial \lambda}=D(0)-K_{1}-K_{2} \leq 0, \quad \perp \quad \lambda \geq 0
\end{aligned}
$$

Since investment costs are neglected in the purely variance optimal investment decision, it can be concluded from the existence of a variance-efficient portfolio without overcapacities that an increase in the capacity of any technology is also efficient. With other words: If additional capacity has zero cost, increasing the capacity of an efficient portfolio cannot negatively influence its optimality as long as the expected technology deployment on the second stage of the optimization problem is not changed compared to the situation without overcapacities. ${ }^{7}$ Therefore, we will restrict in the following the variance minimization problem to the more interesting case in which total installed generation capacity matches system demand. Then, the hitherto constraint inequality (11) is replaced by the equality

$$
D(0)-K_{1}-K_{2}=0
$$

\footnotetext{
${ }^{7}$ Notably, for a purely variance efficient solution the actual plant deployment on the second stage is irrelevant and could theoretically be realized arbitrarily. Only the expected plant deployment is relevant for the variance minimal investment decision on the first stage of the problem.
} 
Under this assumption it is not clear any longer whether the efficient portfolio consists of a single technology or a mix of both technologies. With (41), $t_{2}$ can be expressed as a function of the $t_{1}$ with $t_{2}\left(t_{1}\right)=$ $R\left(D(0)-D\left(t_{1}\right)\right)$. The latter expression may be used to reformulate the production volumes as defined in Eqs. (13), (14) in order to express the problem solely dependent on $t_{1}$.

For an interior solution with $K_{1}^{*}, K_{2}^{*}>0$, the KKT conditions (38) and (39) have to be satisfied with equality as necessary optimality condition. Eliminating $\lambda$ through subtraction of these conditions yields the central optimality condition $\frac{\partial \mathcal{L}_{R}}{\partial K_{1}}-\frac{\partial \mathcal{L}_{R}}{\partial K_{2}}=0$ which allows us to derive the following proposition:

Proposition 2.2. Under the restriction that total installed generation capacity must match total demand, i.e. $\lambda \neq 0$, the purely variance minimal portfolio with a risk of reversal in the merit order $\mathbf{P}\left(s_{1}\right)>0$ corresponding to optimization problem (9)-(12) consists of both technologies 1 and 2 if

$$
\left(-\frac{\sigma_{12 \mid s_{0}}-\sigma_{1 \mid s_{0}}^{2}}{\mathbf{E}\left[\tilde{z} \mid s_{0}\right] \mathbf{P}\left(s_{1}\right)}>\bar{c}_{1 \mid s_{1}}-\bar{c}_{1 \mid s_{0}}\right) \wedge\left(\bar{c}_{1 \mid s_{1}}-\bar{c}_{1 \mid s_{0}}<\frac{\sigma_{1 \mid s_{1}}^{2}-\sigma_{12 \mid s_{1}}}{\mathbf{E}\left[\tilde{z} \mid s_{1}\right] \mathbf{P}\left(s_{0}\right)}\right)
$$

The proof and supplementary discussions on other solution cases for the variance minimal portfolio are provided in Appendix B.4

\subsection{Standard solutions to the combined portfolio problem with uncertainty of the merit order}

We start solving the general non-convex problem (9)-(12) by using a standard Lagrange approach for the relaxed assumption of $\mathbf{P}\left(\tilde{s}=s_{1}\right) \geq 0$ to identify stationary points as necessary conditions in the optimum. For ease of computation, again $t_{1}, t_{2}$ are used as equivalent decision variables. Having obtained optimal values for $t_{1}^{*}, t_{2}^{*}$, we can subsequently derive $K_{1}^{*}, K_{2}^{*}$ from Eqn. (8) to complete the solution. Then, the Lagrangian $\mathcal{L}$ writes

$$
\mathcal{L}=L+\lambda \cdot\left(D(0)-K_{1}-K_{2}\right)
$$

and the corresponding KKT-conditions may be written as:

$$
\begin{array}{rlrl}
\frac{\partial \mathcal{L}}{\partial K_{1}} & =c_{i n v, 1}-\lambda-\mathbf{P}\left(s_{0}\right) t_{1} \cdot \sum_{u=1}^{2}(-1)^{u} \cdot\left(\bar{c}_{u \mid s_{0}}+A Q_{u \mid s_{0}} \cdot\left(\sigma_{u \mid s_{0}}^{2}-\sigma_{12 \mid s_{0}}\right.\right. & & \\
& \left.\left.+\mathbf{P}\left(s_{1}\right) \cdot\left(\bar{c}_{u \mid s_{0}}^{2}-\bar{c}_{2 \mid s_{0}} \bar{c}_{1 \mid s_{0}}-\bar{c}_{u \mid s_{0}} \sum_{v=1}^{2} \bar{c}_{v \mid s_{1}} \frac{Q_{v \mid s_{1}}}{Q_{u \mid s_{0}}}\right)\right)\right) \geq 0, & \\
\frac{\partial \mathcal{L}}{\partial K_{2}} & =c_{i n v, 2}-\lambda+\mathbf{P}\left(s_{1}\right) t_{2} \cdot \sum_{u=1}^{2}(-1)^{u} \cdot\left(\bar{c}_{u \mid s_{1}}+A Q_{u \mid s_{1}} \cdot\left(\sigma_{u \mid s_{1}}^{2}-\sigma_{12 \mid s_{1}}\right.\right. & & \\
& \left.\left.+\mathbf{P}\left(s_{0}\right) \cdot\left(\bar{c}_{u \mid s_{1}}^{2}-\bar{c}_{2 \mid s_{1}} \bar{c}_{1 \mid s_{1}}-\bar{c}_{u \mid s_{1}} \sum_{v=1}^{2} \bar{c}_{v \mid s_{0}} \frac{Q_{v \mid s_{0}}}{Q_{u \mid s_{1}}}\right)\right)\right) \geq 0, & & \perp K_{2} \geq 0 \\
\frac{\partial \mathcal{L}}{\partial \lambda} & =D(0)-K_{1}-K_{2} \leq 0, & \perp & \lambda \geq 0
\end{array}
$$

The optimal portfolio mix $\left(K_{1}^{*}, K_{2}^{*}\right)$ to problem (9)-(12) is either obtained as a boundary solution with $K_{1}^{*}=0$ or $K_{2}^{*}=0$ or as an interior solution with $K_{1}^{*}, K_{2}^{*}>0$. The necessary condition for an interior 
solution is determined by the non-convex equation system with Eqs. (44), $\frac{\partial \mathcal{L}}{\partial K_{1}}=0$, and (45), $\frac{\partial \mathcal{L}}{\partial K_{2}}=0$. Both equations represent functions of $K_{1}$ and $K_{2}$, respectively $t_{1}$ and $t_{2}$.

As for the purely cost-minimal portfolio, two cases for $\lambda$ have to be distinguished: For $\lambda=0$, total installed capacity can exceed the maximal demand, i.e. $K_{1}+K_{2} \geq D(0)$, while for $\lambda>0$ total capacity will meet but not exceed the demand maximum, i.e. $K_{1}+K_{2}=D(0)$.

First, we consider the case $\lambda>0$. Here, $\lambda$ can be eliminated in Eqs. (44) and (45) by subtracting $\frac{\partial \mathcal{L}}{\partial K_{1}}-\frac{\partial \mathcal{L}}{\partial K_{2}}=: v$. The resulting optimality condition can then be written as $v=0$ with

$$
\begin{aligned}
v\left(t_{1}\right)= & A \mathbf{P}\left(s_{0}\right) \mathbf{P}\left(s_{1}\right) \sum_{i=0}^{1} t_{i+1}\left(Q_{i+1 \mid s_{i}} \mathbf{E}\left[\tilde{z} \mid s_{i}\right]^{2}-Q_{E}\left(\bar{c}_{2 \mid s_{0}}-\bar{c}_{2 \mid s_{1}}\right) \mathbf{E}\left[\tilde{z} \mid s_{i}\right](-1)^{i}-\mathbf{E}\left[\tilde{z} \mid s_{1}\right] \mathbf{E}\left[\tilde{z} \mid s_{0}\right] Q_{i+1 \mid s_{i}}\right) \\
& +c_{i n v, 1}-c_{i n v, 2}-\mathbf{E}[(t \mid \tilde{s}) \cdot \mathbf{E}[\tilde{z} \mid \tilde{s}]]+A \mathbf{E}\left[(t \mid \tilde{s}) \cdot Q_{1 \mid \tilde{s}} \operatorname{Var}[\tilde{z} \mid \tilde{s}]-Q_{E}\left(\sigma_{2 \mid \tilde{s}}^{2}-\sigma_{12 \mid \tilde{s}}\right)\right]
\end{aligned}
$$

Remark that $v\left(t_{1}\right)$ is solely dependent on the decision variable $t_{1}$, respectively $K_{1}$, if we use the relation $t_{2}\left(t_{1}\right)=R\left(D(0)-D\left(t_{1}\right)\right)$. The combined cost-risk efficient portfolio structure given uncertainty in the merit order with reversal risk $\mathbf{P}\left(s_{1}\right) \geq 0$ can be characterized by the following property as shown in Appendix B.5:

Proposition 2.3 (Existence and uniqueness of an interior solution for the case $\lambda \neq 0$ ). Let be $c_{i n v, 1}>c_{i n v, 2}$ and total installed capacity matching maximum demand, i.e. $\lambda \neq 0$ in constraint (12). If both technologies 1 and 2 are included in the purely cost-efficient portfolio (i.e. $A=0$ ) and in the purely variance-efficient portfolio (i.e. $A \rightarrow+\infty$ ) satisfying condition (42), then all cost-variance efficient portfolios with $A>0$ corresponding to problem (9)-(12) consist of a unique combination of both technologies.

Based on the discussion in Section 2.4, the economic benefit of excess capacities in purely cost-efficient portfolios is limited to investment settings with relatively small investment cost compared to a large difference in operating costs and a severe risk of merit order reversals (see condition (33)). In a risk-cost investment setting, excess capacities are beneficial from an economic point of view if the difference in risk-adjusted expected operating costs is relatively large compared to the investment costs of the generation technologies. Thus, the economic benefit of overcapacities increases with the level of social risk aversion $A$.

Although thus rare in electricity investment applications, we continue to characterize this solution case with $\lambda=0$ in the following propositions (proofs are provided in Appendix B.5):

Proposition 2.4 (Implicit functions). For the implicit function $\frac{\partial \mathcal{L}}{\partial K_{1}}\left(\check{t}_{1}, \check{t}_{2}\right)=0$ with $\frac{\partial \mathcal{L}}{\partial K_{1}}\left(t_{1}, t_{2}\right):[0, T] \times$ $[0, T] \rightarrow Z_{1} \subseteq \mathbb{R}_{+}$, there exists a unique function $\zeta_{1}\left(t_{2}\right):(0, T] \rightarrow Z_{1} \subseteq \mathbb{R}_{+}$. Similarly, for the implicit function $\frac{\partial \mathcal{L}}{\partial K_{2}}\left(\check{t}_{1}, \check{t}_{2}\right)=0$ with $\frac{\partial \mathcal{L}}{\partial K_{2}}\left(t_{1}, t_{2}\right):[0, T] \times[0, T] \rightarrow Z_{2} \subseteq \mathbb{R}_{+}$, there exists a unique function $\zeta_{2}\left(t_{1}\right)$ : $(0, T] \rightarrow Z_{2} \subseteq \mathbb{R}_{+}$ 

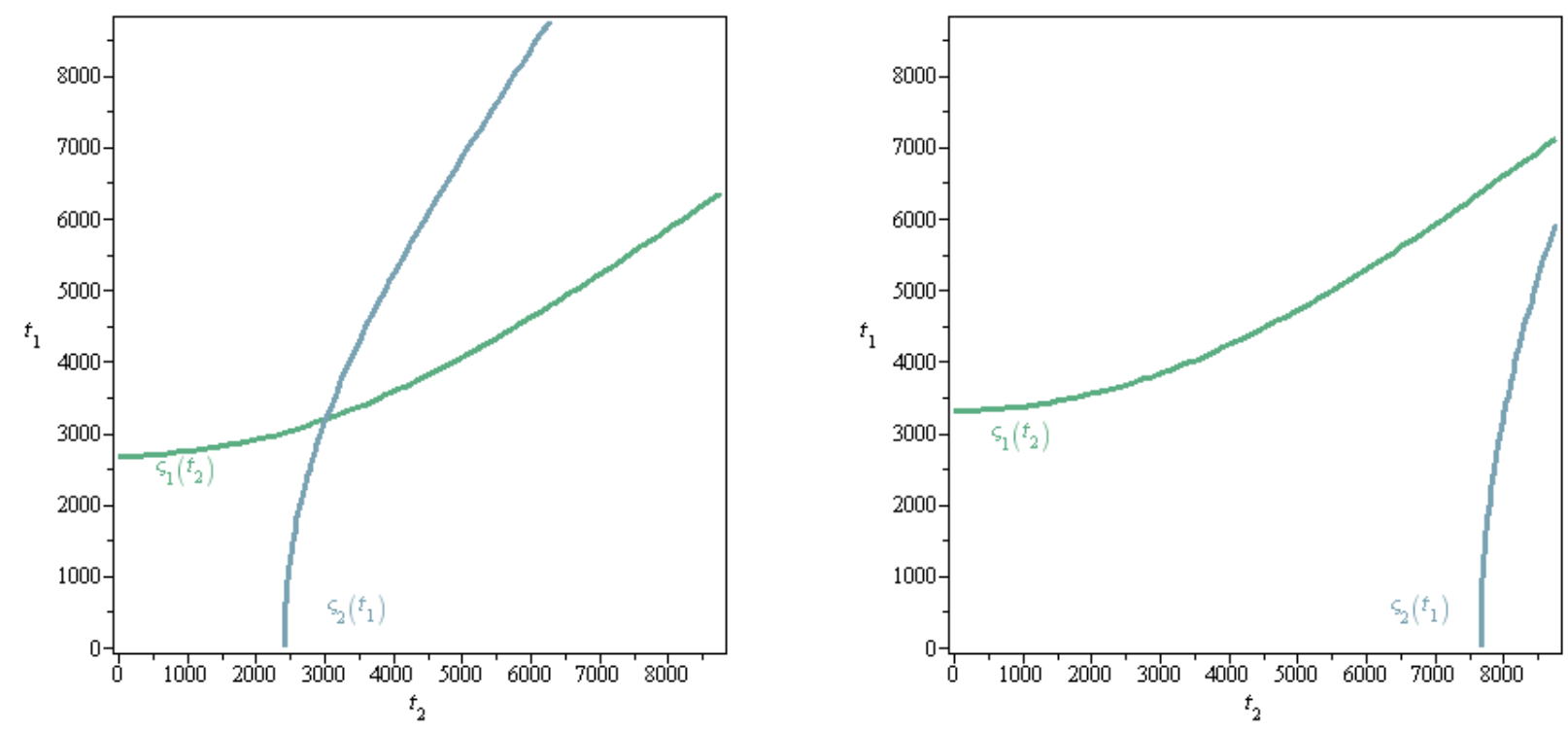

Figure 3: Optimality condition for the portfolio problem as implicit functions of $t_{1}, t_{2}$. In the left diagram, the intersection of $\zeta_{1}\left(t_{2}\right)$ and $\zeta_{2}\left(t_{1}\right)$ represents the solution to the optimization problem for a symmetric, exemplary parameter configuration. For the asymmetric parameter configuration in the right diagram, the problem exhibits a corner solution.

Proposition 2.5 (Monotony). For given expected fuel prices $\bar{c}_{1 \mid s_{0}} \leq \bar{c}_{2 \mid s_{0}}$ and $\bar{c}_{2 \mid s_{1}} \leq \bar{c}_{1 \mid s_{1}}$ and $\zeta_{1}\left(t_{2}\right)$ and $\zeta_{2}\left(t_{1}\right)$ being functions represented by the implicit functions $\frac{\partial \mathcal{L}}{\partial K_{1}}\left(t_{1}, t_{2}\right)=0$ from Eqn. (44), and $\frac{\partial \mathcal{L}}{\partial K_{2}}\left(t_{1}, t_{2}\right)=$ 0 from Eqn. (45), respectively, $\zeta_{1}\left(t_{2}\right)$ and $\zeta_{2}\left(t_{1}\right)$ are both monotone increasing in $t_{2}$ and $t_{1}$, respectively. For $\bar{c}_{1 \mid s_{0}}<\bar{c}_{2 \mid s_{0}}$ and $\bar{c}_{2 \mid s_{1}}<\bar{c}_{1 \mid s_{1}}$, it follows strict monotony of $\zeta_{1}\left(t_{2}\right)$ and $\zeta_{2}\left(t_{1}\right)$.

With $t_{1}=\zeta_{1}\left(t_{2}\right), t_{2}=\zeta_{2}\left(t_{1}\right)$ being the functions represented by the implicit function $\frac{\partial \mathcal{L}}{\partial K_{1}}\left(t_{1}, t_{2}\right)=0$, and $\frac{\partial \mathcal{L}}{\partial K_{2}}\left(t_{1}, t_{2}\right)=0$, respectively, the existence of at least one stationary tuple $\left(t_{1}^{o}, t_{2}^{o}\right) \in(0 ; T) \times(0 ; T)$ with $\zeta_{1}\left(t_{2}^{o}\right)=t_{1}^{o}$ and $\zeta_{2}\left(t_{1}^{o}\right)=t_{2}^{o}$ is necessary prerequisite for an interior solution. This tuple represents graphically the intersection point of $\zeta_{2}\left(t_{2}\right)$ and $\zeta_{1}\left(t_{2}\right)$ as shown in cf. Figure 3 for a typical parameter set. An explicit, analytical solution formulation is however infeasible for the case of a general demand function as both equations contain the objective variables as integration limits in $Q_{u \mid \tilde{s}}$. Thereby, the following solution cases may occur in the case $\lambda=0$ :

I) Corner solution with the efficient portfolio consisting of

i) only one technology, i.e. $K_{1}^{*}=D(0), K_{2}^{*}=0$, or $K_{2}^{*}=D(0), K_{1}^{*}=0$, respectively, ${ }^{8}$

ii) both technologies, i.e. $K_{1}^{*}=K_{2}^{*}=D(0)$,

\footnotetext{
${ }^{8}$ Overcapacities may only be economical if they increase the operating flexibility of the generation portfolio. However, with a boundary solution with only one technology in the portfolio, there is no increase in flexibility and therefore a boundary solution excludes excess capacities
} 
II) Interior solution with the efficient portfolio consisting of both technologies, i.e. $0<K_{1}^{*}, K_{2}^{*} \leq D(0)$.

Proposition 2.6 (Existence and uniqueness of an interior solution for the case $\lambda=0$ ). If the technology parameters satisfy

$$
\begin{aligned}
& \frac{c_{i n v, 1}}{T \mathbf{P}\left(s_{0}\right)}-\mathbf{E}\left[\tilde{z} \mid s_{0}\right]-A Q_{E}\left(\sigma_{2 \mid s_{0}}^{2}-\sigma_{12 \mid s_{0}}+\mathbf{P}\left(s_{1}\right) \mathbf{E}\left[\tilde{z} \mid s_{0}\right]\left(\bar{c}_{2 \mid s_{0}}-\bar{c}_{1 \mid s_{1}}\right)\right)<0 \\
\text { and } \quad & \frac{c_{i n v, 2}}{T \mathbf{P}\left(s_{1}\right)}+\mathbf{E}\left[\tilde{z} \mid s_{1}\right]-A Q_{E}\left(\sigma_{1 \mid s_{1}}^{2}-\sigma_{12 \mid s_{1}}+\mathbf{P}\left(s_{0}\right) \mathbf{E}\left[\tilde{z} \mid s_{1}\right]\left(\bar{c}_{2 \mid s_{0}}-\bar{c}_{1 \mid s_{1}}\right)\right)<0,
\end{aligned}
$$

then the cost-variance efficient portfolios with $A>0$ corresponding to problem (9)-(12) consist of a unique combination of both technologies. The total installed generation capacity of the cost-risk efficient portfolio may exceed total demand, implying $\lambda=0$ in constraint (12).

Notably, if there exists a local minimum of $\mathcal{L}$ in $\left(K_{1}^{*}, K_{2}^{*}, \lambda^{*}\right)$ with $\lambda^{*}=0$ according to Proposition 2.6, the installed capacity in the cost-variance efficient portfolio does not necessarily exceed maximum demand. In addition, there may also exist a local minimum of $\mathcal{L}$ in another point $\left(K_{1}^{* *}, K_{2}^{* *}, \lambda^{* *}\right)$ with $\lambda^{* *}>0$. Finally, the corner points as discussed above have to be checked for optimality due to the non-convexity of the problem.

\section{Likelihood of reversals in the merit order due to fuel price risks}

The probability of a reversal in the merit order depends on the joint distribution of operating $\operatorname{costs} \tilde{c}_{1}, \tilde{c}_{2}$, or more precisely on the distribution of the difference in operating costs $\tilde{z}=\tilde{c}_{2}-\tilde{c}_{1}$. Before we propose a general computation method for the reversal risk over the plant's lifetime, we will briefly discuss the basic calculation technique for the periodical risk in the next section.

\subsection{Front year reversal risk}

Given the distribution of fuel prices in the following period, the risk $\mathbf{P}\left(c_{1}>c_{2}\right)$ for a reversal in the merit order in this period can be computed from the two-dimensional density $\varphi_{1,2}$ as

$$
\mathbf{P}\left(c_{1}>c_{2}\right)=1-\int_{-\infty}^{\infty} \int_{-\infty}^{c_{2}} \varphi_{1,2}\left(c_{1}, c_{2}\right) \mathrm{d} c_{1} \mathrm{~d} c_{2}=1-\int_{-\infty}^{\infty} \int_{c_{1}}^{\infty} \varphi_{1,2}\left(c_{1}, c_{2}\right) \mathrm{d} c_{2} \mathrm{~d} c_{1}
$$

Instead of computing the reversal likelihood directly from Eqn. (50), we can use the more convenient transformation $^{9} \tilde{z}=\tilde{c}_{2}-\tilde{c}_{1}$ with $\mathbf{E}[\tilde{z}]=\bar{c}_{2}-\bar{c}_{1}$ and $\operatorname{Var}[\tilde{z}]=\sigma_{1}^{2}+\sigma_{2}^{2}-2 \sigma_{12}$. In knowledge of the cumulative distribution function of $\Phi(z)$ the likelihood for reversals can be calculated as

$$
\mathbf{P}\left(c_{1}>c_{2}\right)=\mathbf{P}(z<0)=\Phi(0),
$$

\footnotetext{
${ }^{9}$ It is well-known that the sum of $n$ jointly normal distributed random variables $X_{i}$, with $X_{i} \sim \mathcal{N}\left(\mu_{i}, \sigma_{i}^{2}\right)$ is also normal distributed with mean and variance

$$
\mu=\sum_{i=1}^{n} \mu_{i}, \quad \sigma^{2}=\sum_{i=1}^{n} \sum_{j=1}^{n} \sigma_{i j}=\sum_{i=1}^{n} \sum_{j=1}^{n} \sigma_{i} \sigma_{j} \rho, \quad \text { with } \rho=\frac{\sigma_{i j}}{\sigma_{i} \sigma_{j}} .
$$
}

For a proof see e.g. Elishakoff (1999). 


\subsection{Reversal risk over the plant's lifetime}

Up to now, we have calculated the probability $\mathbf{P}\left(c_{2}<c_{1}\right)$ for a single realization of fuel prices such that operating costs of technology 2 exceed those of technology 1. In fact, this calculation captures only the risk of a reversal in a period $\tau+1$ given all information at $\tau$, or more precisely $\mathbf{P}_{\tau}\left(c_{2, \tau+1}<c_{1, \tau+1}\right):=$ $\mathbf{P}\left(c_{2, \tau+1}<c_{1, \tau+1} \mid \mathcal{F}_{\tau}\right)$. Beyond that, the investment decision requires to take into account the reversal risk in all subsequent periods of the plants' lifetime. Valuing the optimal fuel mix over all periods of the considered plant portfolio would in general require a relatively complex multi-period model, but since the investment is limited to one point in time, the problem can be reduced to a single-period model by calculating the average reversal risk over all periods of the plants' lifetime.

It is intuitively clear that the likelihood for a reversal in the merit order multiple periods ahead depends on the type of the assumed underlying stochastic fuel price process and as a consequence on the type of the resulting process of differences in operating costs. To study the impact on the reversal risk over the plant's lifetime, we will discuss in the following two fundamental stochastic processes which are typically applied in financial energy-related applications:

First, let differences in periodic operating costs $\tilde{z}_{\tau}=\tilde{c}_{\tau, 2}-\tilde{c}_{\tau, 1}$ be represented by a random walk defined on the probability space $\left(\Omega, \mathcal{F}_{\tau}, \mathbf{P}\right)$ of the form

$$
\Delta z_{\tau}=\sigma \varepsilon_{\tau} \sqrt{\Delta \tau}
$$

where $\varepsilon$ denotes the standardized white noise with $\varepsilon_{\tau} \stackrel{i . i . d .}{\sim} \mathcal{N}(0,1), \tau \in \mathbb{N}$. Since the distribution of $z_{\tau+k}$ given all information at time $t$ is non-stationary with constant mean but linearly increasing variance $\operatorname{Var}_{t}\left[z_{\tau+k}\right]=k \cdot \sigma^{2}$, the likelihood for a reversal in the merit order $k$ periods ahead will also increase with $k$ as shown in Figure 4. Typical solution approaches for these kinds of problems apply multi-period optimization frameworks. To keep the simplicity of the annualized valuation framework proposed in Section 2, we use instead the compound periodical likelihood of reversals, calculated as the weighted average of the single-period reversal probabilities during the lifetime $\tau \in[1, \ldots, \hat{\tau}]$ of the considered plants. Thereby, the discount factor is used as weighting factor, i.e.

$$
\mathbf{P}\left(\tilde{s}=s_{1}\right) \equiv \mathbf{P}\left(z_{[1, \hat{\tau}]}<0\right)=\frac{q^{\hat{\tau}} \cdot i}{q^{\hat{\tau}}-1} \cdot \sum_{k=1}^{\hat{\tau}} \mathbf{P}\left(z_{k}<0\right) \cdot q^{-k}
$$

with $q=1+i$ and $i$ denoting the discount rate.

While the random walk model excludes any predictability of the difference in operating costs of the two technologies, application of a mean-reversion model follows the idea that there is a long-term equilibrium for both technologies. This rationale can be motivated with the long-term substituting effects of commodities in many industries and is supported by various studies on cointegration of commodity prices (cf. e.g. Schwartz, 1997; Alexander, 1999; Pindyck, 1999; Schwartz and Smith, 2000; Pindyck, 2001; Geman, 2007; 

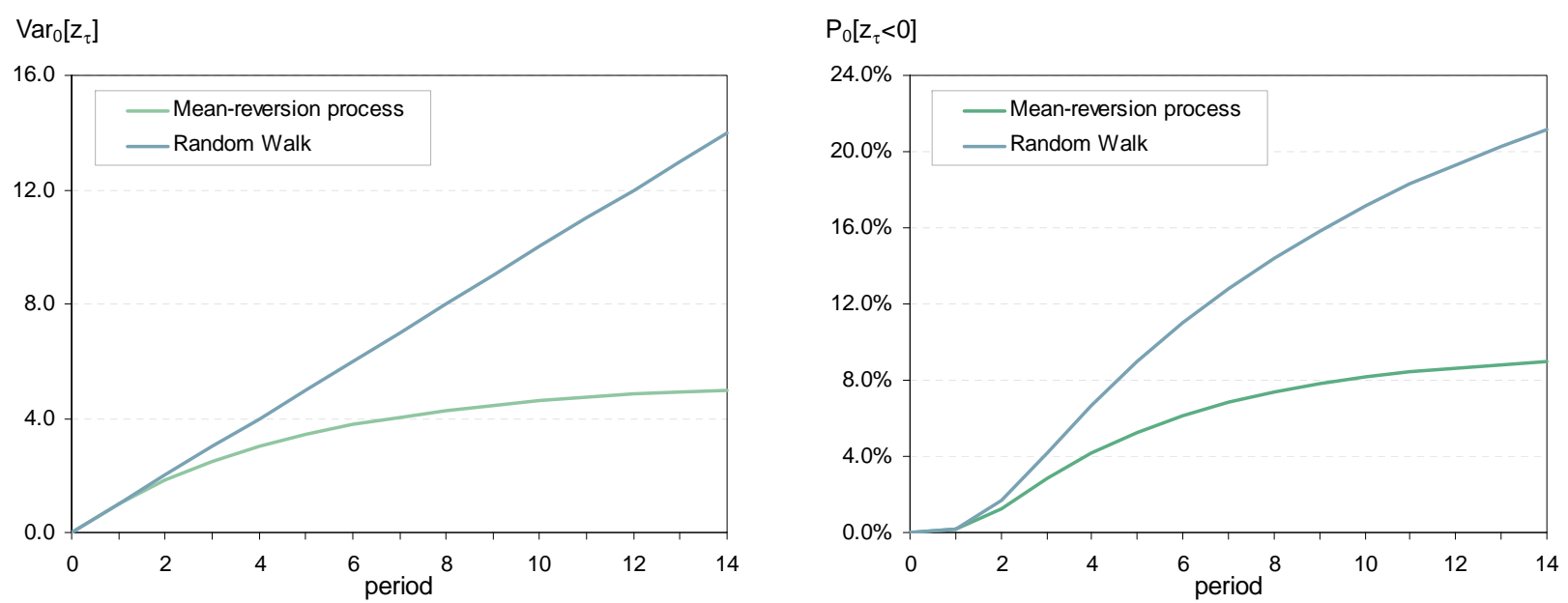

Figure 4: Comparison of variance $\operatorname{Var}_{0}\left[z_{t}\right]$ (left) and corresponding periodical reversal risk for $\mathbf{P}_{0}\left(\tilde{z}_{t}<0\right)$ (right) for a random walk and a mean-reversion process $(\theta=0.8)$. The expected mean difference is $\mathbf{E}[z]=3$ and the standard deviation $\sigma=1$ for both processes.

Mohammadi, 2009). In fact, mean-reverting behavior of the differences of two stochastic processes does even imply cointegration, i.e. stationarity of a linear combination of two stochastic processes. In addition to short-term deviations of operating costs caused by fluctuations in supply and demand of the underlying fuel types, variations in the long-run equilibrium may occur caused e.g. by technological progress impacting investment costs of generation technologies. Hence, the distribution of price differences in operating costs of two technologies is usually time-dependent and not constant over time.

We consider a mean-reversion process (corresponding to an $\mathrm{AR}(1)$ time series model) on the probability space $\left(\Omega, \mathcal{F}_{t}, \mathbf{P}\right)$ of the form

$$
\Delta z_{\tau}=\theta\left(\mu-z_{\tau}\right) \Delta \tau+\sigma \varepsilon_{\tau} \sqrt{\Delta \tau}
$$

where $\varepsilon_{\tau}$ is again assumed to be the standardized white noise. For $|\theta|<1$ and $\Delta \tau \rightarrow \infty$, the process is (weakly) stationary with bounded variance $\operatorname{Var}\left[z_{\tau}\right]=\frac{\sigma_{\epsilon}^{2}}{2 \theta}$ and constant expectation $\mathbf{E}\left[z_{\tau}\right]=\mu$. As a consequence, the periodical likelihood for a reversal in the merit order given mean-reverting difference in operating costs is also limited to the reversal risk implied by the variance supremum (see Figure 4). If the mean reversion parameter $\theta$ is sufficiently small, this upper bound may also be used as a fair approximation for the periodical reversal likelihood, i.e.

$$
\mathbf{P}\left(\tilde{s}=s_{1}\right) \equiv \mathbf{P}\left(z_{[1, \hat{\tau}]}<0\right) \approx \Phi_{z_{\hat{\tau}}}(0)
$$

where $\Phi_{z_{\hat{\tau}}}$ is the unconditional cumulated normal probability distribution of $z_{\hat{\tau}}$ with $\tilde{z}_{\hat{\tau}} \sim \mathcal{N}\left(\mu, \frac{\sigma_{\epsilon}^{2}}{2 \theta}\right)$. 


\section{Application: Optimal generation portfolios of coal and CCGT technologies for the German electricity market}

To illustrate the results, the proposed model is calibrated on the German electricity market using historical market data. For that, typical new CCGT and hard coal technologies are considered for serving demand. Note that the purpose of the calibration is to allow us to derive practically relevant results. The numbers thereby serve primarily as an illustration whereas this paper does not claim to derive a complete picture on the efficient power generation fuel mix.

\subsection{Estimation of model parameters}

Economic and technical key parameters of the coal and CCGT plant technologies based on Konstantin (2009) are depicted in Table 1. Total operating costs are calculated based on fuel, $\mathrm{CO}_{2}$ emission, and variable O\&M costs. To account for fuel price risks, total operating costs $\tilde{c}_{u}$ are modeled as normally distributed random variables calculated as the sum of the respective fuel prices plus the emission factor weighted price of $\mathrm{CO}_{2}$ emission rights divided by the technology specific efficiency rate, i.e. $c_{u}=\left(p_{f, u}+e_{u} p_{c o_{2}}\right) / \eta_{u}$. A two-step approach is used to determine the likelihood for reversals in the merit order and the conditional distribution parameters as described in further detail in the following paragraphs:

- In the first step, the compound periodical likelihood for reversals in the merit order is determined based on the historical difference time series in operating costs of coal and CCGT technologies.

- In the second step, conditional distribution parameters for the individual time series of operating costs of coal and CCGT are computed based on the corresponding unconditional distribution parameters and the likelihood for reversals in the merit order.

Table 1: 2007 based key parameters for new conventional coal and CCGT technologies (source: Konstantin, 2009, own analysis).

\begin{tabular}{llll}
\hline Parameter & Unit & Hard coal & CCGT \\
\hline Total net investment costs & $€ / \mathrm{KW}$ & 1419 & 608 \\
Technical lifetime & $\mathrm{a}$ & 45 & 30 \\
Fixed O\&M, overhead & $€ / \mathrm{KW} \mathrm{a}$ & 36.06 & 13.97 \\
Annualized investment costs $c_{i n v}$ & $€ / \mathrm{KW}$ & 179.905 & 78.442 \\
\hline Variable O\&M, transport & $€ / \mathrm{KWh}_{e}$ & 2.9 & 5.5 \\
Thermal efficiency & $\mathrm{MWh}_{e} / \mathrm{MWh}_{t}$ & 0.46 & 0.56 \\
Carbon emission rate & $\mathrm{tCO}_{2} / \mathrm{MWh}_{t}$ & 0.34 & 0.20 \\
\hline
\end{tabular}




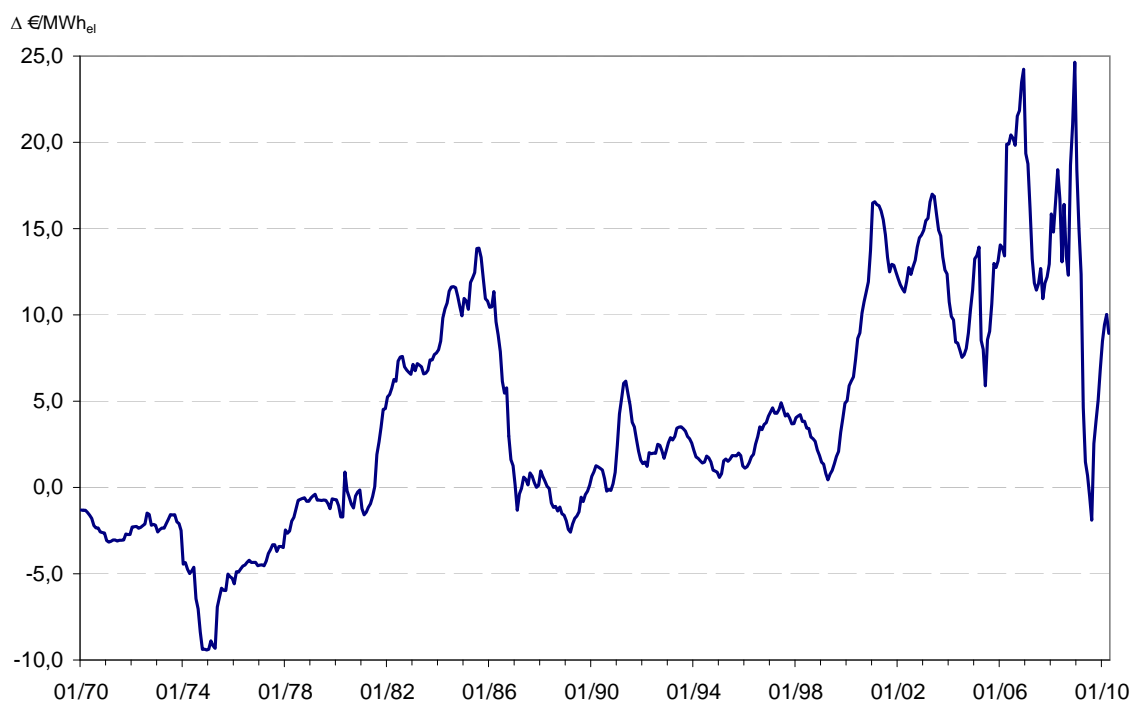

Figure 5: Differences in total operating costs of new CCGT and hard coal plants in Germany 1970-2010 (source: BAFA (2010); StaBu (2010); EEX (2011); own analysis).

\subsubsection{Estimation of the risk of reversals in the merit order}

Time series of monthly coal and natural gas import prices 1970-2010 are used based on the price indices provided by the German Federal Statistical Office (StaBu, 2010) and absolute data of the German Federal Office of Economics and Export Control (BAFA, 2010). The price data reflects the average cross-border price converted to $€ / \mathrm{MWh}_{t}$ for all contracted deliveries in the respective month. Starting with the beginning of the European Union Emission Trading System in 2005, total fuel prices are computed including the costs of $\mathrm{CO}_{2}$ emission allowances (EUA) based on front year price data from EEX (2011). EUAs are modeled to be purchased at market conditions (full auctioning) as it has been announced by the EU for ETS Phase III starting in 2013. Levels of differences in variable generation costs of new CCGT and hard coal technologies are computed from the nominal time series, i.e. $z_{\tau}=c_{\tau, c c g t}-c_{\tau, \text { coal }}$, as shown in Figure $5 .{ }^{10}$

The difference time series is then analyzed with respect to random walk and mean reversion properties: Table 2 provides "regular" and augmented Dickey-Fuller (ADF) test statistics on unit roots of the difference time series $z_{\tau}$. While the DF test statistic does not allow to reject the null hypothesis of unit roots, the $\mathrm{ADF}$ test allows a rejection at a weak $10 \%$ level indicating trend-stationary time series characteristics. Following from both tests results, non-stationarity for the differences time series in operating costs cannot be excluded. However, this hypothesis is conflicting with the principles of a long-term market equilibrium: Since the gap in operating costs will in the long-run influence new built decisions of power plant investors

\footnotetext{
${ }^{10}$ Instead of using nominal data, we also considered deflating the data into real terms. This methodology, however, may yield biasing results since selection of an appropriate deflator is an ambiguous process. Having tested wholesale price indices for deflation, the time series properties of the difference s in operating costs did not much change.
} 
Table 2: Regular and augmented Dickey-Fuller tests on unit roots and estimated parameters for the difference time series of variable generation costs $z_{\tau}=c_{\tau, \text { ccgt }}-c_{\tau, \text { coal }}, 1970-2010$. The reversal likelihoods are based on the long-term mean difference in operating costs 1970-2010 for $\mathbf{P}_{l}\left(\tilde{s}=s_{1}\right)$ and on the short-term period 2007-2009 for $\mathbf{P}_{s}\left(\tilde{s}=s_{1}\right)$, respectively.

\begin{tabular}{|c|c|c|c|c|c|c|c|}
\hline \multirow{2}{*}{ Time series $z_{\tau}$} & \multicolumn{2}{|c|}{ Test statistic } & \multicolumn{3}{|c|}{ Parameter estimates ( $t$-statistics) } & \multicolumn{2}{|c|}{ Reversal risk } \\
\hline & $\mathrm{DF}$ & $\mathrm{ADF}$ & $\theta$ & $\mu$ & $\sigma_{\varepsilon}$ & $\mathbf{P}_{l}\left(s_{1}\right)$ & $\mathbf{P}_{s}\left(s_{1}\right)$ \\
\hline Mean reversion & -1.772 & $-3.171^{*}$ & $0.013^{*}(1.772)$ & $5.667(1.268)$ & 1.088 & 0.202 & 0.038 \\
\hline Random walk & & & - & $3.988(0.583)$ & 6.840 & 0.401 & 0.240 \\
\hline
\end{tabular}

as well as substituting effects in other industries, a mean-reverting behavior in the differences in operating costs would to be expected in the long-term market equilibrium.

To compare the impact of a mean-reverting process versus a random walk assumption for the difference time series in operating costs with regard to the efficient capacity allocation, the subsequent analysis is carried out applying both types of time series. ${ }^{11}$ Parameter estimates and $t$-statistics are also depicted in Table 2 for annual levels of gas and coal import prices 1970-2009. Correspondingly, the table provides the computed lifetime annuities for a merit order reversal of the coal and CCGT technologies calculated according to Eqs. (53) and (55). Thereby, $\mathbf{P}_{l}\left(\tilde{s}=s_{1}\right)$ denotes the likelihood for reversals under the long-term mean difference in operating costs, $\bar{z}$, over the full estimation period 1970-2009. In contrast, $\mathbf{P}_{s}\left(\tilde{s}=s_{1}\right)$ is based on the mean difference in operating costs $\bar{z}$ estimated from the short-term period 2007-2009. Using the short-term period for estimating mean operating costs is most suitable in our view since it ensures appropriate long-term estimates for variance and covariance while it takes into account recent shifts in the means of operating costs. The long-term estimate for expected operating costs would yield in contrast severe inconsistencies with respect to the other key technology parameters which refer to new built power plants based on recent data. Thus, we use $\mathbf{P}_{s}\left(\tilde{s}=s_{1}\right)$ to accomplish the further analysis. As expected, the limiting periodical reversal likelihood given mean-reverting differences in operating costs is with $3.8 \%$ much lower compared to compound periodic reversal risk of $24.0 \%$ under the random walk hypothesis.

\subsubsection{Unconditional and conditional distribution of operating costs}

Having determined the compound reversal likelihood during the plant's lifetime from the difference time series of operating costs, the corresponding unconditional mean and variance are determined for each time series of operating cost both under the mean reversion and random walk hypothesis. Next, conditional means and variances are computed for each technology as shown in Appendix B.1 (cf. Table 3).

\footnotetext{
${ }^{11}$ Further research remains necessary to provide empirical evidence on the question of random walk versus mean-reverting time series behavior of difference of operating costs. Since the main objective of this section is to provide an illustrative application of the analytical discussion, we kindly refer the reader to existing literature on this topic as mentioned above.
} 
Table 3: Distribution parameters for operating costs of coal and CCGT technologies. Unconditionally expected operating costs represent historical mean costs 2007-2009. Conditional distribution parameters were calculated based on the reversal likelihoods given a random walk and a mean reversion process.

\begin{tabular}{lrrr}
\hline & Empirical estimate & Random walk & Mean reversion \\
\hline $\mathbf{E}\left[\tilde{c}_{\text {coal }}\right]$ & 45.912 & 45.912 & 45.912 \\
$\mathbf{E}\left[\tilde{c}_{c c g t}\right]$ & 57.975 & 57.975 & 57.957 \\
$\mathbf{P}_{s}\left(\tilde{s}=s_{1}\right)$ & - & 0.238 & 0.0383 \\
$\operatorname{Var}\left[\tilde{c}_{c o a l}\right]$ & 84.447 & 513.422 & 83.305 \\
$\operatorname{Var}\left[\tilde{c}_{c c g t}\right]$ & 195.574 & 1189.223 & 192.957 \\
$\operatorname{Cov}\left[\tilde{c}_{\text {coal }}, \tilde{c}_{c c g t}\right]$ & 116.561 & 188.858 & 115.779 \\
$\mathbf{E}\left[\tilde{c}_{\text {coal }} \mid s_{0}\right]$ & - & 50.606 & 46.315 \\
$\mathbf{E}\left[\tilde{c}_{\text {coal }} \mid s_{1}\right]$ & - & 30.871 & 35.795 \\
$\mathbf{E}\left[\tilde{c}_{c c g t} \mid s_{0}\right]$ & - & 69.505 & 58.958 \\
$\mathbf{E}\left[\tilde{c}_{c c g t} \mid s_{1}\right]$ & - & 20.952 & 33.067 \\
$\operatorname{Var}\left[\tilde{c}_{c o a l} \mid s_{0}\right]$ & - & 452.673 & 79.818 \\
$\operatorname{Var}\left[\tilde{c}_{c o a l} \mid s_{1}\right]$ & - & 411.257 & 64.404 \\
$\operatorname{Var}\left[\tilde{c}_{c c g t} \mid s_{0}\right]$ & - & 821.499 & 171.853 \\
$\operatorname{Var}\left[\tilde{c}_{c c g t} \mid s_{1}\right]$ & - & 570.799 & 78.548 \\
$\operatorname{Cov}\left[\tilde{c}_{c o a l}, \tilde{c}_{c c g t} \mid s_{0}\right]$ & - & 559.261 & 106.415 \\
$\operatorname{Cov}\left[\tilde{c}_{c o a l}, \tilde{c}_{c c g t} \mid s_{1}\right]$ & - & 457.363 & 68.49 \\
\hline
\end{tabular}

\subsubsection{Specification of the load duration curve}

The estimation of a load duration function is performed as described in Sunderkötter and Weber (2009): Historical load data for Germany provided in an hourly resolution by ENTSO-E (2009) for the years 20062008 provide the basis for the fitting procedure. For comparability reasons, we adjust the data sets for the general increase in energy consumption by $1.02 \%$ in 2007 and $0.4 \%$ in 2008, respectively. A historical reference load duration curve is then generated from the hourly means of the historic data. To accomplish the further analysis in Matlab with a continuous inverted load duration function $\hat{R}(K)=\hat{D}^{-1}(t)$, we use OLS regression to fit a polynomial function of the form

$$
\tilde{R}(K) \approx \begin{cases}\sum_{j=0}^{q} A_{j} \cdot K^{j}, & \text { for } K \geq D(T) \\ T, & \text { for } K<D(T) .\end{cases}
$$

with $T=8760$ hours and for a load ranging from $D(T)=35031 \mathrm{MW}$ to $D(0)=78332 \mathrm{MW}$. Parameter estimates for a polynomial function of degree $q=7$ are provided in Fig. 6 .

\subsection{Results I: The cost and variance efficient portfolio fuel mix}

We start interpreting the results by first investigating the efficient technology mix under the two extreme cases with $A=0$ and $A \rightarrow \infty$. 


\section{Load duration curve}

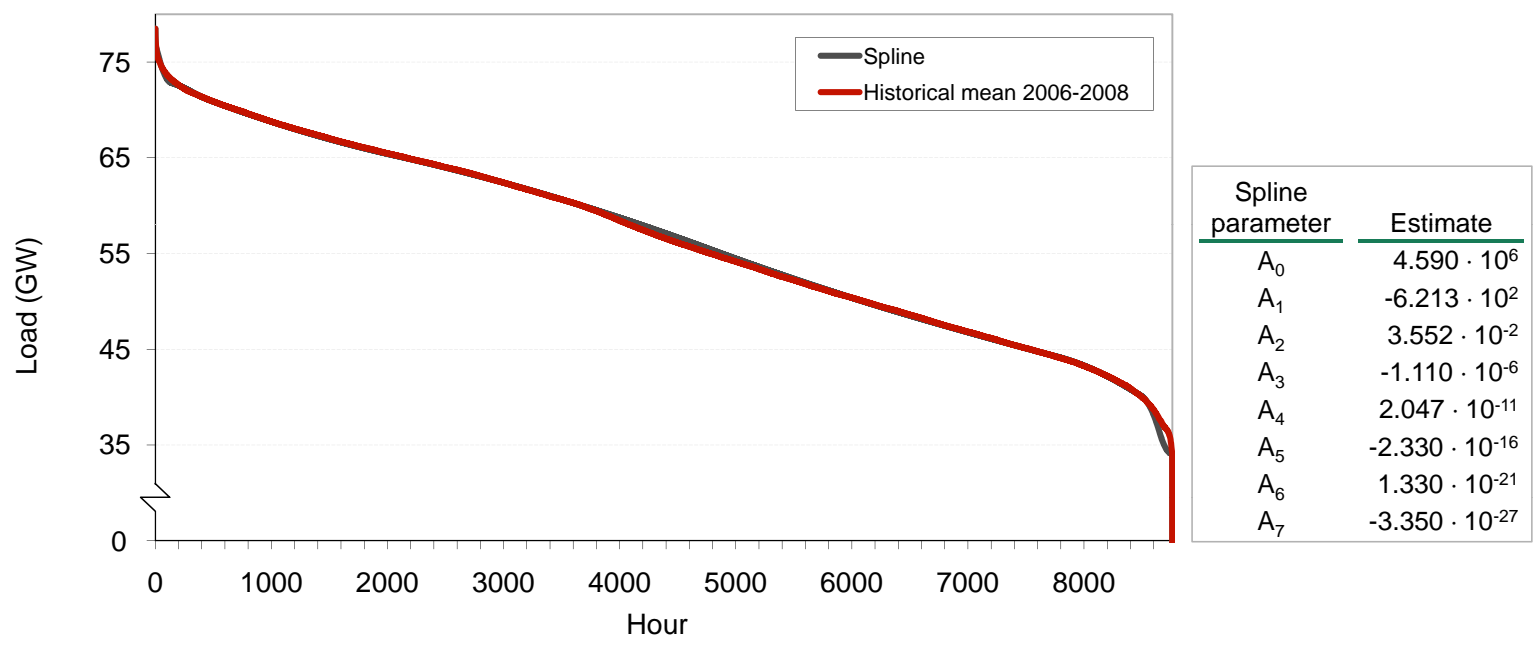

Figure 6: Historical and fitted load duration curve and parameter specification of the polynomially fitted inverse load duration function $R(K)=D^{-1}(t)$ (ENTSO-E, 2009, own analysis).

In a first approximation, the risk for reversals in the merit order may be neglected to assess the solution case for the efficient portfolio structure as discussed in Section 2.3. Assuming risk-neutrality (i.e. $A=0$ ) given the estimated technology parameterization (cf. Tab. 3), the cost efficient portfolio includes both generation technologies according to Property 2.1 , since it holds $\left(c_{\text {inv }, 1}-c_{\text {inv }, 2}\right)\left(\bar{c}_{2}-\bar{c}_{1}\right)=8411 \in(0 ; 8760)$. The purely cost efficient portfolio (i.e. $A=0$ ) consists of a balanced mix of both technologies with about $48 \%$ CCGT and 52\% hard coal capacity (Fig. 7). It can be seen that the cost efficient technology mix both under the random walk and the mean-reversion assumption is fairly well approximated by the calculation with neglected risk of reversals in the merit order. The absolute discrepancy between the calculations with and without reversal risk is marginal.

From a pure risk perspective, a comparison of the unconditional variance of operating costs shows the superiority of coal compared to the CCGT technology. Neglecting the risk for reversals in the merit order and applying Property 2.2, it can bee seen that the variance efficient portfolio will only contain coal technologies since $\sigma_{1}^{2}-\sigma_{12}=84.447-116.561<0$. The picture does not change by taking into account the risk for reversals in the merit order: Under the random walk assumption, the conditional variance of the CCGT technology exceeds the conditional variance of the coal technology in both merit order states. Put differently, the coal technology dominates the CCGT technology with respect to the scenario variance. Hence, diversification is not efficient. As expected, the sufficient condition (42) for variance efficient portfolios including both technologies as formulated in Property 2.2 is not satisfied. 

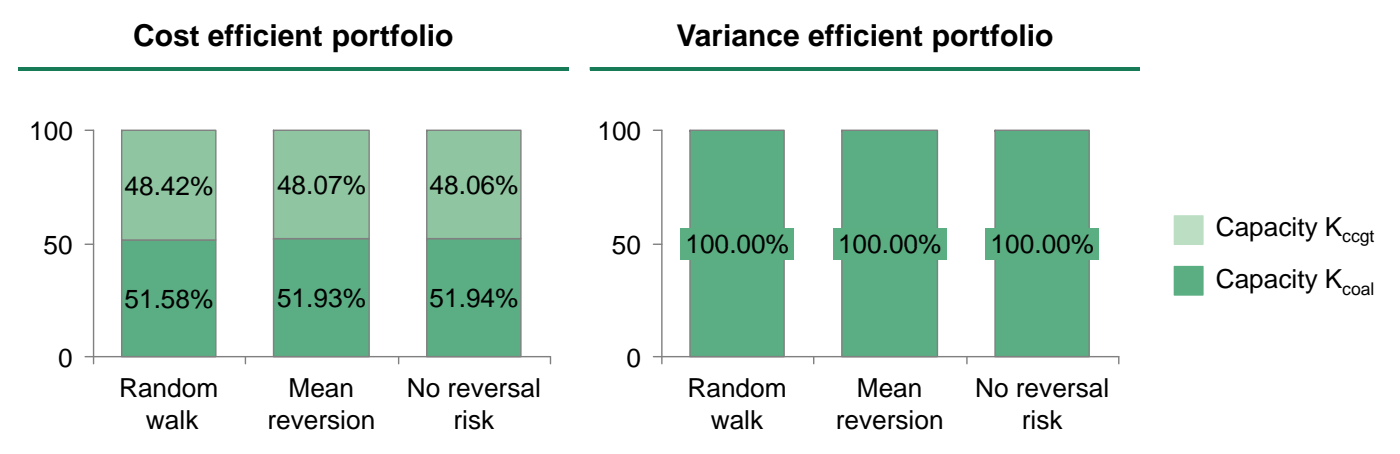

Figure 7: Capacity structure of purely cost and variance efficient generation portfolios consisting of new CCGT and hard coal technologies under the random walk and mean reversion hypothesis and for the approximating calculation with neglected reversal risk.

\subsection{Results II: Cost-risk efficient fuel mix under the random walk and the mean reversion hypothesis}

To assess the technology structure of cost-risk efficient portfolios, we compute efficient capacities and total system costs of hard coal and CCGT technologies for levels of risk aversion in a range of $A=\left[0, \ldots, 10^{-9}\right]$ under the two alternative assumption that differences in operating costs follow a random walk or a mean reversion process.

Figure 8 presents the efficient capacity mix of CCGT and hard coal technologies under the assumption that differences in operating costs follow a random walk. Correspondingly, Figure 9 depicts the efficient portfolio structure under the mean-reversion assumption. For reference purposes, the hypothetical efficient portfolio structure without reversal risk is indicated by a dashed line.

Despite the relatively high risk for reversals in the merit order under the random walk assumption, the share of CCGT generation in the efficient portfolio is decreasing with increasing levels of risk aversion. This is due to the specific risks of coal and CCGT technologies: The higher the risk aversion factor $A$, the stronger is the impact of the variance in the objective function. With the risk contribution of CCGT technologies being higher than that of coal technologies, the higher overall risk in the case with reversal risk implies that the efficient portfolios contain higher shares of coal generation compared to the calculations without reversal risk.

Under the random walk hypothesis, the share of coal generation in efficient generation portfolios is much higher than under a mean-reverting difference time series of operating costs. This is due to the fact that the higher probability of reversals in the merit order results also in a higher absolute portfolio risk (measured both by conditional and unconditional variances) than under the mean-reversion assumption. Due to the relatively small reversal risk in the latter case, the efficient portfolio structure under the mean-reversion assumption is fairly well approximated by a model formulation with neglected reversal risks.

Remarkably, neither the efficient portfolios under the random walk nor under the mean reversion hypothesis exhibit any overcapacities in the numerical example. This phenomenon will be analyzed in detail 


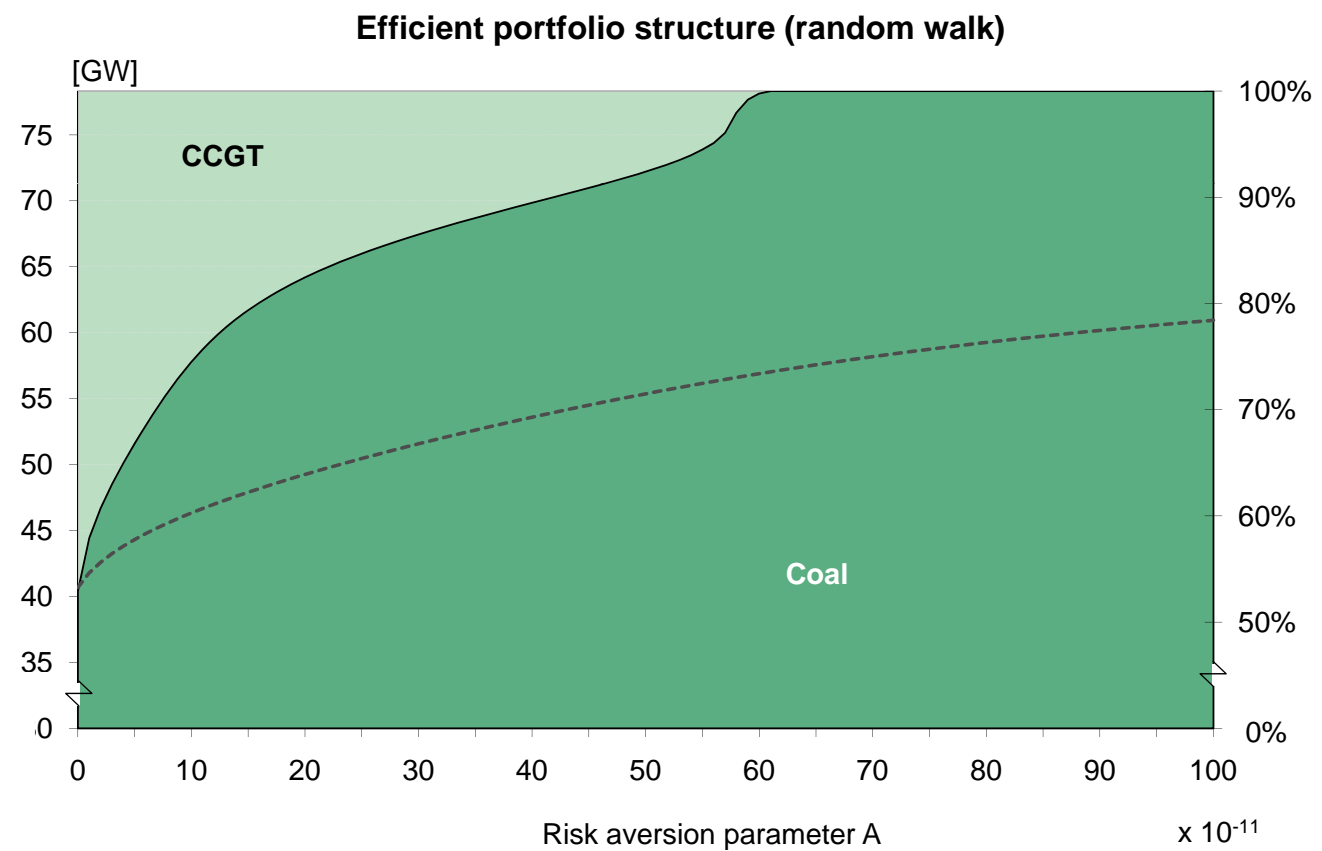

Figure 8: Capacity structure of efficient generation portfolios consisting of new CCGT and hard coal technologies for varying risk aversion parameter, $A$, given that differences in operating costs follow a random walk. The hypothetical efficient portfolio structure without reversal risk is shown by the dashed line.

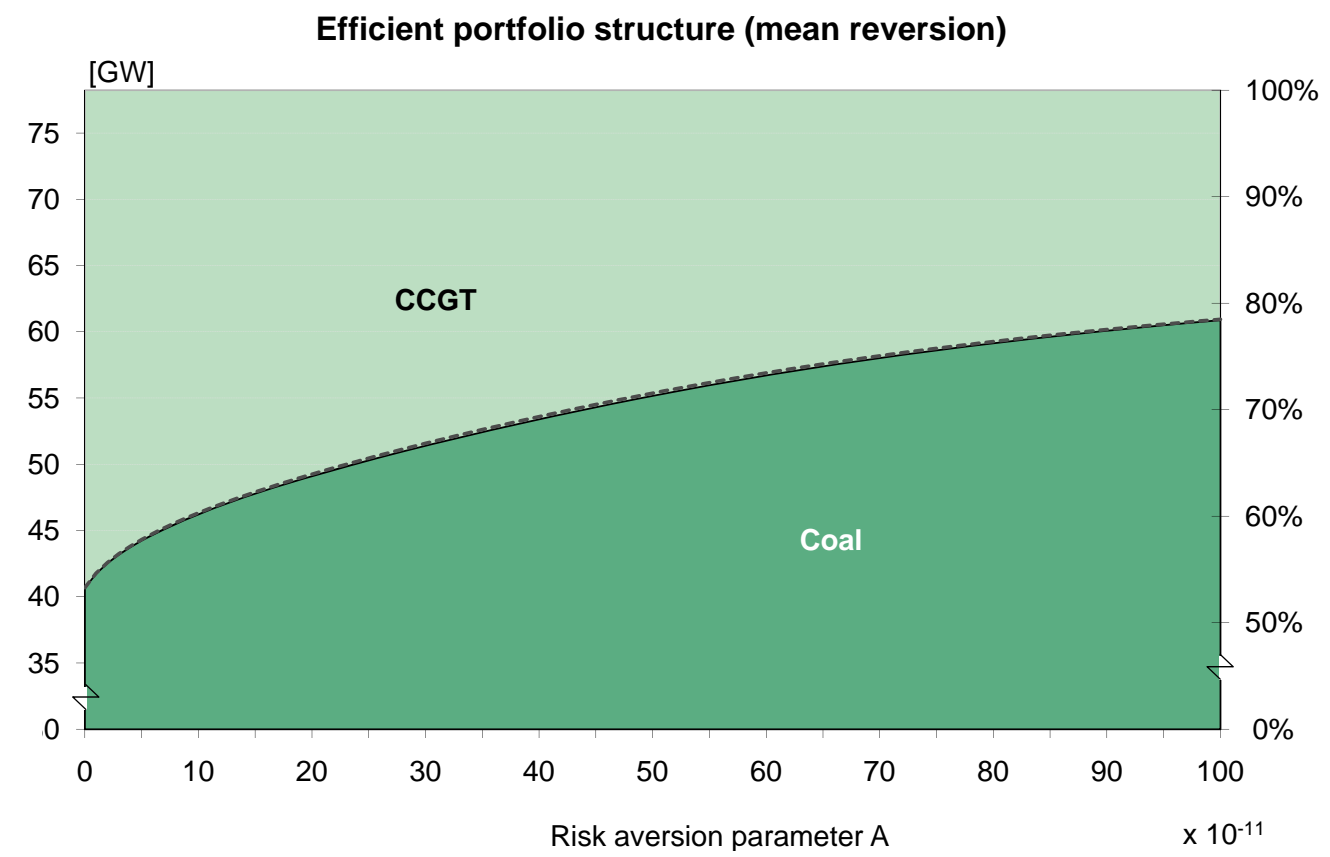

Figure 9: Capacity structure of efficient generation portfolios consisting of new CCGT and hard coal technologies for varying risk aversion parameter, $A$, given that differences in operating costs follow a mean-reversion process. The hypothetical efficient portfolio structure without reversal risk is shown by the dashed line. 
in the following paragraph.

\subsection{Results III: Overcapacities in efficient portfolios}

In a world with deterministic peak demand and full information about plant availabilities, there is no need to install more generation capacity than maximum demand as long as uncertainty of generation costs does not lead to changes in the merit order.

The picture may change drastically given uncertainty in the merit order as already discussed earlier in this paper: If there is a substantial risk that the CCGT plant may run as the base-load plant in certain periods, then it may be economical to install a higher share of CCGT generation capacity compared to the situation where CCGT is only expected to run as peak plant. Thereby, the question whether to build more generation capacity than maximum demand depends highly on the relation of capacity investment costs compared to the difference of operating costs: The lower plant-specific investment costs, the more economical it becomes to build overcapacities. Considering the extreme case with zero capacity investment costs, the cost-minimal generation portfolio would include generation capacities of each technology at maximum demand, i.e. the total installed capacity would be twice the maximum demand.

For the case with risk-neutrality (i.e. $A=0$ ), a necessary parameter condition for plant overcapacities has been formulated in Eqn. (33). For this case, the interdependency between total excess capacity (measured by the system capacity ratio which equals total installed capacity divided by maximal demand) and specific investment costs is shown in Figure 10 (left): While the efficient portfolio does not include any overcapacity for the empirically estimated plant investment costs (indicated by the red stack), the system capacity ratio increases up to $200 \%$ for decreasing investment costs. Figure 10 (right) indicates at which parameter combination the necessary condition for overcapacities, Eqn. (33), is satisfied.

For the risk-extended portfolio problem, one could expect that the system capacity ratio would increase the higher the levels of risk aversion. However, the opposite can be observed as shown in Figure 11 assuming that operating costs follow a random walk: With increasing societal risk aversion $A$, the installed overcapacity in efficient portfolios decreases. This is at first sight contra-intuitive as one would expect that with increasing risk aversion, the limiting impact of investment costs on the portfolio diversification decreases in favor of a more flexible plant portfolio. However, the reason is again the specific risk of the coal and the CCGT technologies: The risk contribution of the CCGT technology is higher compared to the coal technology. Thus, the higher the risk aversion factor $A$, the greater is the impact of the variance in the objective function (5) and hence the less attractive is diversification into the CCGT technology.

\subsection{Results IV: Impact of increased risk of the coal technology}

Based on the historically estimated operating costs parameters (cf. Tab. 3), the coal technology is superior compared to the CCGT technology from a pure risk perspective (measured by variance of operating 

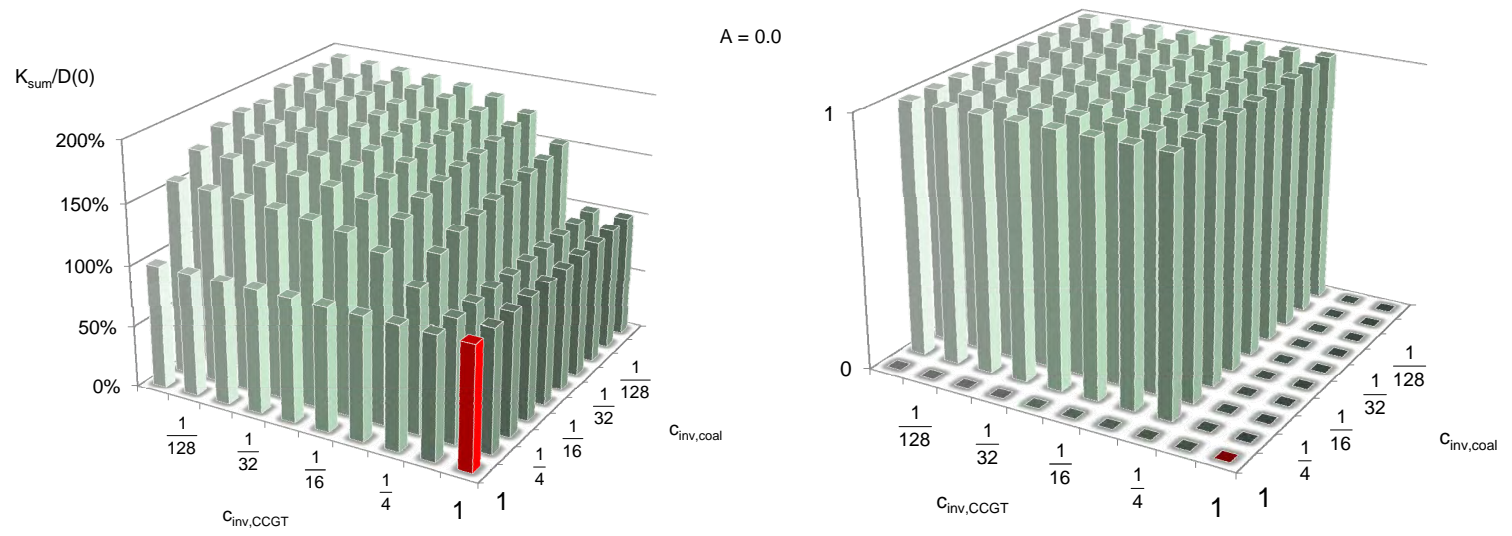

Figure 10: System capacity ratio $\left(K_{\text {coal }}^{*}+K_{C C G T}^{*}\right) / D(0)$ for varying specific investment costs of coal and CCGT technologies for the purely cost efficient portfolio, i.e. $A=0$ (left). The red stack represents the efficient capacity for the empirically derived annual investment costs. The graph on the right indicates, for which parameter combinations the necessary condition for overcapacities discussed in Eqn. (33) is satisfied.
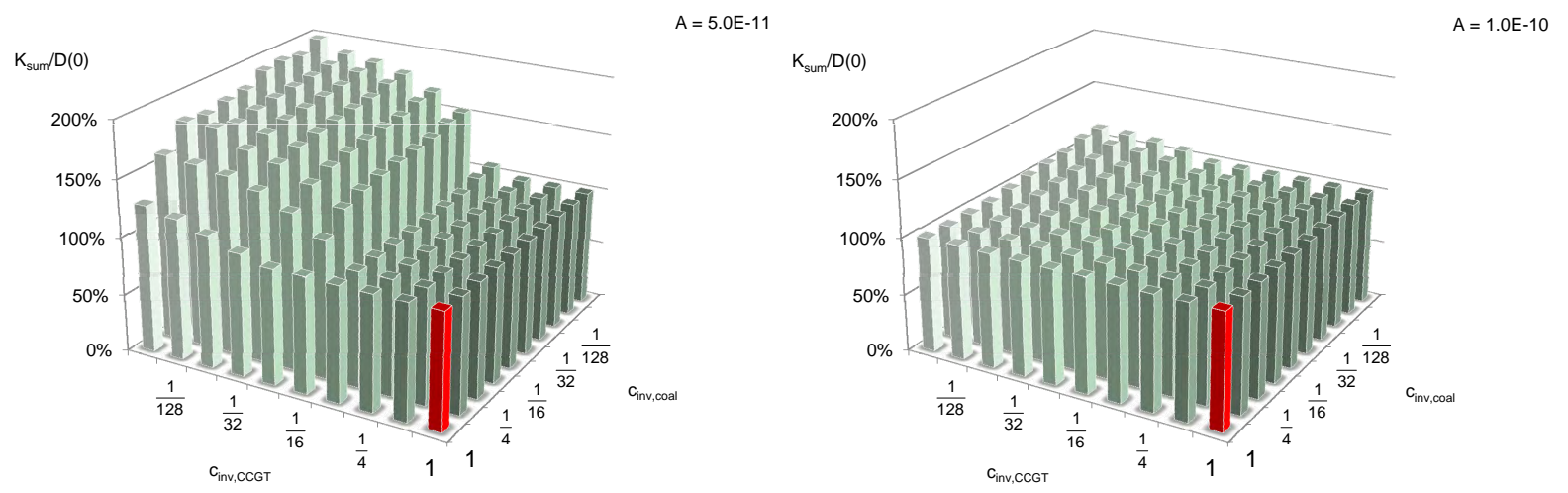

Figure 11: System capacity ratio $\left(K_{\text {coal }}^{*}+K_{C C G T}^{*}\right) / D(0)$ for varying specific investment costs of coal and CCGT technologies at risk aversion levels $A=5.0 \cdot 10^{-11}$ (left), and $A=1.0 \cdot 10^{-10}$ (right). Differences of operating costs are assumed to follow a random walk. The red stack represents the efficient capacity for the empirically derived annual investment costs.

costs). Yet, the variance of operating costs of the considered technologies is driven by changes of the fuel and $\mathrm{CO}_{2}$ price levels and an increase of $\mathrm{CO}_{2}$ and coal price volatility seems possible in the near to mid future. One reason are the ambitious EU emission reduction targets and another is the increasing correlation of world market prices for coal with highly volatile oil prices. This could improve the relative riskiness of the CCGT technology compared to coal. To assess the impact of such a scenario, we assume the standard deviation of the coal operating costs to be increased by $50 \%$. As the mean operating costs are assumed to remain constant, the variance increase implies also changed conditional distribution parameters for both technologies. Furthermore, the (unconditional) variance of the difference time series decreases, resulting in 

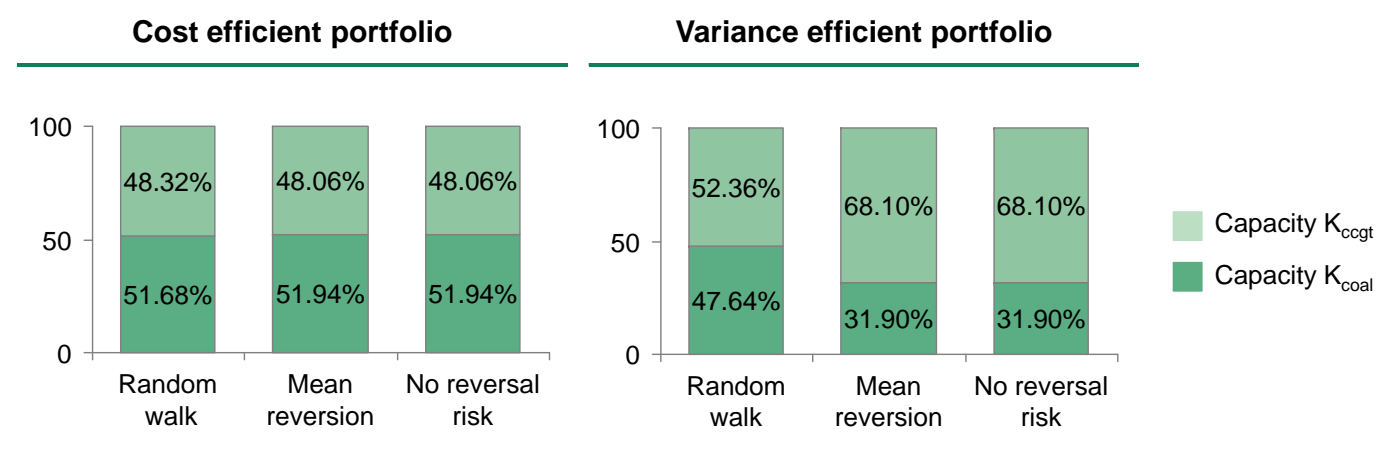

Figure 12: Capacity structure (as a percentage of maximum demand $D(0)$ ) of purely cost and variance efficient generation portfolios consisting of new CCGT and hard coal technologies under the random walk and mean reversion hypothesis and for the approximating calculation with neglected reversal risk. The standard deviation of the coal technology is increased by $50 \%$ compared to the empirically estimated values from Tab. 3.

a slightly reduced likelihood for reversals in the merit order of $21.4 \%$.

The resulting impact on the structure of the purely cost and the purely variance efficient portfolio is depicted in Fig. 12: While the cost efficient portfolio structure remains widely unchanged, the variance efficient portfolio includes now both technologies with a capacity share of about one third coal and two third gas technology. Notably, there is again a significant discrepancy in the efficient fuel mix based on the calculation with neglected risk of reversals in the merit order and the calculation with a reversal risk under the random walk hypothesis. The calculation with the mean reversion hypothesis again yields results close the case with neglected reversal risk.

We start by assuming again risk-neutrality (i.e. $A=0$ ) to assess the benefit of overcapacities (cf. Fig. 13, upper left): As in the previous section, building overcapacities becomes only efficient for decreased levels of investment costs. Thereby, the required reduction of CCGT investment costs is even higher compared to the previous section with the lower coal variance. This is plausible keeping in mind the necessary condition for overcapacities discussed in Eqn. (33): Since the higher coal variance implies a reduced risk for reversals in the merit order, $\mathbf{P}\left(s_{1}\right)$, the CCGT investment costs must even be smaller to satisfy the left part of condition (33).

With both technologies being included in the cost and in the variance efficient portfolio, it could intuitively be expected that with increasing risk aversion building overcapacities becomes more efficient. However, it can still be observed that with increasing societal risk aversion overcapacities become less attractive (cf. Fig. 13). This at first sight surprising result is due to the fact that the variance term in the objective function is not only driven by the specific variance but also by the expected energy produced by each technology which is subject to the second stage of the optimization problem. To determine the expected value of the produced energy, we consider - as in the classic peak load pricing theory - at the second stage of the optimization a technology dispatch based on the merit order of generation costs: The technology 

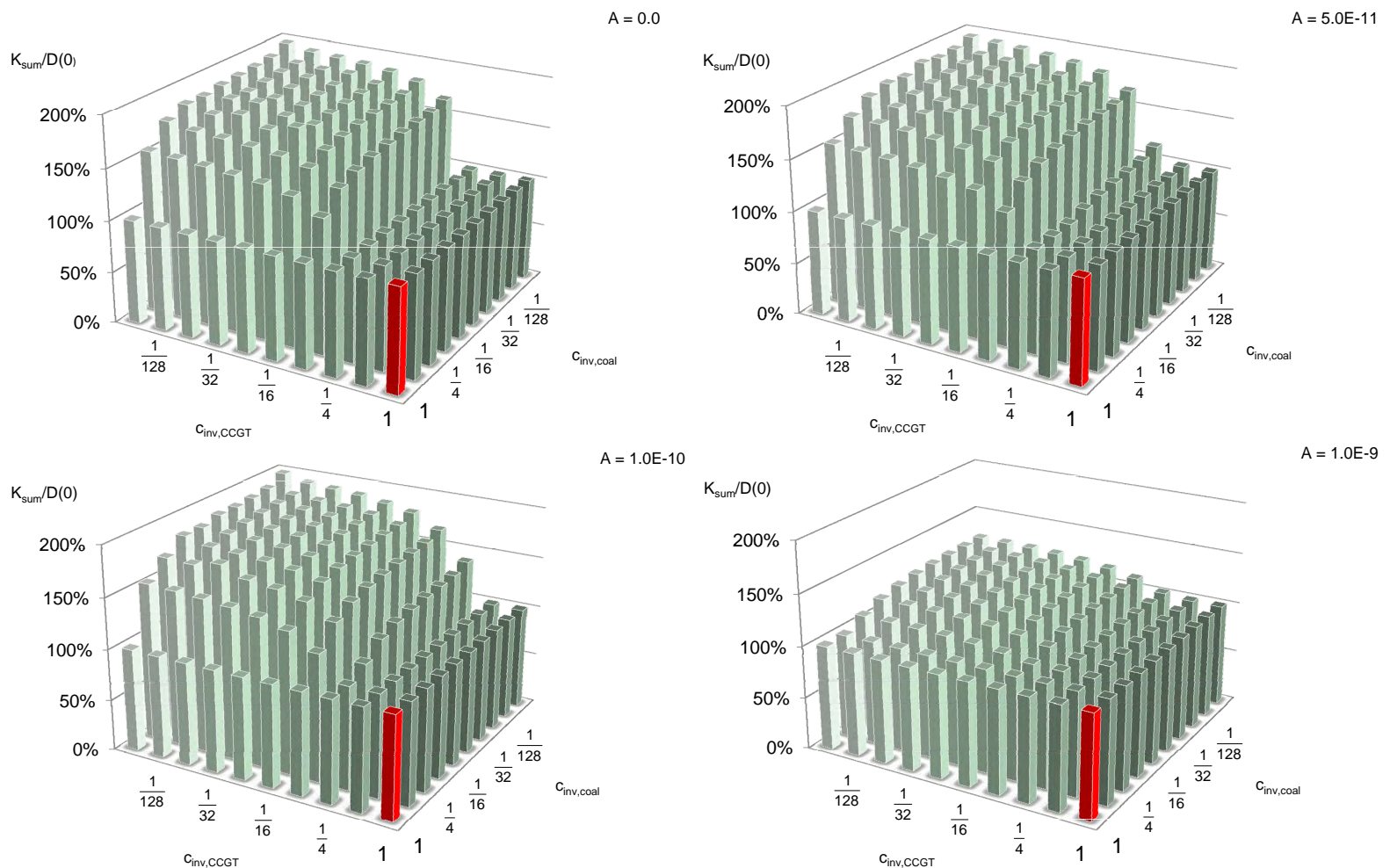

Figure 13: System capacity ratio $\left(K_{\text {coal }}^{*}+K_{C C G T}^{*}\right) / D(0)$ for varying specific investment costs of coal and CCGT technologies at risk aversion levels $A=0$ (upper left), $A=5.0 \cdot 10^{-11}$ (upper right), $A=1.0 \cdot 10^{-10}$ (lower left), and $A=1.0 \cdot 10^{-9}$ (lower right). Differences of operating costs are assumed to follow a random walk. The red stack represents the efficient capacity for the empirically derived annual investment costs. The standard deviation of the coal technology is increased by $50 \%$ compared to the empirically estimated values from Tab. 3.

with the lowest operating costs is used as base load technology, the other to serve peak load demand. This cost-based - and not variance-based - dispatch order influences the expected energy production for each technology. Installing overcapacities in a plant portfolio can hence increase operating flexibility, but may lead to the situation in which the expected mix of energy produced deviates from the variance optimum. Put differently: The most flexible technology mix which would include overcapacities, does typically not minimize the variance of operating costs.

\section{Concluding remarks}

This article analyzes efficient capacity allocation in electricity systems under uncertainty. Special emphasis is put on the impact of reversals in the merit order due to long-term shifts in fuel prices. In particular, 
technologies with operating costs characterized by little difference in mean, high variance and imperfect correlation are affected by these changes in the merit order. The model approach and the obtained insights are also relevant for investment decisions in other industries where different technologies are to be selected to supply an (expected) demand pattern, such as e.g. in transportation applications.

Our results show that risk in (variable) operating cost - measured by its variance - can heavily affect efficient capacity allocation among different technologies. Thereby, two levers of impact can be distinguished:

- Firstly, the cost risk affects the optimal capacity mix given a stable merit order of variable production costs and a firm order of dispatch. However, the efficient technology mix deviates from the purely mean-based optimum only under risk-averse social preferences.

- Secondly, fuel price fluctuations may result in reversals in the merit order which may significantly influence the efficient technology mix. This risk is of particular importance if the considered technologies are characterized by only small differences in their mean costs and exhibit high, uncorrelated cost variances.

In a model application with CCGT and hard coal technologies in the German market environment, we find that a cost efficient portfolio includes a balanced mix oh both technologies. In contrast, only coal is the risk efficient technology given the variance of operating costs based on historical long-term estimates.

Thereby, the characteristic of the underlying difference time series in operating costs in the considered technologies remains a crucial assumption of the model. Assuming random walk versus mean reverting properties of the difference time series may result in significantly different likelihoods for reversals in the merit order and therewith impacts massively the efficient technology mix. Further research may be necessary to provide empirical evidence on the question of the adequate time series model.

With an increasing risk of reversals in the merit order, overcapacities exceeding maximum demand may become economically favorable if investment costs are sufficiently low compared to the expected difference in operating costs. Hence, under risk-neutral preferences with the sole objective to minimize expected generation costs, an increasing risk of reversals in the merit order increases the degree of diversification in efficient technology portfolios. However, our example shows that at current investment costs and historically estimated cost variances, overcapacities are inefficient for CCGT and coal technology portfolios.

The benefits of diversification may change in a risk-averse investment environment where the total variance of operating costs is sought to be reduced: They are reduced if one technology dominates the other(s) with respect to the specific cost risk induced by this technology. Then increasing levels of risk aversion can even lead to efficient technology portfolios which are fully non-diversified and consist of only one technology. In this case, there is also no economic benefit from installing overcapacities - even if these are without additional costs. Hence, diversification of the generation portfolio is - even under risk aversion - not beneficial per-se. 


\section{Appendix A. Symbols and model notation}

\section{Indices}

$u$

$s_{i}$

Plant technology

Merit order state

hours Intra-period time step during analysis period $[0 ; T]$

years Period time step during considered plant lifetime $[0 ; \hat{\tau}]$

\section{Operators}

$\operatorname{Var}[\cdot]$

$\operatorname{Var}\left[\cdot \mid s_{i}\right]$

$\mathbf{E}[\cdot]$

$\mathbf{E}\left[\cdot \mid s_{i}\right]$

$\mathbf{P}(\cdot)$
Variance operator

Conditional variance operator given scenario $s_{i}$

Expectation operator

Conditional expectation operator given scenario $s_{i}$

Probability measure 


\section{Parameters and variables}

\begin{tabular}{|c|c|c|}
\hline$A$ & $1 / €$ & Social risk attitude \\
\hline$D_{t}$ & MW & Total system demand at time $t$ \\
\hline$t_{u}$ & hours & $\begin{array}{l}\text { Minimal operating duration of } u \text { when } \\
\text { representing the base technology }\end{array}$ \\
\hline$O_{u}$ & hours & Minimal operating duration of technology $u$ \\
\hline$p_{u, t}$ & $€ / \mathrm{MWh}_{t h}$ & Fuel price of technology $u$ in period $t$ \\
\hline$\eta_{u}$ & $\mathrm{MWh}_{e} / \mathrm{MWh}_{t h}$ & thermal efficiency of plant technology $u$ \\
\hline$h_{u}$ & $\mathrm{MWh}_{t h} / \mathrm{MWh}_{e}$ & heat rate of plant technology $u$ \\
\hline$e_{u}$ & $\mathrm{tCO}_{2} / \mathrm{MWh}_{t h}$ & emission rate of plant technology $u$ \\
\hline$K_{u}$ & MW & Installed capacity of plant technology $u$ \\
\hline$Q_{u}$ & MWh & Energy produced of plant technology $u$ in period $[0 ; T]$ \\
\hline$Q_{E}$ & $\mathrm{MWh}$ & Total energy produced in the system in period $[0 ; T]$ \\
\hline$y_{u, t}$ & MW & Output level of plant $u$ at time $t$ \\
\hline$C_{i n v, u}$ & $€$ & Annuity of overnight costs (total investment costs) of plant $u$ \\
\hline$c_{i n v, u}$ & $€ / \mathrm{MW}_{e}$ & Annuity of specific overnight costs of plant $u$ per capacity $K_{u}$ \\
\hline$C_{u, t}$ & $€$ & Operating costs of plant $u$ in period $t$ \\
\hline$c_{u, t}$ & $€ / \mathrm{MWh}_{e}$ & Specific operating costs of plant $u$ in period $t$ per output $y_{u, t}$ \\
\hline $\bar{c}_{u}$ & $€ / \mathrm{MWh}_{e}$ & Mean operating costs of plant $u$ \\
\hline$\sigma_{u}$ & $€ / \mathrm{MWh}_{e}$ & Standard deviation of operating costs of plant $u$ \\
\hline$\sigma_{u v}$ & $€^{2} / \mathrm{MWh}_{e}^{2}$ & Covariance of operating costs of plant $u$ and $v$ \\
\hline $\bar{c}_{u \mid s_{i}}$ & $€ / \mathrm{MWh}_{e}$ & Conditional mean operating costs o given scenario $s_{i}$ \\
\hline$\sigma_{u \mid s_{i}}$ & $€ / \mathrm{MWh}_{e}$ & Conditional standard deviation of op. costs given scenario $s_{i}$ \\
\hline$\sigma_{u v \mid s_{i}}$ & $€^{2} / \mathrm{MWh}_{e}^{2}$ & Conditional covariance of op. costs given scenario $s_{i}$ \\
\hline$\rho$ & - & Coefficient of correlation \\
\hline
\end{tabular}




\section{Appendix B. Mathematical Appendix}

\section{Appendix B.1. Calculation of conditional expectations and variances}

To calculate the conditional expectations and variances used in the optimization problem from Eqs. (9)-(12), we start with the conditional joint distribution of $\tilde{c}_{1}, \tilde{c}_{2}$ given $\tilde{c}_{1} \leq \tilde{c}_{2}$ which can be obtained as the truncated distribution (see Figure B.14) with density

$$
f_{1,2}\left(c_{1}, c_{2} \mid \tilde{s}=s_{0}\right)=f_{1,2}\left(c_{1}, c_{2} \mid c_{1}<c_{2}\right)=\frac{\varphi_{1,2}\left(c_{1}, c_{2}, \rho\right)}{\mathbf{P}\left(\tilde{s}=s_{0}\right)}, \text { for }-\infty<c_{1} \leq c_{2}<\infty,
$$

where $\mathbf{P}\left(\tilde{s}=s_{0}\right)$ denotes the fuel-switch likelihood which can be computed from the distribution of differences in operating costs $\tilde{z}=\tilde{c}_{2}-\tilde{c}_{1}$ as discussed in Eqn. (51). Next, the conditional densities of $\tilde{c}_{1}$, $\tilde{c}_{2}$ under the condition $\tilde{c}_{1} \leq \tilde{c}_{2}$ (see Figure B.15) and given a fixed value of $c_{2}, c_{1}$, respectively, are determined as:

$$
\begin{aligned}
& f_{1}\left(c_{1} \mid c_{1} \leq c_{2}\right)=\frac{f_{1,2}\left(c_{1}, c_{2} \mid c_{1} \leq c_{2}\right)}{\varphi_{2}\left(c_{2}\right)}=\frac{\varphi_{1,2}\left(c_{1}, c_{2}, \rho\right)}{\Phi(0) \varphi_{2}\left(c_{2}\right)} \\
& f_{2}\left(c_{2} \mid c_{1} \leq c_{2}\right)=\frac{f_{1,2}\left(c_{1}, c_{2} \mid c_{1} \leq c_{2}\right)}{\varphi_{1}\left(c_{1}\right)}=\frac{\varphi_{1,2}\left(c_{1}, c_{2}, \rho\right)}{\Phi(0) \varphi_{1}\left(c_{1}\right)}
\end{aligned}
$$

From these, we can straightforwardly derive the (single) conditional expectations as

$$
\begin{aligned}
& \mathbf{E}\left[\tilde{c}_{1} \mid c_{2} \wedge\left(c_{1} \leq c_{2}\right)\right]=\int_{-\infty}^{c_{2}} c_{1} f_{1}\left(c_{1} \mid c_{1} \leq c_{2}\right) \mathrm{d} c_{1}=\int_{-\infty}^{c_{2}} c_{1} \frac{\varphi_{1,2}\left(c_{1}, c_{2}, \rho\right)}{\Phi(0) \varphi_{2}\left(c_{2}\right)} \mathrm{d} c_{1} \\
& \mathbf{E}\left[\tilde{c}_{1} \mid c_{2} \wedge\left(c_{1}>c_{2}\right)\right]=\int_{c_{2}}^{\infty} c_{1} f_{1}\left(c_{1} \mid c_{1}>c_{2}\right) \mathrm{d} c_{1}=\int_{c_{2}}^{\infty} c_{1} \frac{\varphi_{1,2}\left(c_{1}, c_{2}, \rho\right)}{(1-\Phi(0)) \varphi_{2}\left(c_{2}\right)} \mathrm{d} c_{1} \\
& \mathbf{E}\left[\tilde{c}_{2} \mid c_{1} \wedge\left(c_{1} \leq c_{2}\right)\right]=\int_{c_{1}}^{\infty} c_{2} f_{2}\left(c_{2} \mid c_{1} \leq c_{2}\right) \mathrm{d} c_{2}=\int_{c_{1}}^{\infty} c_{2} \frac{\varphi_{1,2}\left(c_{1}, c_{2}, \rho\right)}{\Phi(0) \varphi_{1}\left(c_{1}\right)} \mathrm{d} c_{2} \\
& \mathbf{E}\left[\tilde{c}_{2} \mid c_{1} \wedge\left(c_{1}>c_{2}\right)\right]=\int_{-\infty}^{c_{1}} c_{2} f_{2}\left(c_{2} \mid c_{1}>c_{2}\right) \mathrm{d} c_{2}=\int_{-\infty}^{c_{1}} c_{2} \frac{\varphi_{1,2}\left(c_{1}, c_{2}, \rho\right)}{(1-\Phi(0)) \varphi_{1}\left(c_{1}\right)} \mathrm{d} c_{2}
\end{aligned}
$$

Here, conditional expectations $\mathbf{E}\left[\tilde{c}_{1} \mid c_{2} \wedge\left(c_{1} \lesseqgtr c_{2}\right)\right]=g\left(c_{2}\right)$ and $\mathbf{E}\left[\tilde{c}_{2} \mid c_{1} \wedge\left(c_{1} \lesseqgtr c_{2}\right)\right]=g\left(c_{1}\right)$ represent functions which are solely dependent on $c_{1}$ and $c_{2}$, respectively. Hence it makes sense to define the conditional expectation $\bar{c}_{u \mid s_{0}} \equiv \mathbf{E}\left[\tilde{c}_{u} \mid \tilde{s}=s_{0}\right]$ given the default fuel cost order scenario $s_{0}$ as the double expectation $\mathbf{E}\left[\mathbf{E}\left[\tilde{c}_{1} \mid c_{2} \wedge\left(c_{1} \leq c_{2}\right)\right]\right]$. Straightforwardly, we obtain

$$
\begin{aligned}
& \mathbf{E}\left[\tilde{c}_{1} \mid \tilde{s}=s_{0}\right]:=\mathbf{E}\left[\mathbf{E}\left[\tilde{c}_{1} \mid c_{2} \wedge\left(c_{1} \leq c_{2}\right)\right]\right]=\int_{-\infty}^{\infty} \int_{-\infty}^{c_{2}} c_{1} \frac{\varphi_{1,2}\left(c_{1}, c_{2}, \rho\right)}{\Phi(0) \varphi_{2}\left(c_{2}\right)} \mathrm{d} c_{1} \mathrm{~d} c_{2} \\
& \mathbf{E}\left[\tilde{c}_{1} \mid \tilde{s}=s_{1}\right]:=\mathbf{E}\left[\mathbf{E}\left[\tilde{c}_{1} \mid c_{2} \wedge\left(c_{1}>c_{2}\right)\right]\right]=\int_{-\infty}^{\infty} \int_{c_{2}}^{\infty} c_{1} \frac{\varphi_{1,2}\left(c_{1}, c_{2}, \rho\right)}{(1-\Phi(0)) \varphi_{2}\left(c_{2}\right)} \mathrm{d} c_{1} \mathrm{~d} c_{2} \\
& \mathbf{E}\left[\tilde{c}_{2} \mid \tilde{s}=s_{0}\right]:=\mathbf{E}\left[\mathbf{E}\left[\tilde{c}_{2} \mid c_{1} \wedge\left(c_{1} \leq c_{2}\right)\right]\right]=\int_{-\infty}^{\infty} \int_{c_{1}}^{\infty} c_{2} \frac{\varphi_{1,2}\left(c_{1}, c_{2}, \rho\right)}{\Phi(0) \varphi_{1}\left(c_{1}\right)} \mathrm{d} c_{2} \mathrm{~d} c_{1} \\
& \mathbf{E}\left[\tilde{c}_{2} \mid \tilde{s}=s_{1}\right]:=\mathbf{E}\left[\mathbf{E}\left[\tilde{c}_{2} \mid c_{1} \wedge\left(c_{1}>c_{2}\right)\right]\right]=\int_{-\infty}^{\infty} \int_{-\infty}^{c_{1}} c_{2} \frac{\varphi_{1,2}\left(c_{1}, c_{2}, \rho\right)}{(1-\Phi(0)) \varphi_{1}\left(c_{1}\right)} \mathrm{d} c_{2} \mathrm{~d} c_{1}
\end{aligned}
$$

Finally, it is worthwhile to note that $\mathbf{E}\left[\mathbf{E}\left[\tilde{c}_{u} \mid \tilde{s}\right]\right]=\mathbf{E}\left[c_{u}\right]$. Thus, the probability-weighted sum of the conditional expectations of operating costs $\mathbf{E}\left[\tilde{c}_{u} \mid \tilde{s}=s_{i}\right]$ given both fuel cost scenarios, equals the unconditional 
expectation of $\tilde{c}_{u}$, i.e.

$$
\mathbf{E}\left[\mathbf{E}\left[\tilde{c}_{u} \mid \tilde{s}=s_{i}\right]\right]=\sum_{i=1}^{2}\left(\mathbf{P}\left(\tilde{s}=s_{i}\right) \mathbf{E}\left[\tilde{c}_{u} \mid \tilde{s}=s_{i}\right]\right)=\mathbf{E}\left[\tilde{c}_{u}\right]
$$

Recall that the conditional variance of a random variable $\tilde{x}$ given $\tilde{y}$ is defined as

$$
\operatorname{Var}[\tilde{x} \mid \tilde{y}]:=\mathbf{E}\left[(\tilde{x}-\mathbf{E}[\tilde{x} \mid \tilde{y}])^{2} \mid y\right]
$$

In analogy to the conditional expectation, we denote the conditional variance given the fuel price scenario $s_{0}$

$$
\begin{aligned}
& \sigma_{u \mid s_{0}}^{2} \equiv \operatorname{Var}\left[\tilde{c}_{u} \mid \tilde{s}=s_{0}\right]:=\mathbf{E}\left[\operatorname{Var}\left[\tilde{c}_{u} \mid c_{2} \wedge\left(c_{1} \leq c_{2}\right)\right]\right]=\mathbf{E}\left[\mathbf{E}\left[\tilde{c}_{u}^{2} \mid c_{2} \wedge\left(c_{1} \leq c_{2}\right)\right]\right]-\mathbf{E}\left[\tilde{c}_{u} \mid s_{0}\right]^{2} \\
& \sigma_{u \mid s_{1}}^{2} \equiv \operatorname{Var}\left[\tilde{c}_{u} \mid \tilde{s}=s_{1}\right]:=\mathbf{E}\left[\operatorname{Var}\left[\tilde{c}_{u} \mid c_{2} \wedge\left(c_{1}>c_{2}\right)\right]\right]=\mathbf{E}\left[\mathbf{E}\left[\tilde{c}_{u}^{2} \mid c_{2} \wedge\left(c_{1}>c_{2}\right)\right]\right]-\mathbf{E}\left[\tilde{c}_{u} \mid s_{1}\right]^{2}
\end{aligned}
$$

It can be obtained by incremental computation from the square expectations ${ }^{12}$ with

$$
\begin{aligned}
& \operatorname{Var}\left[\tilde{c}_{1} \mid \tilde{s}=s_{0}\right]=\int_{-\infty}^{\infty} \int_{-\infty}^{c_{2}} c_{1}^{2} \frac{\varphi_{1,2}\left(c_{1}, c_{2}, \rho\right)}{\Phi(0) \varphi_{2}\left(c_{2}\right)} \mathrm{d} c_{1} \mathrm{~d} c_{2}-\left(\mathbf{E}\left[c_{1} \mid \tilde{s}=s_{0}\right]\right)^{2} \\
& \operatorname{Var}\left[\tilde{c}_{1} \mid \tilde{s}=s_{1}\right]=\int_{-\infty}^{\infty} \int_{c_{2}}^{\infty} c_{1}^{2} \frac{\varphi_{1,2}\left(c_{1}, c_{2}, \rho\right)}{(1-\Phi(0)) \varphi_{2}\left(c_{2}\right)} \mathrm{d} c_{1} \mathrm{~d} c_{2}-\left(\mathbf{E}\left[c_{1} \mid \tilde{s}=s_{1}\right]\right)^{2} \\
& \operatorname{Var}\left[\tilde{c}_{2} \mid \tilde{s}=s_{0}\right]=\int_{-\infty}^{\infty} \int_{c_{1}}^{\infty} c_{2}^{2} \frac{\varphi_{1,2}\left(c_{1}, c_{2}, \rho\right)}{\Phi(0) \varphi_{1}\left(c_{1}\right)} \mathrm{d} c_{2} \mathrm{~d} c_{1}-\left(\mathbf{E}\left[c_{2} \mid \tilde{s}=s_{0}\right]\right)^{2} \\
& \operatorname{Var}\left[\tilde{c}_{2} \mid \tilde{s}=s_{1}\right]=\int_{-\infty}^{\infty} \int_{-\infty}^{c_{1}} c_{2}^{2} \frac{\varphi_{1,2}\left(c_{1}, c_{2}, \rho\right)}{(1-\Phi(0)) \varphi_{1}\left(c_{1}\right)} \mathrm{d} c_{2} \mathrm{~d} c_{1}-\left(\mathbf{E}\left[c_{2} \mid \tilde{s}=s_{1}\right]\right)^{2}
\end{aligned}
$$

In a straightforward manner we obtain for the conditional covariance $\sigma_{12 \mid s_{i}} \equiv \operatorname{Cov}\left[\tilde{c}_{1}, \tilde{c}_{2} \mid \tilde{s}=s_{0}\right]$

$$
\begin{aligned}
\operatorname{Cov}\left[\tilde{c}_{1}, \tilde{c}_{2} \mid \tilde{s}=s_{0}\right] & =\mathbf{E}\left[\tilde{c}_{1} \tilde{c}_{2} \mid \tilde{s}=s_{0}\right]-\mathbf{E}\left[\tilde{c}_{1} \mid \tilde{s}=s_{0}\right] \cdot \mathbf{E}\left[\tilde{c}_{2} \mid \tilde{s}=s_{0}\right] \\
& =\int_{-\infty}^{\infty} \int_{-\infty}^{c_{2}} c_{1} c_{2} \frac{\varphi_{1,2}\left(c_{1}, c_{2}, \rho\right)}{\Phi(0) \varphi_{2}\left(c_{2}\right)} \mathrm{d} c_{1} \mathrm{~d} c_{2}-\mathbf{E}\left[\tilde{c}_{1} \mid \tilde{s}=s_{0}\right] \mathbf{E}\left[\tilde{c}_{2} \mid \tilde{s}=s_{0}\right] \\
\operatorname{Cov}\left[\tilde{c}_{1}, \tilde{c}_{2} \mid \tilde{s}=s_{1}\right] & =\mathbf{E}\left[\tilde{c}_{1} \tilde{c}_{2} \mid \tilde{s}=s_{1}\right]-\mathbf{E}\left[\tilde{c}_{1} \mid \tilde{s}=s_{1}\right] \cdot \mathbf{E}\left[\tilde{c}_{2} \mid \tilde{s}=s_{1}\right] \\
& =\int_{-\infty}^{\infty} \int_{c_{2}}^{\infty} c_{1} c_{2} \frac{\varphi_{1,2}\left(c_{1}, c_{2}, \rho\right)}{(1-\Phi(0)) \varphi_{2}\left(c_{2}\right)} \mathrm{d} c_{1} \mathrm{~d} c_{2}-\mathbf{E}\left[\tilde{c}_{1} \mid \tilde{s}=s_{1}\right] \mathbf{E}\left[\tilde{c}_{2} \mid \tilde{s}=s_{1}\right]
\end{aligned}
$$

\footnotetext{
${ }^{12}$ Alternatively, the expected conditional variance could be derived from the law of total variance, i.e. $\operatorname{Var}[\tilde{x}]=\operatorname{Var}[\tilde{x} \mid \tilde{y}]+$ $\operatorname{Var}[\mathbf{E}[\tilde{x} \mid \tilde{y}]]$. Based on the latter, the expected conditional variance can be written as

$$
\mathbf{E}[\operatorname{Var}[\tilde{x} \mid \tilde{y}]]=\operatorname{Var}[\tilde{x}]-\mathbf{E}[\operatorname{Var}[\mathbf{E}[\tilde{x} \mid \tilde{y}]]]
$$
}



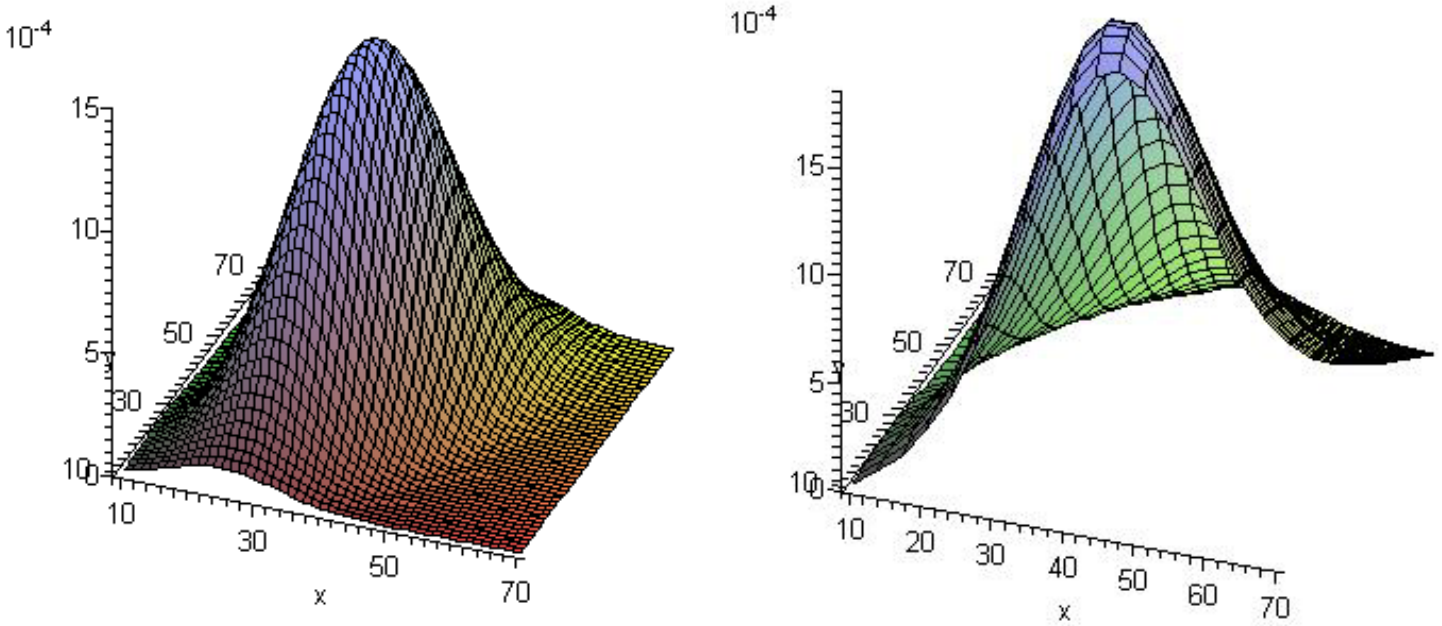

Figure B.14: Unconditional bivariate density function $\varphi_{1,2}\left(c_{1}, c_{2}, \rho\right)$ (left) and conditional (truncated) bivariate density function $f_{1,2}\left(c_{1}, c_{2} \mid c_{1}<c_{2}\right)$ (right).

$10^{-4}$
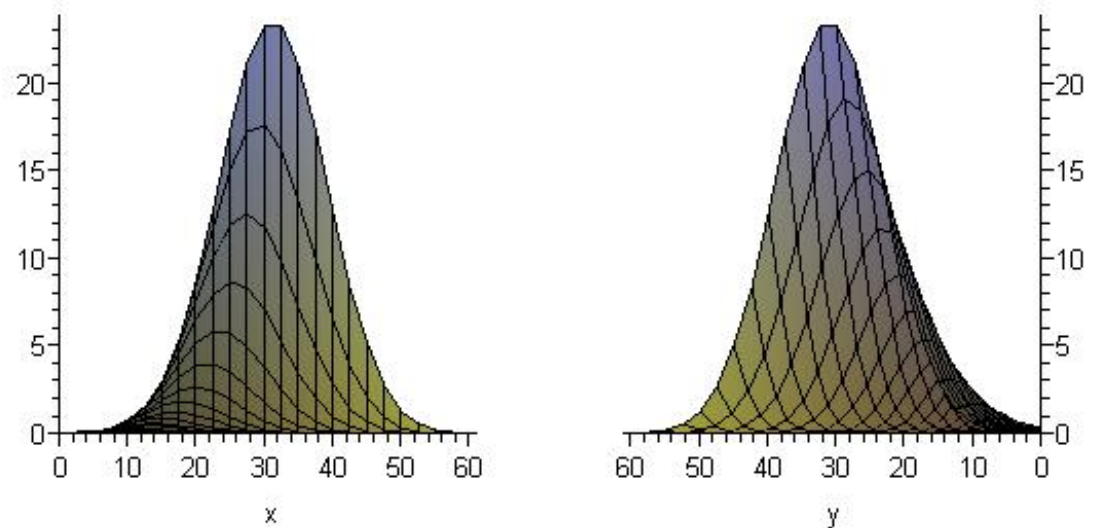

Figure B.15: Marginal densities $f_{1}\left(c_{1}, c_{2} \mid c_{1}<c_{2}\right)$ (left) and $f_{2}\left(c_{1}, c_{2} \mid c_{1}<c_{2}\right)$ (right). 
Appendix B.2. The risk-adjusted portfolio problem with risk of reversals in the merit order

Proof of Eqn. (10). Total expected generation costs can be calculated as

$$
\begin{aligned}
\mathbf{E}\left[C_{o p}\right] & =\mathbf{E}\left[\mathbf{E}\left[C_{o p} \mid \tilde{s}\right]\right]=\mathbf{E}\left[\mathbf{E}\left[\sum_{u}\left(Q_{u \mid \tilde{s}} \tilde{c}_{u}\right) \mid \tilde{s}\right]\right]=\mathbf{E}\left[\sum_{u} \mathbf{E}\left[\left(Q_{u \mid \tilde{s}} \tilde{c}_{u}\right) \mid \tilde{s}\right]\right] \\
& =\mathbf{E}\left[\sum_{u} Q_{u \mid \tilde{s}} \mathbf{E}\left[\tilde{c}_{u} \mid \tilde{s}\right]\right]=\sum_{u} \mathbf{E}\left[Q_{u \mid \tilde{s}} \mathbf{E}\left[\tilde{c}_{u} \mid \tilde{s}\right]\right]=\sum_{u} \sum_{i}\left(\mathbf{P}\left(\tilde{s}=s_{i}\right) Q_{u \mid s_{i}} \mathbf{E}\left[\tilde{c}_{u} \mid \tilde{s}=s_{i}\right]\right)
\end{aligned}
$$

Similarly, conditional variance is used to calculate total variance of generation costs. Thereby, the total variance consists of intra-scenario variance and inter-scenario variance. For the variance of generation costs of technology $u$ we obtain by inserting the conditional expectation as calculated above followed by rewriting

$$
\begin{aligned}
\operatorname{Var}\left[C_{u}\right] & =\mathbf{E}\left[\mathbf{E}\left[\left(Q_{u \mid \tilde{s}}\right)^{2} \tilde{c}_{u}^{2} \mid \tilde{s}\right]\right]-\left(\mathbf{E}\left[\mathbf{E}\left[Q_{u \mid \tilde{s}} \tilde{c}_{u} \mid \tilde{s}\right]\right]\right)^{2} \\
& =\mathbf{E}\left[\mathbf{E}\left[\left(Q_{u \mid \tilde{s}}\right)^{2} \tilde{c}_{u}^{2} \mid \tilde{s}\right]\right]-\mathbf{E}\left[\left(\mathbf{E}\left[Q_{u \mid \tilde{s}} \tilde{c}_{u} \mid \tilde{s}\right]\right)^{2}\right]+\mathbf{E}\left[\left(Q_{u \mid \tilde{s}} \mathbf{E}\left[\tilde{c}_{u} \mid \tilde{s}\right]\right)^{2}\right]-\left(\mathbf{E}\left[Q_{u \mid \tilde{s}} \mathbf{E}\left[\tilde{c}_{u} \mid \tilde{s}\right]\right]\right)^{2} \\
& =\mathbf{E}\left[\mathbf{E}\left[\left(Q_{u \mid \tilde{s}}\right)^{2} \tilde{c}_{u}^{2} \mid \tilde{s}\right]-\left(\mathbf{E}\left[Q_{u \mid \tilde{s}} \tilde{c}_{u} \mid \tilde{s}\right]\right)^{2}\right]+\mathbf{E}\left[\left(Q_{u \mid \tilde{s}} \mathbf{E}\left[\tilde{c}_{u} \mid \tilde{s}\right]\right)^{2}\right]-\left(\mathbf{E}\left[Q_{u \mid \tilde{s}} \mathbf{E}\left[\tilde{c}_{u} \mid \tilde{s}\right]\right]\right)^{2} \\
& =\mathbf{E}\left[\operatorname{Var}\left[Q_{u \mid} \tilde{c}_{u} \mid \tilde{s}\right]\right]+\operatorname{Var}\left[Q_{u \mid \tilde{s}} \mathbf{E}\left[\tilde{c}_{u} \mid \tilde{s}\right]\right] \\
& =\mathbf{E}\left[\left(Q_{u \mid \tilde{s}}\right)^{2} \cdot \operatorname{Var}\left[\tilde{c}_{u} \mid \tilde{s}\right]\right]+\mathbf{E}\left[\left(Q_{u \mid \tilde{s}} \mathbf{E}\left[\tilde{c}_{u} \mid \tilde{s}\right]\right)^{2}\right]-\left(\mathbf{E}\left[Q_{u \mid \tilde{s}} \mathbf{E}\left[\tilde{c}_{u} \mid \tilde{s}\right]\right]\right)^{2} \\
& =\mathbf{E}\left[\left(Q_{u \mid \tilde{s}}\right)^{2} \cdot\left(\operatorname{Var}\left[\tilde{c}_{u} \mid \tilde{s}\right]+\left(\mathbf{E}\left[\tilde{c}_{u} \mid \tilde{s}\right]\right)^{2}\right)\right]-\left(\mathbf{E}\left[Q_{u \mid \tilde{s}} \mathbf{E}\left[\tilde{c}_{u} \mid \tilde{s}\right]\right]\right)^{2}
\end{aligned}
$$

Similarly, the covariance of operating costs of technologies $u, v$ can be derived as

$$
\begin{aligned}
\operatorname{Cov}\left[C_{u}, C_{v}\right]= & \mathbf{E}\left[\mathbf{E}\left[\left(Q_{u \mid \tilde{s}}\right) \tilde{c}_{u} \mid \tilde{s}\right] \cdot \mathbf{E}\left[\left(Q_{v \mid \tilde{s}}\right) \tilde{c}_{v} \mid \tilde{s}\right]\right]-\mathbf{E}\left[\mathbf{E}\left[Q_{u \mid \tilde{s}} \tilde{c}_{u} \mid \tilde{s}\right]\right] \cdot \mathbf{E}\left[\mathbf{E}\left[Q_{v \mid \tilde{s}} \tilde{c}_{v} \mid \tilde{s}\right]\right] \\
= & \mathbf{E}\left[Q_{u \mid \tilde{s}} Q_{v \mid \tilde{s}} \operatorname{Cov}\left[\tilde{c}_{u}, \tilde{c}_{v} \mid \tilde{s}\right]\right]+\mathbf{E}\left[Q_{u \mid \tilde{s}} \mathbf{E}\left[\tilde{c}_{u} \mid \tilde{s}\right] Q_{v \mid \tilde{s}} \mathbf{E}\left[\tilde{c}_{v} \mid \tilde{s}\right]\right] \\
& -\mathbf{E}\left[Q_{u \mid \tilde{s}} \mathbf{E}\left[\tilde{c}_{u} \mid \tilde{s}\right]\right] \mathbf{E}\left[Q_{v \mid \tilde{s}} \mathbf{E}\left[\tilde{c}_{v} \mid \tilde{s}\right]\right] \\
= & \mathbf{E}\left[Q_{u \mid \tilde{s}} Q_{v \mid \tilde{s}}\left(\operatorname{Cov}\left[\tilde{c}_{u}, \tilde{c}_{v} \mid \tilde{s}\right]+\mathbf{E}\left[\tilde{c}_{u} \mid \tilde{s}\right] \mathbf{E}\left[\tilde{c}_{v} \mid \tilde{s}\right]\right)\right] \\
& -\mathbf{E}\left[Q_{u \mid s} \mathbf{E}\left[\tilde{c}_{u} \mid \tilde{s}\right]\right] \mathbf{E}\left[Q_{v \mid s} \mathbf{E}\left[\tilde{c}_{v} \mid \tilde{s}\right]\right]
\end{aligned}
$$

Hence, the total variance of operating costs for the set of all technologies $u=\{1, \ldots, n\}$ can be calculated as:

$$
\operatorname{Var}\left[C_{o p}\right]=\operatorname{Var}\left[\sum_{u} C_{u}\right]=\sum_{u} \operatorname{Var}\left[C_{u}\right]+\sum_{u} \sum_{v, v \neq u} \operatorname{Cov}\left[C_{u}, C_{v}\right] .
$$

By inserting $\mathbf{E}\left[C_{o p}\right]$ and $\operatorname{Var}\left[C_{o p}\right]$ as derived above into Eqn. (5), we obtain as objective function of the 
optimization problem as formulated in Eqs. (9)-(12):

$$
\begin{aligned}
L= & \sum_{u}\left(K_{u} c_{i n v, u}+\mathbf{E}\left[Q_{u \mid \tilde{s}} \mathbf{E}\left[\tilde{c}_{u} \mid \tilde{s}\right]\right]\right) \\
& +\frac{A}{2} \sum_{u} \sum_{v}\left(\mathbf{E}\left[Q_{u \mid \tilde{s}} Q_{v \mid \tilde{s}}\left(\operatorname{Cov}\left[\tilde{c}_{u}, \tilde{c}_{v} \mid \tilde{s}\right]+\mathbf{E}\left[\tilde{c}_{u} \mid \tilde{s}\right] \cdot \mathbf{E}\left[\tilde{c}_{v} \mid \tilde{s}\right]\right)\right]-\mathbf{E}\left[Q_{u \mid \tilde{s}} \mathbf{E}\left[\tilde{c}_{u} \mid \tilde{s}\right]\right] \cdot \mathbf{E}\left[Q_{v \mid \tilde{s}} \mathbf{E}\left[\tilde{c}_{v} \mid \tilde{s}\right]\right]\right) \\
= & \sum_{u}\left(K_{u} c_{i n v, u}+\mathbf{E}\left[Q_{u \mid \tilde{s}} \mathbf{E}\left[\tilde{c}_{u} \mid \tilde{s}\right]\right]+\frac{1}{2} A\left(\mathbf{E}\left[\left(Q_{u \mid \tilde{s}}\right)^{2}\left(\operatorname{Var}\left[\tilde{c}_{u} \mid \tilde{s}\right]+\left(\mathbf{E}\left[\tilde{c}_{u} \mid \tilde{s}\right]\right)^{2}\right)\right]-\left(\mathbf{E}\left[Q_{u \mid s} \mathbf{E}\left[\tilde{c}_{u} \mid \tilde{s}\right]\right]\right)^{2}\right.\right. \\
& \left.\left.+\sum_{v, v \neq u}\left(\mathbf{E}\left[Q_{u \mid \tilde{s}} Q_{v \mid \tilde{s}}\left(\operatorname{Cov}\left[\tilde{c}_{u}, \tilde{c}_{v} \mid \tilde{s}\right]+\mathbf{E}\left[\tilde{c}_{u} \mid \tilde{s}\right] \cdot \mathbf{E}\left[\tilde{c}_{v} \mid \tilde{s}\right]\right)\right]-\mathbf{E}\left[Q_{u \mid \tilde{s}} \mathbf{E}\left[\tilde{c}_{u} \mid \tilde{s}\right]\right] \cdot \mathbf{E}\left[Q_{v \mid \tilde{s}} \mathbf{E}\left[\tilde{c}_{v} \mid \tilde{s}\right]\right]\right)\right)\right) \\
= & \sum_{u}\left(K_{u} c_{i n v, u}+\mathbf{E}\left[Q_{u \mid \tilde{s}} \mathbf{E}\left[\tilde{c}_{u} \mid \tilde{s}\right]\right]+\frac{1}{2} A\left(\mathbf{E}\left[\left(Q_{u \mid \tilde{s}}\right)^{2}\left(\operatorname{Var}\left[\tilde{c}_{u} \mid \tilde{s}\right]+\left(\mathbf{E}\left[\tilde{c}_{u} \mid \tilde{s}\right]\right)^{2}\right)\right]-\left(\mathbf{E}\left[Q_{u \mid \tilde{s}} \mathbf{E}\left[\tilde{c}_{u} \mid \tilde{s}\right]\right]\right)^{2}\right)\right) \\
& +A\left(\mathbf{E}\left[Q_{1 \mid \tilde{s}} Q_{2 \mid \tilde{s}}\left(\operatorname{Cov}\left[\tilde{c}_{1}, \tilde{c}_{2} \mid \tilde{s}\right]+\mathbf{E}\left[\tilde{c}_{1} \mid \tilde{s}\right] \cdot \mathbf{E}\left[\tilde{c}_{2} \mid \tilde{s}\right]\right)\right]-\mathbf{E}\left[Q_{1 \mid \tilde{s}} \mathbf{E}\left[\tilde{c}_{1} \mid \tilde{s}\right]\right] \cdot \mathbf{E}\left[Q_{2 \mid \tilde{s}} \mathbf{E}\left[\tilde{c}_{2} \mid \tilde{s}\right]\right]\right) \\
= & \sum_{u}\left(K_{u} c_{i n v, u}+\sum_{i=0}^{1} \mathbf{P}\left(\tilde{s}=s_{i}\right)\left(Q_{u \mid s_{i}} \mathbf{E}\left[\tilde{c}_{u} \mid s_{i}\right]+\frac{1}{2} A \sum_{v} Q_{u \mid s_{i}} Q_{v \mid s_{i}}\left(\operatorname{Cov}\left[\tilde{c}_{u}, \tilde{c}_{v} \mid s_{i}\right]+\mathbf{E}\left[\tilde{c}_{u} \mid s_{i}\right] \mathbf{E}\left[\tilde{c}_{v} \mid s_{i}\right]\right)\right)\right. \\
& \left.\left.+A Q_{1 \mid \tilde{s}} Q_{2 \mid \tilde{s}}\left(\operatorname{Cov}\left[\tilde{c}_{1}, \tilde{c}_{2} \mid \tilde{s}\right]+\mathbf{E}\left[\tilde{c}_{1} \mid \tilde{s}\right] \cdot \mathbf{E}\left[\tilde{c}_{2} \mid \tilde{s}\right]\right)\right)\right) \\
& -\frac{A}{2} \sum_{i=0}^{1} \mathbf{P}\left(\tilde{s}=s_{i}\right)\left(\sum_{u} Q_{u \mid \tilde{s}} \mathbf{E}\left[\tilde{c}_{u} \mid \tilde{s}\right]\right)^{2}+\frac{A}{2}\left(\sum_{i=0}^{1} \mathbf{P}\left(\tilde{s}=s_{i}\right) Q_{u \mid \tilde{s}} \mathbf{E}\left[\tilde{c}_{u} \mid \tilde{s}\right]\right)^{2}
\end{aligned}
$$

Appendix B.3. Standard solutions to the purely cost efficient portfolio with uncertainty in the merit order Proof of Eqs. (29) and (30). In an extensive form, the Lagrangian (28) can be written as

$$
\begin{gathered}
\mathcal{L}_{c}=\sum_{u=1}^{2} K_{u} c_{i n v, u}+Q_{1 \mid s_{0}} \mathbf{P}\left(s_{0}\right) \bar{c}_{1 \mid s_{0}}+Q_{1 \mid s_{1}} \mathbf{P}\left(s_{1}\right) \bar{c}_{1 \mid s_{1}} \\
+Q_{2 \mid s_{0}} \mathbf{P}\left(s_{0}\right) \bar{c}_{2 \mid s_{0}}+Q_{2 \mid s_{1}} \mathbf{P}\left(s_{1}\right) \bar{c}_{2 \mid s_{1}}+\lambda\left(D(0)-K_{1}-K_{2}\right)
\end{gathered}
$$

Denoting $z:=c_{2}-c_{1}$, the KKT-conditions (29) and (30) can be derived from $\mathcal{L}_{c}$ as follows:

$$
\begin{aligned}
\frac{\partial \mathcal{L}_{c}}{\partial K_{1}} & =c_{i n v, 1}-\lambda+\frac{\partial Q_{1 \mid s_{0}}}{\partial K_{1}} \cdot \mathbf{P}\left(s_{0}\right) \bar{c}_{1 \mid s_{0}}+\frac{\partial Q_{2 \mid s_{0}}}{\partial K_{1}} \cdot \mathbf{P}\left(s_{0}\right) \bar{c}_{2 \mid s_{0}} \\
& =c_{i n v, 1}-\lambda+t_{1} \mathbf{P}\left(s_{0}\right) \bar{c}_{1 \mid s_{0}}-t_{1} \mathbf{P}\left(s_{0}\right) \bar{c}_{2 \mid s_{0}}=t_{1} \mathbf{P}\left(s_{0}\right) \cdot \mathbf{E}\left[\tilde{z} \mid s_{0}\right] \\
\frac{\partial \mathcal{L}_{c}}{\partial K_{2}} & =c_{i n v, 2}-\lambda+\frac{\partial Q_{1 \mid s_{1}}}{\partial K_{2}} \cdot \mathbf{P}\left(s_{1}\right) \bar{c}_{1 \mid s_{1}}+\frac{\partial Q_{2 \mid s_{1}}}{\partial K_{2}} \cdot \mathbf{P}\left(s_{1}\right) \bar{c}_{2 \mid s_{1}} \\
& =c_{i n v, 1}-\lambda-t_{2} \mathbf{P}\left(s_{1}\right) \bar{c}_{1 \mid s_{1}}+t_{2} \mathbf{P}\left(s_{1}\right) \bar{c}_{2 \mid s_{1}}=t_{2} \mathbf{P}\left(s_{1}\right) \cdot \mathbf{E}\left[\tilde{z} \mid s_{1}\right]
\end{aligned}
$$

Proposition 2.1. With $z:=c_{2}-c_{1}$ denoting the difference in operating costs of technologies 1 and 2 , the purely cost-minimal portfolio with a risk of reversal in the merit order $\mathbf{P}\left(s_{1}\right)$ consists of technology 2 if and only if

$$
c_{i n v, 1}-c_{i n v, 2} \geq T \mathbf{P}\left(s_{0}\right) \mathbf{E}\left[\tilde{z} \mid s_{0}\right] .
$$


In contrast, the portfolio consists of technology 1 if and only if

$$
c_{i n v, 1}-c_{i n v, 2} \leq T \mathbf{P}\left(s_{1}\right) \mathbf{E}\left[\tilde{z} \mid s_{1}\right]
$$

Proof of Proposition 2.1. We will first prove the equivalence $K_{2}^{*}=0 \Leftrightarrow c_{i n v, 1}-c_{i n v, 2} \leq T \mathbf{P}\left(s_{1}\right)\left(\bar{c}_{2 \mid s_{1}}-\bar{c}_{1 \mid s_{1}}\right)$ by showing the validity of the two implications:

Given $K_{2}^{*}=0$, it can be concluded in the case $\lambda>0$ (i.e. no overcapacities in the optimum) $t_{1}^{*}=0$ since $K_{1}=D(0)=D\left(t_{1}^{*}\right)$ according to Eqn. (31). Since for the lower bound of the operating time of the respective base load technology it holds $D\left(t_{u}^{*}\right)=K_{u}^{*}$, it must furthermore be $t_{2}^{*}=T$. Since Eqn. (29) holds with equality, $\lambda$ can be be eliminated by subtraction of Eqs. ((29) and ((30) yielding

$$
c_{i n v, 1}-c_{i n v, 2} \leq T \mathbf{P}\left(s_{1}\right)\left(\bar{c}_{2 \mid s_{1}}-\bar{c}_{1 \mid s_{1}}\right)
$$

The case $\lambda=0$ (i.e. there may be overcapacities in the optimum) can be rejected with the initial assumption: Since $K_{2}^{*}=0$ implies $t_{1}^{*}=0$, KKT condition (30) yields the contradiction $c_{i n v, 1}=0 .{ }^{13}$

To prove the converse implication, we assume for $c_{i n v, 1}-c_{i n v, 2} \leq T \mathbf{P}\left(s_{1}\right)\left(\bar{c}_{2 \mid s_{1}}-\bar{c}_{1 \mid s_{1}}\right)$ without limitation of the generality $K_{2}^{*}>0$. This implies $0 \leq t_{2}^{*}<T, 0<t_{1}^{*} \leq T$. Hence KKT condition (30) has to be fulfilled with equality. Eliminating $\lambda$ through subtraction of KKT conditions (29) and (30) yields

$$
c_{i n v, 1}-c_{i n v, 2} \geq t_{1}^{*} \mathbf{P}\left(s_{0}\right)\left(\bar{c}_{2 \mid s_{0}}-\bar{c}_{1 \mid s_{0}}\right)+t_{2}^{*} \mathbf{P}\left(s_{1}\right)\left(\bar{c}_{2 \mid s_{1}}-\bar{c}_{1 \mid s_{1}}\right)
$$

However, the latter inequality is contradictory to the initial assumption for all feasible $0 \leq t_{2}^{*}<T, 0<$ $t_{1}^{*} \leq T$ since $\left(\bar{c}_{2 \mid s_{0}}-\bar{c}_{1 \mid s_{0}}\right)>0$ and $\left(\bar{c}_{2 \mid s_{1}}-\bar{c}_{1 \mid s_{1}}\right)<0$. Thus it follows $K_{2}^{*}=0$ from $c_{i n v, 1}-c_{i n v, 2} \leq$ $T \mathbf{P}\left(s_{1}\right)\left(\bar{c}_{2 \mid s_{1}}-\bar{c}_{1 \mid s_{1}}\right)$.

The proof of the analogue equivalence $K_{1}^{*}=0 \Leftrightarrow c_{i n v, 1}-c_{i n v, 2} \geq-T \mathbf{P}\left(s_{0}\right)\left(\bar{c}_{2 \mid s_{0}}-\bar{c}_{1 \mid s_{0}}\right)$ can be obtained in a straightforward manner analogue to this proof.

Appendix B.4. Standard solutions to the purely variance efficient portfolio with uncertainty in the merit order

Proposition 2.2. Under the restriction that total installed generation capacity must match total demand, i.e. $\lambda \neq 0$, the purely variance minimal portfolio with a risk of reversal in the merit order $\mathbf{P}\left(s_{1}\right)>0$ corresponding to optimization problem (9)-(12) consists of both technologies 1 and 2 if

$$
\left(-\frac{\sigma_{12 \mid s_{0}}-\sigma_{1 \mid s_{0}}^{2}}{\mathbf{E}\left[\tilde{z} \mid s_{0}\right] \mathbf{P}\left(s_{1}\right)}>\bar{c}_{1 \mid s_{1}}-\bar{c}_{1 \mid s_{0}}\right) \wedge\left(\bar{c}_{1 \mid s_{1}}-\bar{c}_{1 \mid s_{0}}<\frac{\sigma_{1 \mid s_{1}}^{2}-\sigma_{12 \mid s_{1}}}{\mathbf{E}\left[\tilde{z} \mid s_{1}\right] \mathbf{P}\left(s_{0}\right)}\right)
$$

\footnotetext{
${ }^{13}$ The rejection of the case $\lambda=0$ is intuitively plausible from an economical perspective, since it does not make sense to build overcapacities in the optimum if only one technology is selected.
} 
Proof of Proposition 2.2. Given the initial assumption $\lambda \neq 0, t_{2}$ can be expressed as a function of $t_{1}$ throughout this proof with $t_{2}\left(t_{1}\right)=R\left(D(0)-D\left(t_{1}\right)\right)$. Hence, $t_{1}$ can be used as the only decision variable in the problem since $K_{1}, K_{2}, t_{2}$ are all functions of $t_{1}$. Theoretically, locating the root $\frac{\mathrm{d} \mathcal{L}_{v}\left(t_{1}, t_{2}\left(t_{1}\right)\right)}{\mathrm{d} t_{1}}=0$ would allow to further discuss the considered solution case. ${ }^{14}$ Since an analytical discussion of the latter derivative seems practically impossible, we use an alternative approach in the proof:

For an interior solution with $0<t_{1}^{*}<T$ (and hence $K_{1}^{*}, K_{2}^{*}>0$ ), KKT conditions (38) and (39) have to be satisfied with equality as necessary optimality condition. Eliminating $\lambda$ through subtraction of these conditions yields $\frac{\partial \mathcal{L}_{v}}{\partial K_{1}}-\frac{\partial \mathcal{L}_{v}}{\partial K_{2}}=0$. For $t_{1}>0$, the latter condition may be equivalently transformed by division through $t_{1}$. Substitution of $Q_{2 \mid s_{i}}$ by utilizing the relation $Q_{2 \mid s_{i}}=Q_{E}-Q_{1 \mid s_{i}}, i \in\{1,2\}$, finally yields the equivalent optimality condition $l\left(t_{1}\right)=r\left(t_{1}\right)$ with

$$
\begin{aligned}
l\left(t_{1}\right):= & \mathbf{P}\left(s_{0}\right)\left(\left(Q_{1 \mid s_{0}}\left(\operatorname{Var}\left[\tilde{z} \mid s_{0}\right]+\mathbf{P}\left(s_{1}\right) \mathbf{E}\left[\tilde{z} \mid s_{0}\right]^{2}\right)-\mathbf{P}\left(s_{1}\right) Q_{1 \mid s_{1}} \mathbf{E}\left[\tilde{z} \mid s_{1}\right] \mathbf{E}\left[\tilde{z} \mid s_{0}\right]\right)-\right. \\
& \left.-Q_{E}\left(\sigma_{2 \mid s_{0}}^{2}-\sigma_{12 \mid s_{0}}+\mathbf{P}\left(s_{1}\right)\left(\bar{c}_{2 \mid s_{0}}-\bar{c}_{2 \mid s_{1}}\right) \mathbf{E}\left[\tilde{z} \mid s_{0}\right]\right)\right) \\
r\left(t_{1}\right):= & \mathbf{P}\left(s_{1}\right) \frac{t_{2}}{t_{1}}\left(\mathbf{P}\left(s_{0}\right) \mathbf{E}\left[\tilde{z} \mid s_{0}\right] Q_{1 \mid s_{0}} \mathbf{E}\left[\tilde{z} \mid s_{1}\right]-\left(\operatorname{Var}\left[\tilde{z} \mid s_{1}\right]+\mathbf{P}\left(s_{0}\right) \mathbf{E}\left[\tilde{z} \mid s_{1}\right]^{2}\right) Q_{1 \mid s_{1}}+\right. \\
& \left.+Q_{E}\left(\sigma_{2 \mid s_{1}}^{2}-\sigma_{12 \mid s_{1}}+\mathbf{P}\left(s_{0}\right)\left(\bar{c}_{2 \mid s_{1}}-\bar{c}_{2 \mid s_{0}}\right) \mathbf{E}\left[\tilde{z} \mid s_{1}\right]\right)\right)
\end{aligned}
$$

Thereby, the corresponding boundaries for $l\left(t_{1}\right)$ can straightforwardly be obtained as

$$
\begin{aligned}
& l(0)=\mathbf{P}\left(s_{0}\right) Q_{E}\left(\mathbf{P}\left(s_{1}\right) \mathbf{E}\left[\tilde{z} \mid s_{0}\right]\left(\bar{c}_{1 \mid s_{1}}-\bar{c}_{1 \mid s_{0}}\right)+\sigma_{1 \mid s_{0}}^{2}-\sigma_{12 \mid s_{0}}\right), \\
& l(T)=-\mathbf{P}\left(s_{0}\right) Q_{E}\left(\mathbf{P}\left(s_{1}\right) \mathbf{E}\left[\tilde{z} \mid s_{0}\right]\left(\bar{c}_{2 \mid s_{0}}-\bar{c}_{2 \mid s_{1}}\right)+\sigma_{2 \mid s_{0}}^{2}-\sigma_{12 \mid s_{0}}\right) .
\end{aligned}
$$

By applying the derivatives

$$
\begin{aligned}
\frac{\mathrm{d} t_{2}\left(t_{1}\right)}{\mathrm{d} t_{1}} & =\frac{\mathrm{d}}{\mathrm{d} t_{1}}\left(R\left(D(0)-D\left(t_{1}\right)\right)\right)=-R^{\prime}\left(D(0)-D\left(t_{1}\right)\right) \cdot D^{\prime}\left(t_{1}\right)<0 \quad \forall t_{1} \in[0, T], \\
\frac{\mathrm{d} Q_{1 \mid s_{0}}\left(t_{1}\right)}{\mathrm{d} t_{1}} & =\frac{\mathrm{d}}{\mathrm{d} t_{1}}\left(\int_{0}^{D\left(t_{1}\right)} R(\kappa) \mathrm{d} \kappa\right)=D^{\prime}\left(t_{1}\right) \cdot t_{1}<0 \quad \forall t_{1} \in[0, T], \\
\frac{\mathrm{d} Q_{1 \mid s_{1}}\left(t_{1}\right)}{\mathrm{d} t_{1}} & =\frac{\mathrm{d}}{\mathrm{d} t_{1}}\left(\int_{D(0)-D\left(t_{1}\right)}^{D(0)} R(\kappa) \mathrm{d} \kappa\right)=-R^{\prime}\left(D(0)-D\left(t_{1}\right)\right) \cdot D^{\prime}\left(t_{1}\right)<0 \quad \forall t_{1} \in[0, T],
\end{aligned}
$$

it becomes obvious that $l\left(t_{1}\right)$ is monotone decreasing on its domain, i.e. $\frac{\mathrm{d} l\left(t_{1}\right)}{\mathrm{d} t_{1}}<0 \forall t_{1} \in[0 ; T]$. Function $r\left(t_{1}\right)$ can be considered as being of the form $r\left(t_{1}\right)=f\left(t_{1}\right) / t_{1}$, where $f\left(t_{1}\right)$ is defined as the function $f\left(t_{1}\right):=r\left(t_{1}\right) \cdot t_{1}$. We obtain for $f(0)$ and the boundaries of $r\left(t_{1}\right)$

$$
f(0)=\mathbf{P}\left(s_{1}\right) T Q_{E}\left(\mathbf{E}\left[\tilde{z} \mid s_{1}\right] \mathbf{P}\left(s_{0}\right)\left(\bar{c}_{1 \mid s_{1}}-\bar{c}_{1 \mid s_{0}}\right)-\sigma_{1 \mid s_{1}}^{2}+\sigma_{12 \mid s_{1}}\right)
$$

\footnotetext{
${ }^{14}$ Note that the derivative $\frac{\mathrm{d} \mathcal{L}_{v}\left(t_{1}, t_{2}\left(t_{1}\right)\right)}{\mathrm{d} t_{1}}$ equals the directional derivative $\nabla_{\vec{t}} \mathcal{L}_{v}\left(K_{1}, K_{2}\right)=\frac{\partial \mathcal{L}_{v}\left(K_{1}, K_{2}\right)}{\partial K_{1}} \cdot t_{1}+\frac{\partial \mathcal{L}_{v}\left(K_{1}, K_{2}\right)}{\partial K_{2}} \cdot t_{2}\left(t_{1}\right)$ with the directional vector $\vec{t}=\left(t_{1}, t_{2}\left(t_{1}\right)\right)^{T}$ of unit length, i.e. $|\vec{t}|=1$.
} 


$$
r(T)=0, \quad \lim _{t_{1} \searrow 0} r\left(t_{1}\right)=\left\{\begin{aligned}
+\infty & \text { for } f(0)>0 \\
0 & \text { for } f(0)=0 \\
-\infty & \text { for } f(0)<0
\end{aligned}\right.
$$

Furthermore, it can be concluded that $r\left(t_{1}\right)$ is monotone increasing (and decreasing, respectively) in an interval $(0 ; \xi)$ for arbitrary means and variances, before it may start to decrease if $f(0)<0$ (and increase if $f(0)>0$, respectively). Thereby, non-monotony of $r\left(t_{1}\right)$ can only occur if

$$
g:=\sigma_{2 \mid s_{1}}^{2}-\sigma_{12 \mid s_{1}}+\mathbf{P}\left(s_{0}\right)\left(\bar{c}_{2 \mid s_{1}}-\bar{c}_{2 \mid s_{0}}\right) \mathbf{E}\left[\tilde{z} \mid s_{1}\right]\left\{\begin{array}{l}
>0 \text { for } f(0)<0 \\
<0 \text { for } f(0)>0
\end{array}\right.
$$

where $g$ refers to the last summand of $f\left(t_{1}\right)$.

Since an explicit formulation of the stationary points is not possible and the problem is non-convex in variable $t_{1}$, we use the first derivatives to test whether one of the possibly multiple stationary points is a local minimum or a local maximum: If there exists a $r \in \mathbb{R}_{+}$such that for every $t_{1} \in\left(t_{1}^{o}-r, t_{1}^{o}\right]$ it holds $\frac{\partial \mathcal{L}_{v}}{\partial K_{2}}\left(t_{1}\right)-\frac{\partial \mathcal{L}_{v}}{\partial K_{1}}\left(t_{1}\right) \leq 0$, and for every $t_{1} \in\left[t_{1}^{o}, t_{1}^{o}+r\right)$ it is $\frac{\partial \mathcal{L}_{v}}{\partial K_{2}}\left(t_{1}\right)-\frac{\partial \mathcal{L}_{v}}{\partial K_{1}}\left(t_{1}\right) \geq 0$, then $\mathcal{L}_{v}$ has a local minimum at $t_{1}^{o}$ according to the mean value theorem. Consequently, a local minimum in $t_{1}^{o}$ implies that $r\left(t_{1}\right)-l\left(t_{1}\right) \leq 0, \forall t_{1} \in\left(t_{1}^{o}-r, t_{1}^{o}\right]$ and $r\left(t_{1}\right)-l\left(t_{1}\right) \geq 0, \forall t_{1} \in\left[t_{1}^{o}, t_{1}^{o}+r\right)$. The obtained stationary points $t_{1}^{o} \in(0 ; T)$ with $l\left(t_{1}^{o}\right)=r\left(t_{1}^{o}\right)$ and therewith $\frac{\partial \mathcal{L}_{v}}{\partial K_{1}}\left(t_{1}^{o}\right)=\frac{\partial \mathcal{L}_{v}}{\partial K_{2}}\left(t_{1}^{o}\right)=0$ can be characterized as follows corresponding to Figure B.16:

I) For $l(0) \geq 0 \wedge f(0) \leq 0$, there may exist up to two stationary points $t_{1}^{o}$ :

- For two stationary points to exist, it is required $g>0$. Then $\mathcal{L}_{v}\left(t_{1}^{o}\right)$ takes a minimum if $r\left(t_{1}\right)-$ $l\left(t_{1}\right) \leq 0, \forall t_{1} \in\left(t_{1}^{o}-r, t_{1}^{o}\right]$ and $r\left(t_{1}\right)-l\left(t_{1}\right) \geq 0, \forall t_{1} \in\left[t_{1}^{o}, t_{1}^{o}+r\right)$. Hence, the efficient portfolio consists of both technologies.

- For $g \leq 0 \wedge l(T) \leq 0$ there exists a unique local minimum at $t_{1}^{o}$ and the variance efficient portfolio consists of a combination of both technologies.

- Otherwise, if a feasible stationary point $t_{1}^{o}$ does not exist, the variance efficient portfolio consists only of technology $2 .{ }^{15}$

II) For $l(0)<0 \wedge f(0) \leq 0$ (equivalent to condition (42)), there exists a unique local minimum at $t_{1}^{o}$. Hence, the variance efficient portfolio consists of both technologies.

\footnotetext{
${ }^{15}$ Since $l(T) \geq 0$ requires the variance of technology 1 to be much greater than the variance of technology 2 , it can be concluded that the variance efficient portfolio will only consist of technology 2 , not of technology 1 .
} 
For $f(0)<0$

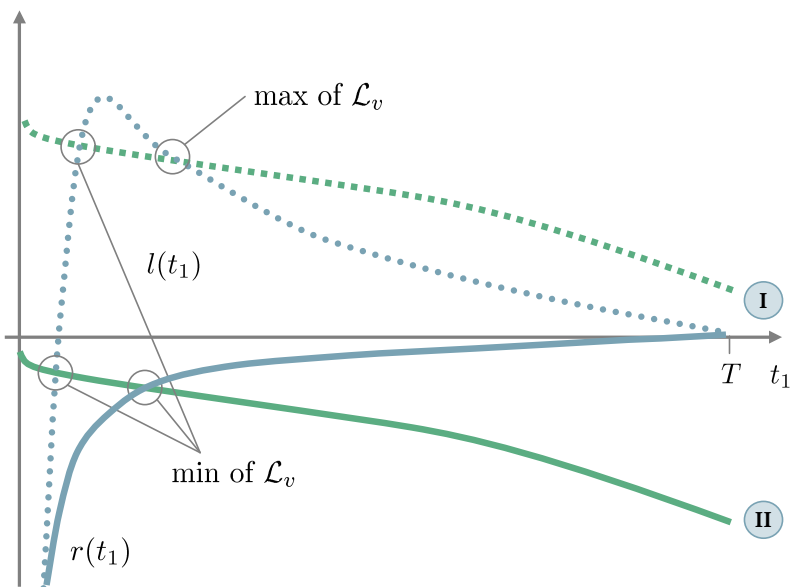

For $f(0)>0$

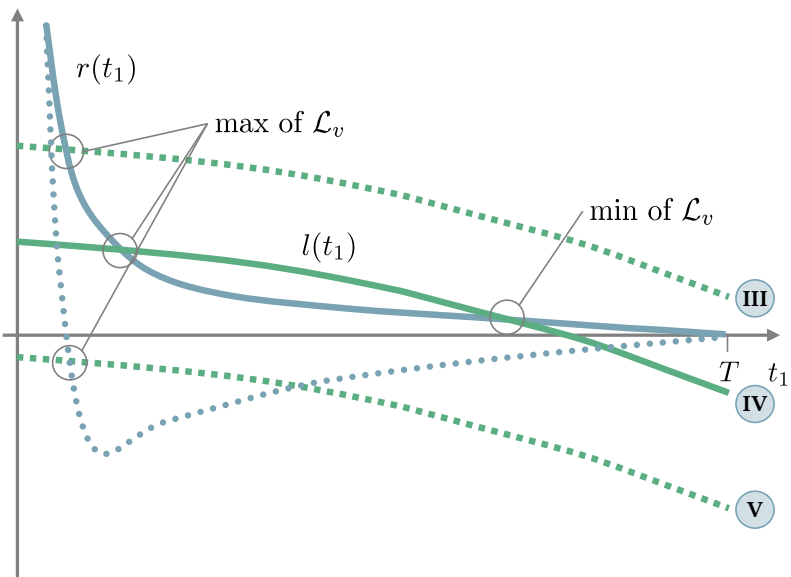

Figure B.16: Stationarity conditions for the purely variance-minimal portfolio in the case $\lambda>0$. The intersection of $l\left(t_{1}\right)$ (green line) and $r\left(t_{1}\right)$ (blue line) represents the optimal operating time.

III) For $l(T) \geq 0 \wedge f(0)>0$, there exists a unique local maximum at $t_{1}^{o}$, since it is $r\left(t_{1}\right)-l\left(t_{1}\right) \geq 0, \forall t_{1} \in$ $\left(t_{1}^{o}-r, t_{1}^{o}\right]$ and $r\left(t_{1}\right)-l\left(t_{1}\right) \leq 0, \forall t_{1} \in\left[t_{1}^{o}, t_{1}^{o}+r\right)$. Hence, it can be concluded that the variance efficient portfolio consists only of technology 2 .

IV) For $l(T)<0 \wedge l(0)>0 \wedge(f(0)>0)$, there may exist up two feasible stationary points $t_{1}^{o}$ :

- In case of two stationary points, $\mathcal{L}_{v}\left(t_{1}^{o}\right)$ takes the minimum if $r\left(t_{1}\right)-l\left(t_{1}\right) \leq 0, \forall t_{1} \in\left(t_{1}^{o}-r, t_{1}^{o}\right]$ and $r\left(t_{1}\right)-l\left(t_{1}\right) \geq 0, \forall t_{1} \in\left[t_{1}^{o}, t_{1}^{o}+r\right)$. Hence, the efficient portfolio consists of both technologies.

- Otherwise, if a feasible stationary point $t_{1}^{o}$ does not exist, the variance efficient portfolio consists only of technology 1 .

V) For $l(0) \leq 0 \wedge f(0)>0$, there may exist up two feasible stationary points $t_{1}^{o}$ :

- For two stationary points to exist, it is required $g<0$. Then, $\mathcal{L}_{v}\left(t_{1}^{o}\right)$ takes the minimum if $r\left(t_{1}\right)-l\left(t_{1}\right) \leq 0, \forall t_{1} \in\left(t_{1}^{o}-r, t_{1}^{o}\right]$ and $r\left(t_{1}\right)-l\left(t_{1}\right) \geq 0, \forall t_{1} \in\left[t_{1}^{o}, t_{1}^{o}+r\right)$. In this case, the efficient portfolio consists of both technologies.

- Otherwise, if a feasible stationary point $t_{1}^{o}$ does not exist (for which $g>0$ is sufficient), the variance efficient portfolio consists only of technology $1 .^{16}$

\footnotetext{
${ }^{16}$ Since this case requires the variance of technology 2 to be much greater than the variance of technology 1 , the efficient portfolio consists of technology 1 and not of technology 2 .
} 
Appendix B.5. Standard solutions to the combined portfolio problem with uncertainty in the merit order Proof of Eqs. (44) and (45). For the case with two technologies, the KKT conditions (44) and (45) can be derived from the Lagrangian (43) as follows:

$$
\begin{aligned}
\frac{\partial \mathcal{L}}{\partial K_{1}}= & c_{i n v, 1}-\lambda-\mathbf{P}\left(s_{0}\right) t_{1}\left(\sum _ { u = 1 } ^ { 2 } ( - 1 ) ^ { u } \left(\mathbf{E}\left[\tilde{c}_{u} \mid s_{0}\right]+A Q_{u \mid s_{0}}\left(\operatorname{Var}\left[\tilde{c}_{u} \mid s_{0}\right]-\operatorname{Cov}\left[\tilde{c}_{1}, \tilde{c}_{2} \mid s_{0}\right]\right.\right.\right. \\
& \left.\left.\left.+\mathbf{P}\left(s_{1}\right)\left(\mathbf{E}\left[\tilde{c}_{u} \mid s_{0}\right]^{2}-\mathbf{E}\left[\tilde{c}_{2} \mid s_{0}\right] \mathbf{E}\left[\tilde{c}_{1} \mid s_{0}\right]-\mathbf{E}\left[\tilde{c}_{u} \mid s_{0}\right] \sum_{v=1}^{2} \mathbf{E}\left[\tilde{c}_{v} \mid s_{1}\right] \frac{Q_{v \mid s_{1}}}{Q_{u \mid s_{0}}}\right)\right)\right)\right) \\
= & t_{1} \mathbf{P}\left(s_{0}\right) A\left(Q_{1 \mid s_{0}}\left(\operatorname{Var}\left[\tilde{z} \mid s_{0}\right]+\mathbf{P}\left(s_{1}\right) \mathbf{E}\left[\tilde{z} \mid s_{0}\right]^{2}\right)\right. \\
& \left.-Q_{E}\left(\sigma_{2 \mid s_{0}}^{2}-\sigma_{12 \mid s_{0}}+\mathbf{P}\left(s_{1}\right)\left(\bar{c}_{2 \mid s_{0}}-\bar{c}_{2 \mid s_{1}}\right) \mathbf{E}\left[\tilde{z} \mid s_{0}\right]\right)\right) \\
& -t_{1} \mathbf{P}\left(s_{0}\right) \mathbf{E}\left[\tilde{z} \mid s_{0}\right]\left(\mathbf{P}\left(s_{1}\right) Q_{1 \mid s_{1}} \mathbf{E}\left[\tilde{z} \mid s_{1}\right]+1\right)+c_{i n v, 1}-\lambda \\
\frac{\partial \mathcal{L}}{\partial K_{2}}= & c_{i n v, 2}-\lambda+\mathbf{P}\left(s_{1}\right) t_{2}\left(\sum _ { u = 1 } ^ { 2 } ( - 1 ) ^ { u } \left(\mathbf{E}\left[\tilde{c}_{u} \mid s_{1}\right]+A Q_{u \mid s_{1}}\left(\operatorname{Var}\left[\tilde{c}_{u} \mid s_{1}\right]-\operatorname{Cov}\left[\tilde{c}_{1}, \tilde{c}_{2} \mid s_{1}\right]\right.\right.\right. \\
& \left.\left.\left.\left.+\mathbf{E}\left[\tilde{c}_{u} \mid s_{1}\right] \sum_{v=1}^{2} \mathbf{E}\left[\tilde{c}_{v} \mid s_{0}\right] \frac{Q_{v \mid s_{0}}}{Q_{u \mid s_{1}}}\right)\right)\right)\right) \\
= & t_{2} \mathbf{P}\left(s_{1}\right) \mathbf{E}\left[\tilde{z} \mid s_{1}\right]\left(A \mathbf{P}\left(s_{0}\right) Q_{1 \mid s_{0}} \mathbf{E}\left[\tilde{z} \mid s_{0}\right]+1\right)-t_{2} \mathbf{P}\left(s_{1}\right) A\left(Q_{1 \mid s_{1}}\left(\operatorname{Var}\left[\tilde{z} \mid s_{1}\right]+\mathbf{P}\left(s_{0}\right) \mathbf{E}\left[\tilde{z} \mid s_{1}\right]^{2}\right)\right. \\
& \left.-Q_{E}\left(\sigma_{2 \mid s_{1}}^{2}-\sigma_{12 \mid s_{1}}+\mathbf{P}\left(s_{0}\right)\left(\bar{c}_{2 \mid s_{1}}-\bar{c}_{2 \mid s_{0}}\right) \mathbf{E}\left[\tilde{z} \mid s_{1}\right]\right)\right)+c_{i n v, 2}-\lambda
\end{aligned}
$$

Proposition 2.3 (Existence and uniqueness of an interior solution for the case $\lambda \neq 0$ ). Let be $c_{\text {inv }, 1}>c_{\text {inv }, 2}$ and total installed capacity matching maximum demand, i.e. $\lambda \neq 0$ in constraint (12). If both technologies 1 and 2 are included in the purely cost-efficient portfolio (i.e. $A=0$ ) and in the purely variance-efficient portfolio (i.e. $A \rightarrow+\infty$ ) satisfying condition (42), then all cost-variance efficient portfolios with $A>0$ corresponding to problem (9)-(12) consist of a unique combination of both technologies.

Proof of Proposition 2.3. According to the assumption $\lambda \neq 0, t_{2}$ can be expressed as a function of the $t_{1}$ throughout this proof with $t_{2}\left(t_{1}\right)=R\left(D(0)-D\left(t_{1}\right)\right)$. Herewith, the solution condition $v\left(t_{1}\right)=0$ in Eqn. (47) can be derived as follows: For an interior solution with $K_{1}^{*}, K_{2}^{*}>0$, KKT conditions (44) and (45) have to be satisfied with equality as necessary optimality condition. Eliminating $\lambda$ through subtraction of these conditions yields $\frac{\partial \mathcal{L}}{\partial K_{1}}-\frac{\partial \mathcal{L}}{\partial K_{2}}=0$. For $t_{1}>0$, the latter condition may equivalently be written as 
$l_{3}\left(t_{1}\right)=r_{3}\left(t_{1}\right)$ defined by

$$
\begin{aligned}
l_{3}\left(t_{1}\right):= & \mathbf{P}\left(s_{0}\right) A\left(Q_{1 \mid s_{0}}\left(\operatorname{Var}\left[\tilde{z} \mid s_{0}\right]+\mathbf{P}\left(s_{1}\right) \mathbf{E}\left[\tilde{z} \mid s_{0}\right]^{2}\right)-\left(\mathbf{P}\left(s_{1}\right) Q_{1 \mid s_{1}} \mathbf{E}\left[\tilde{z} \mid s_{1}\right]+1 / A\right) \mathbf{E}\left[\tilde{z} \mid s_{0}\right]\right) \\
& +\frac{c_{i n v, 1}-c_{i n v, 2}}{t_{1}} \\
r_{3}\left(t_{1}\right):= & \mathbf{P}\left(s_{0}\right) A Q_{E}\left(\sigma_{2 \mid s_{0}}^{2}-\sigma_{12 \mid s_{0}}+\mathbf{P}\left(s_{1}\right)\left(\bar{c}_{2 \mid s_{0}}-\bar{c}_{2 \mid s_{1}}\right) \mathbf{E}\left[\tilde{z} \mid s_{0}\right]\right) \\
& +\mathbf{P}\left(s_{1}\right) A_{\frac{t_{2}}{t_{1}}}\left(Q_{E}\left(\sigma_{2 \mid s_{1}}^{2}-\sigma_{12 \mid s_{1}}+\mathbf{P}\left(s_{0}\right)\left(\bar{c}_{2 \mid s_{1}}-\bar{c}_{2 \mid s_{0}}\right) \mathbf{E}\left[\tilde{z} \mid s_{1}\right]\right)\right. \\
& \left.-\left(\operatorname{Var}\left[\tilde{z} \mid s_{1}\right]+\mathbf{P}\left(s_{0}\right) \mathbf{E}\left[\tilde{z} \mid s_{1}\right]^{2}\right) Q_{1 \mid s_{1}}+\left(\mathbf{P}\left(s_{0}\right) \mathbf{E}\left[\tilde{z} \mid s_{0}\right] Q_{1 \mid s_{0}}+1 / A\right) \mathbf{E}\left[\tilde{z} \mid s_{1}\right]\right)
\end{aligned}
$$

By taking into account the sign of the derivatives as shown in Eqn. (B.34)-(B.36) in the previous proof, the behavior of $l_{3}\left(t_{1}\right), r_{3}\left(t_{1}\right)$ can be characterized at the boundaries as follows:

$$
\begin{aligned}
& \lim _{t_{1} \searrow 0} l_{3}\left(t_{1}\right)=\left\{\begin{aligned}
+\infty & \text { for } c_{i n v, 1}>c_{i n v, 2} \\
0 & \text { for } c_{i n v, 1}=c_{i n v, 2}, \\
-\infty & \text { for } c_{i n v, 1}<c_{i n v, 2}
\end{aligned}\right. \\
& l_{3}(T)= \frac{c_{i n v, 1}-c_{i n v, 2}}{T}-\mathbf{P}\left(s_{0}\right) \mathbf{E}\left[\tilde{z} \mid s_{0}\right], \\
& \lim _{t_{1} \searrow 0} r_{3}\left(t_{1}\right)=\left\{\begin{aligned}
+\infty & \text { for } f_{1}(0)>0 \\
0 & \text { for } f_{1}(0)=0, \\
-\infty & \text { for } f_{1}(0)<0
\end{aligned}\right. \\
& r_{3}(T)=\mathbf{P}\left(s_{0}\right) A Q_{E}\left(\sigma_{2 \mid s_{0}}^{2}-\sigma_{12 \mid s_{0}}+\mathbf{P}\left(s_{1}\right)\left(\bar{c}_{2 \mid s_{0}}-\bar{c}_{2 \mid s_{1}}\right) \mathbf{E}\left[\tilde{z} \mid s_{0}\right]\right)
\end{aligned}
$$

with the definition $f_{1}(0):=\mathbf{E}\left[\tilde{z} \mid s_{1}\right] \mathbf{P}\left(s_{0}\right)\left(\bar{c}_{1 \mid s_{1}}-\bar{c}_{1 \mid s_{0}}\right)+\frac{\mathbf{E}\left[\tilde{z} \mid s_{1}\right]}{Q_{E} A}-\sigma_{1 \mid s_{1}}^{2}+\sigma_{12 \mid s_{1}}$.

In case $c_{i n v, 1}>c_{i n v, 2}$, it can be concluded that $\frac{\mathrm{d} l_{3}\left(t_{1}\right)}{\mathrm{d} t_{1}}<0$ and hence $l_{3}\left(t_{1}\right)$ is monotone decreasing as shown in Figure B.17. From our initial assumption that the purely cost-minimal portfolio consists of both technologies, we can further conclude $l_{3}(T)<0$ according to Proposition 2.1. Thus, a unique intersection point of $l_{3}\left(t_{1}\right)$ and $r_{3}\left(t_{1}\right)$ and therewith a unique interior solution with $0<t_{1}^{*}, t_{2}^{*}<T$ is obtained if and only if $r_{3}(T)>l_{3}(T)$. Given that the variance-minimal portfolio consists of both technologies satisfying condition (42), it can be concluded $f(0) \leq 0$. This implies also $f_{1}(0) \leq$ and therewith $\lim _{t_{1} \searrow_{0}} r_{3}\left(t_{1}\right) \leq 0$. Furthermore, it follows $r_{3}(T)>0$ because the purely variance efficient portfolio consists of technology 2 if and only if $r_{3}(T) \leq 0$ according to Proposition 2.2 .

Assuming $c_{i n v, 1}<c_{i n v, 2}$ yields again $l_{3}(T)<0$ and with the purely variance-minimal portfolio consisting of both technologies $r_{3}(T)>0$. Hence a unique intersection point of $l_{3}\left(t_{1}\right)$ and $r_{3}\left(t_{1}\right)$ (cf. Figure B.17) and 
$c_{i n v, 1}>c_{i n v, 2}$

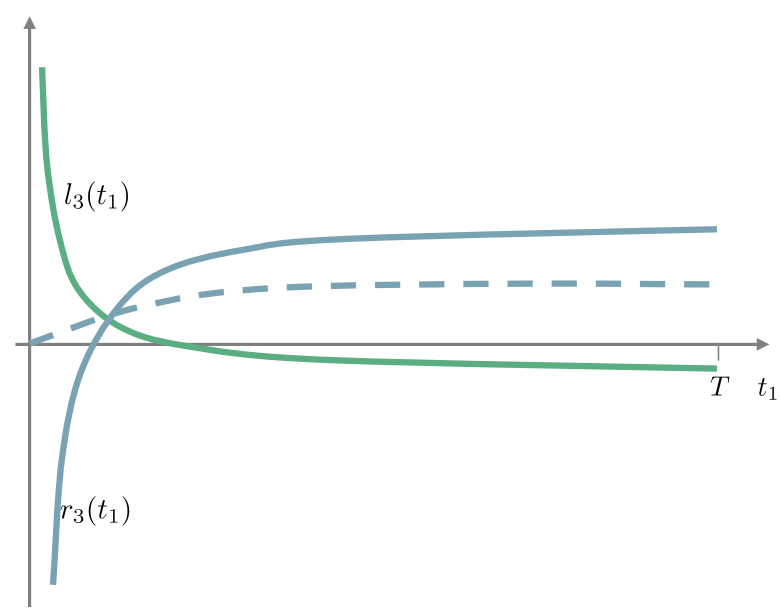

$c_{i n v, 1}<c_{i n v, 2}$

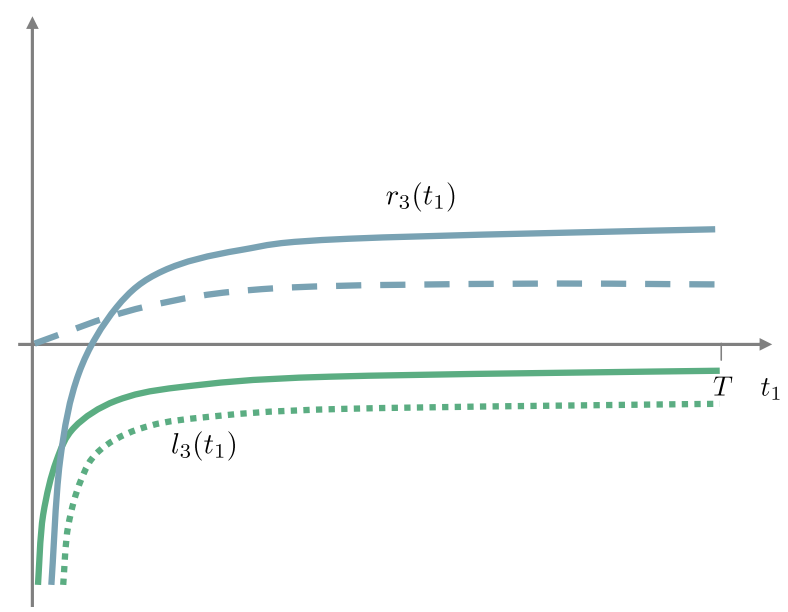

Figure B.17: Stationarity conditions for the cost-variance efficient portfolio in the cases $c_{i n v, 1}>c_{i n v, 2}$ (left) and $c_{i n v, 1}<c_{i n v, 2}$ (right). The intersection of $l_{3}\left(t_{1}\right)$ (green line) and $r_{3}\left(t_{1}\right)$ (blue line) represents the optimal operating time.

therewith a unique interior solution with $0<t_{1}^{*}, t_{2}^{*}<T$ is obtained if and only if

$$
\begin{aligned}
\lim _{t_{1} \searrow 0} r_{3}\left(t_{1}\right)-l_{3}\left(t_{1}\right) & \leq 0 \\
\Leftrightarrow \quad f_{1}(0)-c_{i n v, 1}+c_{i n v, 2} & \leq 0 \\
\Leftrightarrow \quad f(0)+\frac{\mathbf{E}\left[\tilde{z} \mid s_{1}\right]}{Q_{E} A}-c_{i n v, 1}+c_{i n v, 2} & \leq 0
\end{aligned}
$$

Notably, the latter inequality is however only satisfied if $A$ is greater than a defined threshold $A_{0}$. This is why for $c_{i n v, 1}>c_{i n v, 2}$, the cost-variance efficient portfolio does not necessarily consist of both technologies.

Proposition 2.4 (Implicit functions). For the implicit function $\frac{\partial \mathcal{L}}{\partial K_{1}}\left(\check{t}_{1}, \check{t}_{2}\right)=0$ with $\frac{\partial \mathcal{L}}{\partial K_{1}}\left(t_{1}, t_{2}\right):[0, T] \times$ $[0, T] \rightarrow Z_{1} \subseteq \mathbb{R}_{+}$, there exists a unique function $\zeta_{1}\left(t_{2}\right):(0, T] \rightarrow Z_{1} \subseteq \mathbb{R}_{+}$. Similarly, for the implicit function $\frac{\partial \mathcal{L}}{\partial K_{2}}\left(\check{t}_{1}, \check{t}_{2}\right)=0$ with $\frac{\partial \mathcal{L}}{\partial K_{2}}\left(t_{1}, t_{2}\right):[0, T] \times[0, T] \rightarrow Z_{2} \subseteq \mathbb{R}_{+}$, there exists a unique function $\zeta_{2}\left(t_{1}\right)$ : $(0, T] \rightarrow Z_{2} \subseteq \mathbb{R}_{+}$.

Proof of Proposition 2.4. According to our initial assumptions $D(t)$ is strictly monotone decreasing, the likelihood for a fuel switch is non-negative (i.e. $\mathbf{P}\left(s_{0}\right) \geq 0$ ) and all individuals act strictly risk averse (i.e. $A>0$ ). By partial differentiation of Eqs. (44), (45) we obtain

$$
\frac{\partial}{\partial t_{i}} \frac{\partial \mathcal{L}}{\partial K_{i}}\left(t_{1}, t_{2}\right)=A t_{1} t_{2} \mathbf{P}\left(s_{1}\right) \mathbf{P}\left(s_{0}\right) \cdot\left(c_{1 \mid s_{0}}-c_{2 \mid s_{0}}\right)\left(c_{1 \mid s_{1}}-c_{2 \mid s_{1}}\right) \frac{\mathrm{d} D\left(t_{i}\right)}{\mathrm{d} t_{i}} \neq 0 \quad \forall t_{i}>0,(i=1,2)
$$

According to the well-known implicit function theorem, the existence of the functions $t_{1}=\zeta_{1}\left(t_{2}\right)$ and $t_{2}=\zeta_{2}\left(t_{1}\right)$ is hence given for $t_{2}>0$ and $t_{1}>0$, respectively. 
Proposition 2.5 (Monotony). For given expected fuel prices $\bar{c}_{1 \mid s_{0}} \leq \bar{c}_{2 \mid s_{0}}$ and $\bar{c}_{2 \mid s_{1}} \leq \bar{c}_{1 \mid s_{1}}$ and $\zeta_{1}\left(t_{2}\right)$ and $\zeta_{2}\left(t_{1}\right)$ being functions represented by the implicit functions $\frac{\partial \mathcal{L}}{\partial K_{1}}\left(t_{1}, t_{2}\right)=0$ from Eqn. (44), and $\frac{\partial \mathcal{L}}{\partial K_{2}}\left(t_{1}, t_{2}\right)=$ 0 from Eqn. (45), respectively, $\zeta_{1}\left(t_{2}\right)$ and $\zeta_{2}\left(t_{1}\right)$ are both monotone increasing in $t_{2}$ and $t_{1}$, respectively. For $\bar{c}_{1 \mid s_{0}}<\bar{c}_{2 \mid s_{0}}$ and $\bar{c}_{2 \mid s_{1}}<\bar{c}_{1 \mid s_{1}}$, it follows strict monotony of $\zeta_{1}\left(t_{2}\right)$ and $\zeta_{2}\left(t_{1}\right)$.

Proof of Proposition 2.5. The necessary condition for an interior solution is given by the nonlinear equation system with Eqs. (44), $\frac{\partial \mathcal{L}}{\partial K_{1}}=0$, and (45), $\frac{\partial \mathcal{L}}{\partial K_{2}}=0$ with both equations representing functions of $K_{1}, K_{2}$, and $t_{1}, t_{2}$, respectively. By rewriting and applying $Q_{1 \mid s_{i}}=Q_{E}-Q_{2 \mid s_{i}},(i=0,1)$, we can bring optimality condition from Eqs. (44), (45) in a form where $t_{1}, t_{2}$ are separated to the two sides of the equation, i.e.

$$
\frac{\partial \mathcal{L}}{\partial K_{1}}\left(t_{1}, t_{2}\right)=0 \Leftrightarrow l_{1}\left(t_{1}\right)=r_{1}\left(t_{2}\right) \quad \text { and } \quad \frac{\partial \mathcal{L}}{\partial K_{2}}\left(t_{1}, t_{2}\right)=0 \Leftrightarrow l_{2}\left(t_{2}\right)=r_{2}\left(t_{1}\right)
$$

Then, we obtain

$$
\begin{aligned}
l_{1}\left(t_{1}\right):= & \frac{c_{i n v, 1}}{t_{1} \mathbf{P}\left(s_{0}\right)}+A Q_{1 \mid s_{0}}\left(\operatorname{Var}\left[\tilde{z} \mid s_{0}\right]+\mathbf{P}\left(s_{1}\right) \mathbf{E}\left[\tilde{z} \mid s_{0}\right]^{2}\right) \\
& -A Q_{E}\left(\sigma_{2 \mid s_{0}}^{2}-\sigma_{12 \mid s_{0}}+\mathbf{P}\left(s_{1}\right)\left(\bar{c}_{2 \mid s_{0}}-\bar{c}_{2 \mid s_{1}}\right) \mathbf{E}\left[\tilde{z} \mid s_{0}\right]\right) \\
r_{1}\left(t_{2}\right):= & \mathbf{E}\left[\tilde{z} \mid s_{0}\right]\left(A \mathbf{P}\left(s_{1}\right) Q_{1 \mid s_{1}} \mathbf{E}\left[\tilde{z} \mid s_{1}\right]+1\right) \\
l_{2}\left(t_{2}\right):= & \frac{c_{i n v, 2}}{t_{2} \mathbf{P}\left(s_{1}\right)}-A Q_{1 \mid s_{1}}\left(\operatorname{Var}\left[\tilde{z} \mid s_{1}\right]+\mathbf{P}\left(s_{0}\right) \mathbf{E}\left[\tilde{z} \mid s_{1}\right]^{2}\right) \\
& +A Q_{E}\left(\sigma_{2 \mid s_{1}}^{2}-\sigma_{12 \mid s_{1}}+\mathbf{P}\left(s_{0}\right)\left(\bar{c}_{2 \mid s_{1}}-\bar{c}_{2 \mid s_{0}}\right) \mathbf{E}\left[\tilde{z} \mid s_{1}\right]\right) \\
r_{2}\left(t_{1}\right):= & -\mathbf{E}\left[\tilde{z} \mid s_{1}\right]\left(A \mathbf{P}\left(s_{0}\right) Q_{1 \mid s_{0}} \mathbf{E}\left[\tilde{z} \mid s_{0}\right]+1\right)
\end{aligned}
$$

The first derivatives of $l_{1}\left(t_{1}\right), r_{1}\left(t_{2}\right), l_{2}\left(t_{2}\right), r_{2}\left(t_{1}\right)$ can be derived as follows:

$$
\begin{array}{rlrl}
\frac{\mathrm{d} l_{1}\left(t_{1}\right)}{\mathrm{d} t_{1}} & =\frac{-c_{i n v, 1}}{t_{1}^{2} \mathbf{P}\left(s_{0}\right)}+A t_{1} \cdot\left(\operatorname{Var}\left[\tilde{z} \mid s_{0}\right]+\mathbf{P}\left(s_{1}\right) \mathbf{E}\left[\tilde{z} \mid s_{0}\right]^{2}\right)\left(\frac{\mathrm{d} D\left(t_{1}\right)}{\mathrm{d} t_{1}}\right) \leq 0 & & \forall t_{1}>0 \\
\frac{\mathrm{d} r_{1}\left(t_{2}\right)}{\mathrm{d} t_{2}}=-A t_{2} \mathbf{P}\left(s_{1}\right) \cdot \mathbf{E}\left[\tilde{z} \mid s_{1}\right] \mathbf{E}\left[\tilde{z} \mid s_{0}\right]\left(\frac{\mathrm{d} D\left(t_{2}\right)}{\mathrm{d} t_{2}}\right) \leq 0 & & \forall t_{2}>0 \\
\frac{\mathrm{d} l_{2}\left(t_{2}\right)}{\mathrm{d} t_{2}}=\frac{-c_{i n v, 2}}{t_{2}^{2} \mathbf{P}\left(s_{1}\right)}+A t_{2} \cdot\left(\operatorname{Var}\left[\tilde{z} \mid s_{1}\right]+\mathbf{P}\left(s_{0}\right) \mathbf{E}\left[\tilde{z} \mid s_{1}\right]^{2}\right)\left(\frac{\mathrm{d} D\left(t_{2}\right)}{\mathrm{d} t_{2}}\right) \leq 0 & & \forall t_{2}>0 \\
\frac{\mathrm{d} r_{2}\left(t_{1}\right)}{\mathrm{d} t_{1}}=-A t_{1} \mathbf{P}\left(s_{0}\right) \cdot \mathbf{E}\left[\tilde{z} \mid s_{0}\right] \mathbf{E}\left[\tilde{z} \mid s_{1}\right]\left(\frac{\mathrm{d} D\left(t_{1}\right)}{\mathrm{d} t_{1}}\right) \leq 0 & & \forall t_{1}>0
\end{array}
$$

Since it holds for all feasible parameter sets $\frac{\mathrm{d} l_{1}}{\mathrm{~d} t_{1}} \leq 0$ and $\frac{\mathrm{d} r_{1}}{\mathrm{~d} t_{2}} \leq 0$, it follows for the equation $l_{1}\left(t_{1}\right)-r_{1}\left(t_{2}\right)=0$ that $t_{1}$ must (strictly) monotone increase (decrease) for (strictly) monotone increasing (decreasing) variable $t_{2}$. Consequently, the corresponding (explicit) function $\zeta_{1}\left(t_{2}\right)$ is (strictly) monotone increasing in $t_{2}$. In the same way, it can be concluded from $\frac{\mathrm{d} l_{2}}{\mathrm{~d} t_{1}} \leq 0$ and $\frac{\mathrm{d} r_{2}}{\mathrm{~d} t_{2}} \leq 0$ that $\zeta_{2}\left(t_{1}\right)$ is strictly monotone increasing in $t_{1}$.

Proposition 2.6 (Existence and uniqueness of an interior solution for the case $\lambda=0$ ). If the technology 
parameters satisfy

$$
\begin{aligned}
& \frac{c_{i n v, 1}}{T \mathbf{P}\left(s_{0}\right)}-\mathbf{E}\left[\tilde{z} \mid s_{0}\right]-A Q_{E}\left(\sigma_{2 \mid s_{0}}^{2}-\sigma_{12 \mid s_{0}}+\mathbf{P}\left(s_{1}\right) \mathbf{E}\left[\tilde{z} \mid s_{0}\right]\left(\bar{c}_{2 \mid s_{0}}-\bar{c}_{1 \mid s_{1}}\right)\right)<0 \\
\text { and } \quad & \frac{c_{i n v, 2}}{T \mathbf{P}\left(s_{1}\right)}+\mathbf{E}\left[\tilde{z} \mid s_{1}\right]-A Q_{E}\left(\sigma_{1 \mid s_{1}}^{2}-\sigma_{12 \mid s_{1}}+\mathbf{P}\left(s_{0}\right) \mathbf{E}\left[\tilde{z} \mid s_{1}\right]\left(\bar{c}_{2 \mid s_{0}}-\bar{c}_{1 \mid s_{1}}\right)\right)<0
\end{aligned}
$$

then the cost-variance efficient portfolios with $A>0$ corresponding to problem (9)-(12) consist of a unique combination of both technologies. The total installed generation capacity of the cost-risk efficient portfolio may exceed total demand, implying $\lambda=0$ in constraint (12).

Proof of Proposition 2.6. Necessary prerequisite for an interior solution is the existence of at least one stationary tuple $\left(t_{1}^{o}, t_{2}^{o}\right) \in(0 ; T) \times(0 ; T)$ with $\zeta_{1}\left(t_{2}^{o}\right)=t_{1}^{o}$ and $\zeta_{2}\left(t_{1}^{o}\right)=t_{2}^{o}$. This tuple represents graphically the intersection point of $\zeta_{2}\left(t_{2}\right)$ and $\zeta_{1}\left(t_{2}\right)$. The existence of a unique stationary point $\left(t_{1}^{o}, t_{2}^{o}\right)$ can be shown in two steps: (a) At first, it can be proved that it holds $\zeta_{1}\left(t_{2}=0\right)>0$ and $\zeta_{2}\left(t_{1}=0\right)>0$. (b) Secondly, it can be shown that it is $\zeta_{1}\left(t_{2}=T\right)<T$ and $\zeta_{2}\left(t_{1}=T\right)<T$ for a defined set of parameters. Taking into account the monotony of $\zeta_{1}\left(t_{2}\right), \zeta_{2}\left(t_{1}\right)$ (cf. Proposition 2.5), this consequently implies the existence of a unique intersection point. Therefore, we next consider the limits of the local functions $\zeta_{1}\left(t_{2}\right), \zeta_{2}\left(t_{1}\right)$ :

a) First, $\zeta_{2}\left(t_{1}=0\right)>0$ can be concluded from

$$
\lim _{t_{2} \rightarrow 0} l_{2}\left(t_{2}\right)=\infty \text { and } \lim _{t_{1} \rightarrow \xi} r_{2}\left(t_{1}\right)<\infty \forall \xi \in[0, T]
$$

Similarly, $\zeta_{1}\left(t_{2}=0\right)>0$ can be concluded since it holds

$$
\lim _{t_{1} \rightarrow 0} l_{1}\left(t_{1}\right)=\infty \text { and } \lim _{t_{2} \rightarrow \xi} r_{1}\left(t_{2}\right)<\infty \forall \xi \in[0, T] .
$$

b) Next, we will derive a condition which is sufficient for $\zeta_{1}\left(t_{2}=T\right)<T$ and $\zeta_{2}\left(t_{1}=T\right)<T$ : Note that $t_{1}=T$ implies $Q_{1 \mid s_{0}}=0, Q_{2 \mid s_{0}}=Q_{E}$, and similar $t_{2}=T$ implies $Q_{2 \mid s_{1}}=0, Q_{1 \mid s_{1}}=Q_{E}$. Since $l_{1}\left(t_{1}\right)$ is monotone decreasing, it can then be concluded that $\zeta_{1}\left(t_{2}=T\right)<T$ if and only if

$$
\begin{aligned}
& l_{1}\left(t_{1}=T\right)-r_{1}\left(t_{2}=T\right)<0 \\
& \Leftrightarrow \quad \frac{c_{i n v, 1}}{T \mathbf{P}\left(s_{0}\right)}-\mathbf{E}\left[\tilde{z} \mid s_{0}\right]-A Q_{E}\left(\sigma_{2 \mid s_{0}}^{2}-\sigma_{12 \mid s_{0}}+\mathbf{P}\left(s_{1}\right) \mathbf{E}\left[\tilde{z} \mid s_{0}\right]\left(\bar{c}_{2 \mid s_{0}}-\bar{c}_{1 \mid s_{1}}\right)\right)<0 .
\end{aligned}
$$

Similarly, it follows due to the monotony of $r_{2}\left(t_{1}\right)$ that $\zeta_{2}\left(t_{1}=T\right)<T$ if and only if

$$
\begin{aligned}
& l_{2}\left(t_{2}=T\right)-r_{2}\left(t_{1}=T\right)<0 \\
& \Leftrightarrow \quad \frac{c_{i n v, 2}}{T \mathbf{P}\left(s_{1}\right)}+\mathbf{E}\left[\tilde{z} \mid s_{1}\right]-A Q_{E}\left(\sigma_{1 \mid s_{1}}^{2}-\sigma_{12 \mid s_{1}}+\mathbf{P}\left(s_{0}\right) \mathbf{E}\left[\tilde{z} \mid s_{1}\right]\left(\bar{c}_{2 \mid s_{0}}-\bar{c}_{1 \mid s_{1}}\right)\right)<0 .
\end{aligned}
$$

Therewith, it must exist a stationary point $\left(t_{1}^{o}, t_{2}^{o}\right) \in(0 ; T) \times(0 ; T)$ with $\zeta_{1}\left(t_{2}^{o}\right)=t_{1}^{o}$ and $\zeta_{2}\left(t_{1}^{o}\right)=t_{2}^{o}$. Finally, it can be verified that the identified stationary point $\left(t_{1}^{o}, t_{2}^{o}\right) \in(0 ; T) \times(0 ; T)$ satisfying (B.55) and (B.56) 
represents a local minimum of the optimization problem. Utilizing the mean value theorem, $\mathcal{L}_{v}$ has a local minimum at $t_{1}^{o}$ it there exists a $r \in \mathbb{R}_{+}$such that for every tuple $\left(t_{1}^{o}, t_{2}^{o}\right) \in\left(t_{1}^{o}-r, t_{1}^{o}\right] \times\left(t_{2}^{o}-r, t_{2}^{o}\right]$ it is $\frac{\partial \mathcal{L}}{\partial K_{1}} \leq 0, \frac{\partial \mathcal{L}}{\partial K_{2}} \leq 0$, and for every $\left(t_{1}^{o}, t_{2}^{o}\right) \in\left[t_{1}^{o}, t_{1}^{o}+r\right) \times\left[t_{2}^{o}, t_{2}^{o}+r\right)$ it is $\frac{\partial \mathcal{L}}{\partial K_{1}} \geq 0, \frac{\partial \mathcal{L}}{\partial K_{2}} \geq 0$. By inserting, it can be verified

$$
\begin{aligned}
& \frac{\partial \mathcal{L}}{\partial K_{1}}\left(D(0), K_{2}\right)=c_{i n v, 1}>0 \\
& \frac{\partial \mathcal{L}}{\partial K_{1}}\left(0, K_{2}\right)=c_{i n v, 1}-\mathbf{P}\left(s_{0}\right) T \mathbf{E}\left[\tilde{z} \mid s_{0}\right]- \\
& -\mathbf{P}\left(s_{0}\right) T A\left(\mathbf{E}\left[\tilde{z} \mid s_{0}\right] \mathbf{P}\left(s_{1}\right)\left(Q_{1 \mid s_{1}} \mathbf{E}\left[\tilde{z} \mid s_{1}\right]+Q_{E}\left(\bar{c}_{2 \mid s_{0}}-\bar{c}_{2 \mid s_{1}}\right)\right)+Q_{E}\left(\sigma_{2 \mid s_{0}}^{2}-\sigma_{12 \mid s_{0}}\right)\right) \\
& <c_{i n v, 1}-\mathbf{P}\left(s_{0}\right) T\left(\mathbf{E}\left[\tilde{z} \mid s_{0}\right]+A Q_{E}\left(\sigma_{2 \mid s_{0}}^{2}-\sigma_{12 \mid s_{0}}+\mathbf{E}\left[\tilde{z} \mid s_{0}\right] \mathbf{P}\left(s_{1}\right)\left(\bar{c}_{2 \mid s_{0}}-\bar{c}_{1 \mid s_{1}}\right)\right)\right)<0 \\
& \frac{\partial \mathcal{L}}{\partial K_{2}}\left(K_{1}, D(0)\right)=c_{i n v, 2}>0 \\
& \frac{\partial \mathcal{L}}{\partial K_{2}}\left(K_{1}, 0\right)=c_{i n v, 2}+\mathbf{P}\left(s_{1}\right) T \mathbf{E}\left[\tilde{z} \mid s_{1}\right]- \\
& -\mathbf{P}\left(s_{1}\right) T A\left(\mathbf{E}\left[\tilde{z} \mid s_{1}\right] \mathbf{P}\left(s_{0}\right)\left(Q_{E}\left(\bar{c}_{2 \mid s_{0}}-\bar{c}_{1 \mid s_{1}}\right)-Q_{1 \mid s_{0}} \mathbf{E}\left[\tilde{z} \mid s_{0}\right]\right)+Q_{E}\left(\sigma_{1 \mid s_{1}}^{2}-\sigma_{12 \mid s_{1}}\right)\right) \\
& <c_{i n v, 2}+\mathbf{P}\left(s_{1}\right) T\left(\mathbf{E}\left[\tilde{z} \mid s_{1}\right]-A Q_{E}\left(\sigma_{1 \mid s_{1}}^{2}-\sigma_{12 \mid s_{1}}+\mathbf{E}\left[\tilde{z} \mid s_{1}\right] \mathbf{P}\left(s_{0}\right)\left(\bar{c}_{2 \mid s_{0}}-\bar{c}_{1 \mid s_{1}}\right)\right)\right)<0
\end{aligned}
$$

Consequently, condition (B.55)-(B.56) is sufficient for an interior local optimum with $\lambda=0$. Due to the convexity of the Lagrangian $\mathcal{L}$ in $\lambda$, the obtained local minimum is also a global minimum. 


\section{References}

Alexander, C., 1999. Correlation and cointegration in energy markets. In: Managing energy price risk. Risk publications.

BAFA, 2010. Bafa energy statistics. Bundesamt für Wirtschaft und Ausfuhrkontrolle (German Federal Office of Economics and Export Control).

URL http://www.bafa.de/

Bonilla, R., 2010. Cost-effective solutions to the world-wide acetonitrile shortage. PharmPro.

URL http://www.pharmpro.com/articles/2010/04/Cost-Effective-Solutions-to-the-World-Wide-AcetonitrileShortage/

Crew, M., Fernando, C., Kleindorfer, P., 1995. The theory of peak-load pricing: A survey. Journal of Regulatory Economics $8(3), 215-248$.

EEX, 2011. EEX historical data. European Energy Exchange.

URL http: //www.eex.com/

EIA, 2009. Petroleum data. Energy Information Administration.

URL http://www.eia.doe.gov/oil_gas/petroleum/info_glance/petroleum.html

Elishakoff, I., 1999. Probabilistic Theory of Structures. Dover Publications.

ENTSO-E, 2009. Consumption data, hourly load values Germany. European Network of Transmission System Operators for Electricity.

URL http://www . entsoe.eu/index.php?id=92

Eurostat, 2009. Energy statistics - prices.

URL http://epp.eurostat.ec.europa.eu/portal

Fleten, S., Maribu, K., Wangensteen, I., 2007. Optimal investment strategies in decentralized renewable power generation under uncertainty. Energy 32 (5), 803-815.

Geman, H., 2007. Mean reversion versus random walk in oil and natural gas prices. Advances in Mathematical Finance, 219-228.

Gotham, D., Muthuraman, K., Preckel, P., Rardin, R., Ruangpattana, S., 2009. A load factor based mean-variance analysis for fuel diversification. Energy Economics 31, 249-256.

IEA, 2010. World energy outlook. International Energy Agency, OECD.

Jansen, J., Beurskens, L., Tilburg, X., 2006. Application of portfolio analysis to the Dutch generating mix. Reference case and two renewables cases. Report c-05-100, ECN.

URL http://www.ecn.nl/publications/PdfFetch.aspx?nr=ECN-C--05-100

Konstantin, P., 2009. Praxisbuch Energiewirtschaft: Energieumwandlung, -transport und -beschaffung im liberalisierten Markt. Springer.

Mohammadi, H., 2009. Electricity prices and fuel costs: Long-run relations and short-run dynamics. Energy Economics 31, 503-509.

Pindyck, R., 1999. The long-run evolution of energy prices. The Energy Journal 20 (2), 1-27.

Pindyck, R., 2001. The dynamics of commodity spot and futures markets: a primer. Energy Journal 22 (3), 1-30.

Schwartz, E., 1997. The stochastic behavior of commodity prices: Implications for valuation and hedging. The Journal of Finance 52 (3), 923-973.

Schwartz, E., Smith, J., 2000. Short-term variations and long-term dynamics in commodity prices. Management Science 46 (7), 893-911.

StaBu, 2010. Genesis-online database, time series 61411-0006. Statistisches Bundesamt - German Federal Statistical Office.

URL https://www-genesis.destatis.de

Sunderkötter, M., Weber, C., 2009. Valuing fuel diversification in optimal investment policies for electricity generation portfolios. 
EWL Working paper 03/2009.

URL http://ideas.repec.org/p/dui/wpaper/0904.html

Trautmann, S., 2006. Investitionen - Bewertung, Auswahl und Risikomanagement. Springer.

Weber, C., 2005. Uncertainty in the Electric Power Industry: Methods and Models for Decision Support. Springer. 